\title{
Syntheses of functionalised $\beta$-amino acids through ring-opening/cross metathesis
}

\author{
PhD Thesis
}

\section{Márton Kardos}

Supervisors

Prof. Dr. Ferenc Fülöp

Prof. Dr. Loránd Kiss

Institute of Pharmaceutical Chemistry, University of Szeged

Szeged, Hungary 


\section{CONTENTS}

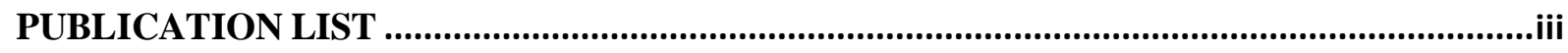

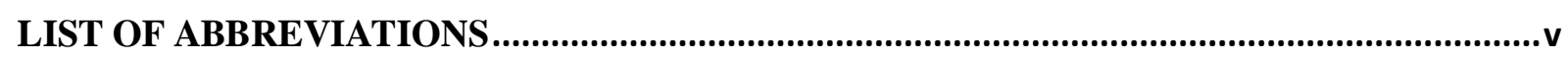

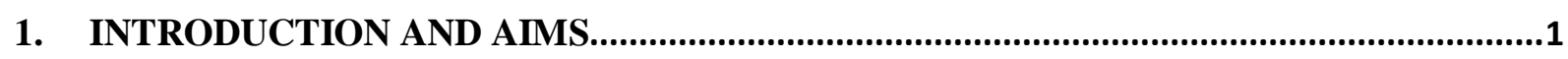

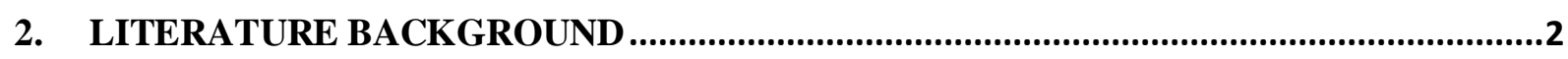

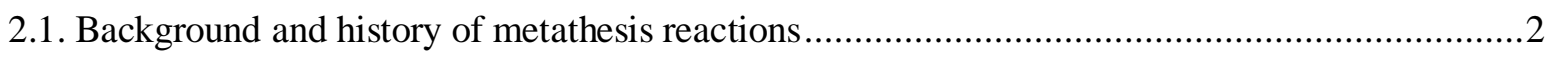

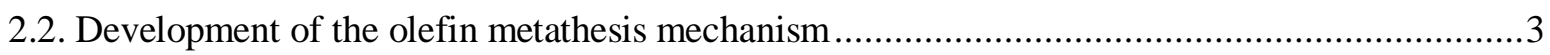

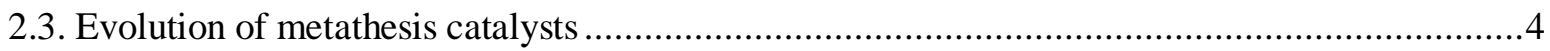

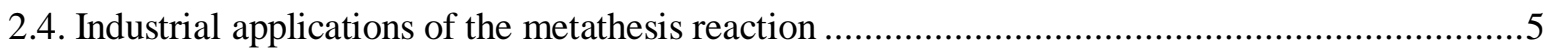

2.5. Application of metathesis for the synthesis of natural and bioactive products ..........................6

2.6. General rules for cyclisation through metathesis reaction......................................................

2.7. Synthesis and transformations of cyclic $\beta$-amino acids through metathesis reaction .................7

2.8. Synthesis of 5 - and 6 -membered cyclic $\beta$-amino acids by ring-closing metathesis (RCM) ........8

2.9. Synthesis of 5-and 6-membered cyclic $\beta$-amino acids by cross metathesis (CM) ..................13

2.10. Synthesis of $\beta$-amino acids with larger ring system by ring-closing metathesis (RCM) .........15

2.11. Synthesis of $\beta$-amino acids with condensed ring systems by ring-rearrangement metathesis

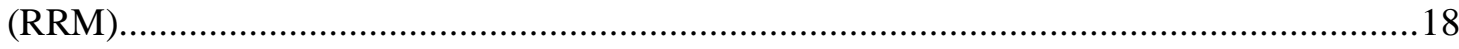

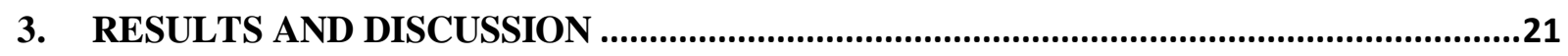

3.1. Stereocontrolled one-step synthesis of difunctionalised cispentacin and transpentacin derivatives through ring-opening metathesis (ROM) of norbornene $\beta$-amino acids ..............21

3.2. Stereocontrolled one-step synthesis of difunctionalised azetidinones and $\beta$-amino acid derivatives from condensed ring $\beta$-lactams by ring-opening metathesis (ROM) ...................26

3.3. Carbon-carbon double bond functionalisation of $\beta$-amino acid derivatives and $\beta$-lactams with $\alpha, \beta$-unsaturated carbonyl compounds through cross metathesis (CM).....

3.4. Syntheses of functionalised $\beta$-amino acid derivatives and $\beta$-lactams through chemoselective cross metathesis $(\mathrm{CM})$

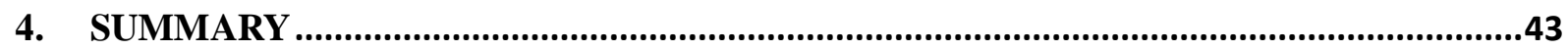

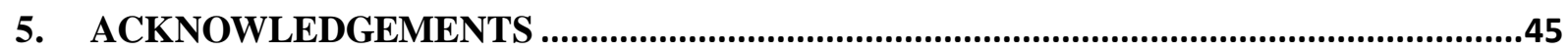

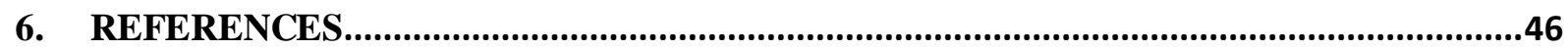

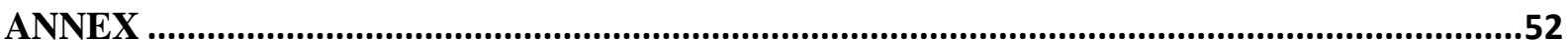




\section{PUBLICATION LIST}

\section{Papers related to the thesis}

I. Loránd Kiss, Márton Kardos, Enikő Forró, Ferenc Fülöp

Stereocontrolled one-step synthesis of difunctionalised cispentacin derivatives through ring-opening metathesis of norbornene $\beta$-amino acids Eur. J. Org. Chem. 2015, 1283.

IF: 3.068*

II. Márton Kardos, Loránd Kiss, Ferenc Fülöp

Stereocontrolled synthesis of difunctionalized azetidinones and $\beta^{2,3}$-amino acid derivatives from cyclodienes by ring-opening and cross-metathesis reactions Asian. J. Org. Chem. 2015, 4, 1155.

IF: $3.275 *$

III. Márton Kardos, Loránd Kiss, Matti Haukka, Santos Fustero, Ferenc Fülöp Olefin-bond chemodifferentiation through cross-metathesis reactions: a stereocontrolled approach to functionalized $\beta^{2,3}$-amino acid derivatives Eur. J. Org. Chem. 2017, 1894.

IF: 3.068*

*2015 impact factors 


\section{Conference lectures}

I. Kardos Márton, Kiss Loránd, Fülöp Ferenc

Telítetlen ciklusos $\beta$-aminosavak átalakításai metatézis reakcióval MTA Heterociklusos és Elemorganikus Kémiai Munkabizottság ülése

Balatonszemes, Hungary, 21-23, May, 2014, oral presentation

\section{Kardos Márton}

Funkcionalizált ciszpentacin származékok sztereokontrollált szintézise

biciklusos $\beta$-aminosavakból gyürünyitó metatézissel

XXXVII. Kémiai Elöadói Napok

Szeged, Hungary, 3-5, November, 2014, oral presentation

III. Kiss Loránd, Kardos Márton, Forró Enikő, Fülöp Ferenc

Funkcionalizált ciszpentacin származékok sztereokontrollált szintézise

biciklusos $\beta$-aminosavakból gyürünyitó metatézissel

XX. Nemzetközi Vegyészkonferencia

Cluj Napoca, Romania, 6-9, November, 2014, poster presentation

\section{Kardos Márton}

Funkcionalizált $\beta^{2,3}$-aminosav származékok sztereokontrollált szintézisei gyürünyitó/keresztmetatézissel

A Szegedi Ifjú Szerves Kémikusok Támogatásáért Alapítvány 14. Tudományos Elöadóülése

Szeged, Hungary, 29, April, 2015, oral presentation

V. Kardos Márton, Kiss Loránd, Fülöp Ferenc

Funkcionalizált $\beta^{2,3}$-aminosav származékok sztereokontrollált szintézisei biciklusos $\beta$-laktámok gyürünyitó/keresztmetatézisével MTA Heterociklusos és Elemorganikus Kémiai Munkabizottság ülése Balatonszemes, Hungary, 27-29, May, 2015, oral presentation

VI. Kardos Márton, Kiss Loránd, Fülöp Ferenc

Kemoszelektív keresztmetatézis $\beta$-aminosavszármazékokon MTA Heterociklusos és Elemorganikus Kémiai Munkabizottság ülése Balatonszemes, Hungary, 18-20, May, 2016, oral presentation 


\section{LIST OF ABBREVIATIONS}

ADMET

$\mathrm{CM}$

CSI

d.e.

DIEA

DMAP

DMF

d.r.

EDCI

e.r.

EYM

G1

$\mathrm{G} 2$

HG1

HG2

LDA

$\operatorname{LiN}(\mathrm{TMS})_{2}$

$m$-CPBA

PMP

$\mathrm{RCM}$

ROM

ROMP

RRM

SHOP

TEA

THF

$\mathrm{TsCl}$ acyclic diene metathesis polymerisation

cross metathesis

$\mathrm{N}$-chlorosulfonyl isocyanate

diastereomeric excess

$\mathrm{N}, \mathrm{N}$-diisopropylethylamine

4-dimethylaminopyridin

dimethylformamide

diastereomeric ratio

1-ethyl-3-(3-dimethylaminopropyl)carbodiimide

enantiomeric ratio

ene-yne metathesis

Grubbs Catalyst $1^{\text {st }}$ Generation

Grubbs Catalyst $2^{\text {nd }}$ Generation

Hoveyda-Grubbs Catalyst $1^{\text {st }}$ Generation

Hoveyda-Grubbs Catalyst $2^{\text {nd }}$ Generation

lithium diisopropylamide

lithium bis(trimethylsilyl)amide

meta-chloroperoxybenzoic acid

para-methoxyphenyl

ring-closing metathesis

ring-opening metathesis

ring-opening metathesis polymerisation

ring-rearrangement metathesis

shell higher olefin process

triethylamine

tetrahydrofuran

4-toluenesulfonyl chloride 


\section{INTRODUCTION AND AIMS}

As a consequence of their high biological relevance, $\beta$-amino acids have gained an important role in medicinal and organic chemistry in the last twenty years. These structures are present in many natural compounds either in free form or as part of more complex molecules. Certain representatives such as the 5-membered carbocyclic cispentacin (isolated from the culture broth of Bacillus cereus) or icofungipen possess strong antifungal activities. Moreover, new generation peptides built from $\beta$-amino acids show well-ordered secondary structures and exhibit stability against proteases or peptidases; therefore, they are important molecules for medicinal chemistry. ${ }^{1-16}$ Since the early 1990 s thanks to the effective development and commercial availability of well-defined Ru-based catalysts, olefin metathesis reactions have revolutionised the synthetic thinking. Metathesis, consequently, has become a powerful tool for the creation of one or more $\mathrm{C}-\mathrm{C}$ double bonds. With this new method in hand, many natural and biologically active compounds have been prepared, which were previously challenging or impossible to synthesize. Besides their utilisation at the laboratory scale, olefin metathesis has gained industrial applications as well. ${ }^{17-39}$

In the Institute of Pharmaceutical Chemistry at the University of Szeged, a number of synthetic methods were developed to access highly-functionalised cyclic $\beta$-amino acids. ${ }^{40-63}$ Although the synthetic approaches presented in these studies cover a broad spectrum of chemical transformations including selective techniques, olefin metathesis reactions were not utilised. It has been realised, however, that the numerous types of metathesis transformations [ring-closing metathesis (RCM), ring-opening metathesis (ROM), ring-opening metathesis polymerisation (ROMP), acyclic diene metathesis polymerisation (ADMET), ene-yne metathesis $(\mathrm{EYM})$, and cross metathesis $(\mathrm{CM})$ ] provide versatile protocols for the convenient preparation of diversely substituted $\beta$-amino acids and $\beta$-lactams.

The present $\mathrm{PhD}$ work focuses on the development of stereocontrolled synthetic methods for the preparation of novel alkenyl-functionalised $\beta$-amino acids and $\beta$-lactams through ring-opening metathesis reaction and on their subsequent functionalisation via cross metathesis taking place in highly selective manner in some cases. The ring-opening protocol is based on the high ring strain of the starting bi- or tricyclic compounds, which works as a driving force during stereocontrolled transformations. The products sometimes were prepared in optically pure form through enzymatic kinetic resolution of racemic compounds with azetidinone framework. 


\section{LITERATURE BACKGROUND}

\subsection{Background and history of metathesis reactions}

Over the last 50 years, olefin metathesis has revolutionised synthetic organic chemistry and became a powerful tool to create one or more $\mathrm{C}-\mathrm{C}$ double bonds under mild conditions with good functional group tolerance. This is a catalytic process in which redistribution of $\mathrm{C}-\mathrm{C}$ double bonds occur mediated by a transition metal-carbene complex. ${ }^{64}$ The word metathesis comes from the Greek $\mu \epsilon \tau \alpha \theta \epsilon \sigma \zeta \zeta$ meaning transposition and has been used for this kind of reaction since 1967. ${ }^{65-66}$ Metathesis reactions have gone through a long and difficult path to reach the present versatile form and they are not only new elements of the synthetic toolbar of organic chemistry, but changed the way chemists think about synthetic problems. ${ }^{67-75}$

The metathesis story started from three seemingly unrelated observations. In the late 1950s researchers at multiple companies reported the transformation of propylene to ethylene and 2-butenes in the presence of molybdenum compounds. It is interesting that in 1931 Schenider and Fröhlich reported a very similar transformation when they heated propylene at $752{ }^{\circ} \mathrm{C}$ without any catalyst, but their publication was ignored for a long time..$^{76-80}$ The next discovery was made in 1960 by a group at DuPont. They submitted norbornene to polymerisation resulting in a highly unsaturated polymer chain instead of the expected saturated one. ${ }^{81}$ Finally, in 1964, during the attempted polymerisation of cyclopentene with tungsten and molybdenum halide catalysts, Natta et al. reported the unexpected formation of an unsaturated polymer. ${ }^{82}$ These serendipities served a good starting point for further research.

In early times poorly-defined homogeneous and heterogeneous catalytic systems were used. These were based on transition metal compounds with strong Lewis acidic nature. Most functional groups did not tolerate these acids, which had many other drawbacks as well such as sensitivity to moisture or air. For these reasons in this time period the applicability of metathesis reaction was strongly limited. ${ }^{67}$ The breakthrough came when Grubbs published the first well-defined Ru-based catalyst, which successfully polymerised norbornene and exhibited high stability against oxygen and water. ${ }^{83}$ The real success for olefin metathesis comes from the wide range of transformations (RCM, ROMP, CM, ROM, EYM, and ADMET), which are feasible through this catalytic process (Fig. 1). A further useful property is that in most of the practically important cases the only side-product is a volatile olefin, which significantly facilitates purification. However, it should be taken into consideration that during metathesis reactions every starting material and possible product are in equilibrium with each other. 


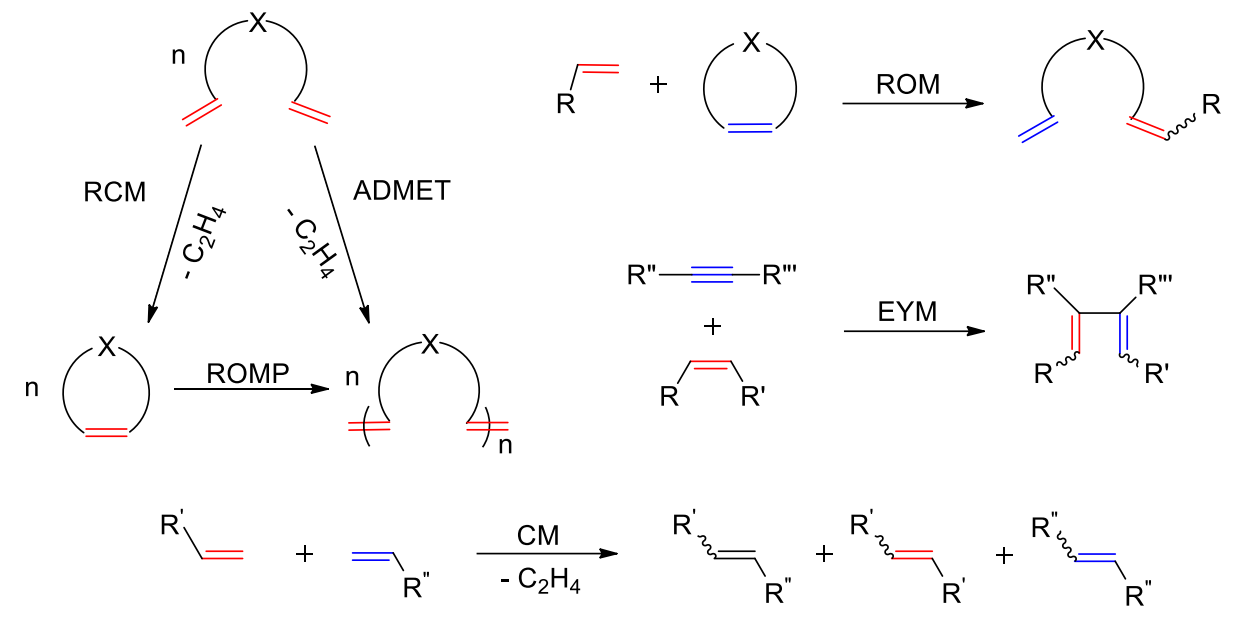

Figure 1.

\subsection{Development of the olefin metathesis mechanism}

Since the late 1950s researchers have been highly interested in olefin metathesis reactions because it seemed to be something mysterious, because numerous observations could not be explained on the basis of common knowledge at that time. The chemical community had to wait until 1967 for the first mechanistic proposal, which came from Bradshaw and was updated by Calderon in the following year (Fig. 2) ${ }^{84-86} \mathrm{~A}$ four-centred pathway was assumed in which the interchange of the $\mathrm{C}-\mathrm{C}$ double bonds takes place through a cyclobutane-metal complex intermediate ("pair-wise" mechanism). In 1972 Robert H. Grubbs, who had the primary role in the development of metathesis catalysts, also proposed a mechanism where the formation of the new olefin proceeds via a metallacyclopentane intermediate. ${ }^{87}$ However, at that time the mechanism accepted today was already known published in 1971 by Yves Chauvin and his student Jean-Louis Hérisson. ${ }^{64}$ Their theory, which is in agreement with all of experimental results, assumed the viability of a metal alkylidene (or metal carbene) complex. ${ }^{88-97}$ In the first step it reacts with the olefin in a $[2+2]$ cycloaddition (A reaction) to form the corresponding metallacyclobutane B. Note that [2+2] cycloadditions of alkenes are not allowed thermally, because of the symmetry rules. ${ }^{98}$ In the following cycloreversion step, the formation of both the new $\mathrm{C}-\mathrm{C}$ double bond and the new carbene complex occur by the redistribution of electrons (see $\mathbf{C})$. The new alkylidene complex $\mathbf{D}$ can start a new catalytic cycle again with an olefin. Every step of the mechanistic pathway is reversible, resulting in an equilibrium mixture. In 2005, when the Chemistry Nobel Prize was announced, the metathesis reaction was compared in an animation to dance, where the participants (olefins) can exchange their partner only if they make a dance with the master of ceremony (catalyst) before. ${ }^{99}$ 


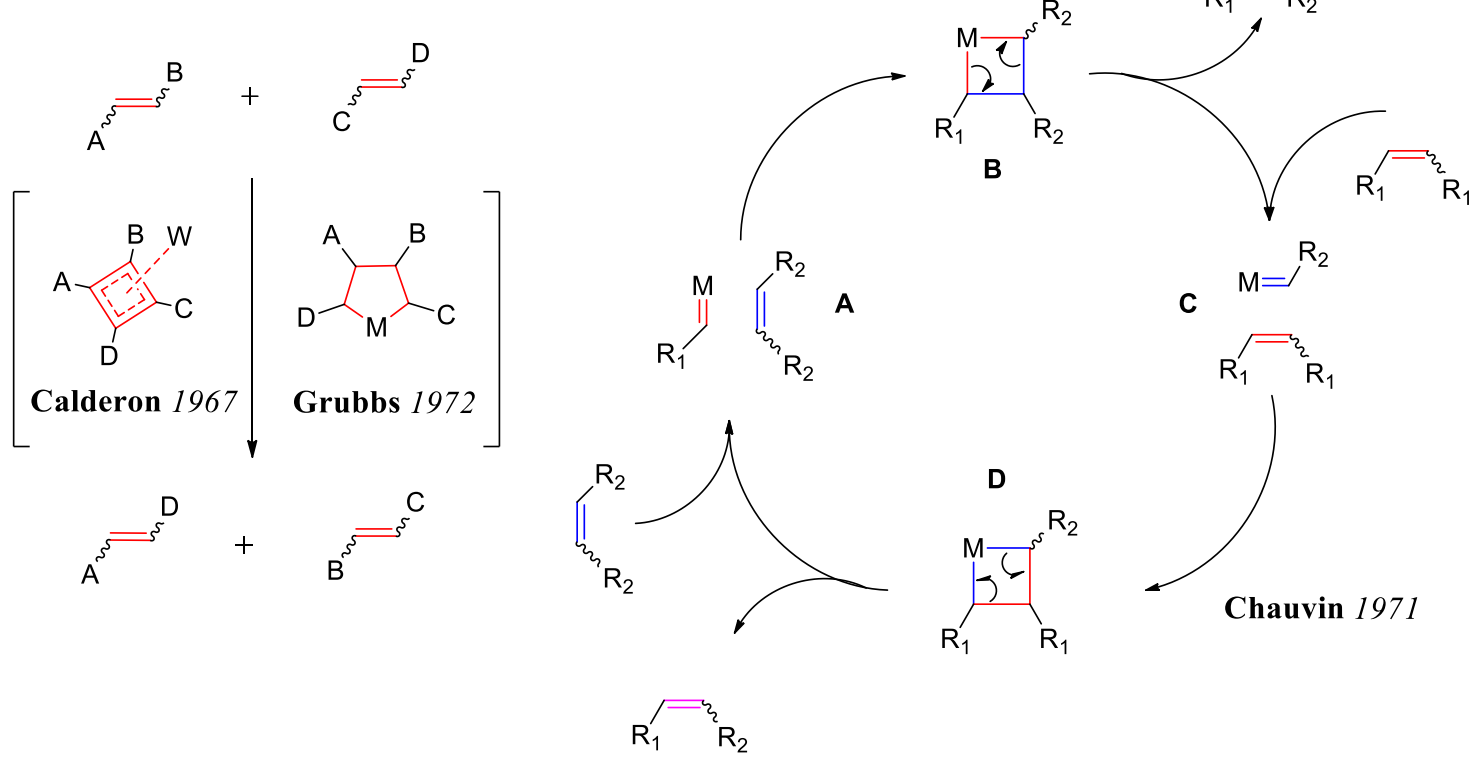

Figure 2.

\subsection{Evolution of metathesis catalysts}

As it was mentioned previously, ill-defined early catalytic systems had many drawbacks which, together with the need to understand the mechanism better, made catalyst development necessary ${ }^{67}$ In general, metathesis catalysts today can be divided into two categories based on the central metal. Schrock catalysts possess Mo or $\mathrm{W}$ in the coordination centre, while the Grubbs catalysts have Ru. This section intends to deal only with the evolution of Grubbs catalysts since during our research work Ru-based catalysts were exclusively used. Thanks to easy handling and good functional group tolerance, Grubbs catalysts have gained considerable attention among organic chemists as useful tools to create one or more $\mathrm{C}-\mathrm{C}$ double bonds. Their commercial availability enabled widespread utilisation, greatly contributing to the success of metathesis reactions. ${ }^{100-104}$

The first well-defined Ru catalyst for olefin metathesis was published in 1992 by Robert H. Grubbs (Fig. 3). ${ }^{83}$ After some modification of the ligands, Grubbs and co-workers reported the next well-defined catalyst three years later what is now known as the first generation Grubbs catalyst. ${ }^{105}$ These two communications have been real milestones in the metathesis history since from this time on, metathesis reactions do not need rigorous exclusion of air and moisture, making them available for practically everyone. In 1999 after an intensive study of ligand effect in metathesis reactions, a second generation catalyst was reported by Grubbs and co-workers. 
They have introduced a mixed catalyst where one phosphine of the parent complex was replaced by an aryl-substituted $N$-heterocyclic carbene ligand. ${ }^{106}$ While these catalysts possess superior activity and significantly expanded the scope of metathesis reactions, they still retained the beneficial properties of the first generation molecule.

Another significant episode during the time of catalyst development was the introduction of chelating benzylidene ligands by Hoveyda in 1999. ${ }^{107}$ This idea has opened numerous new possibilities to the fine tuning of the catalysts through the modification of the stability of the $\mathrm{Ru}-\mathrm{O}$ bond. These studies clearly showed that steric crowding around the chelating oxygen and an electron-withdrawing group on the benzylidene ring lead to the weakening of the $\mathrm{OiP} \rightarrow \mathrm{Ru}$ bond resulting in faster initiation. ${ }^{108-112}$ It is worth mentioning that the detailed mechanistic pathway of the Hoveyda catalysts was a long-standing question although the release-return mechanism (boomerang effect) became a widely accepted theory. ${ }^{113}$

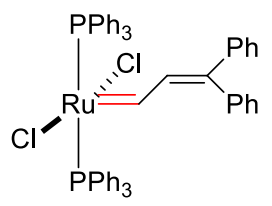

The first well-def. cat. (1992)

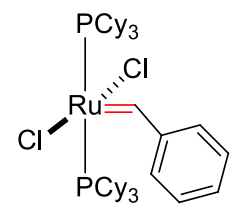

Grubbs $1^{\text {st }}$ (1995)

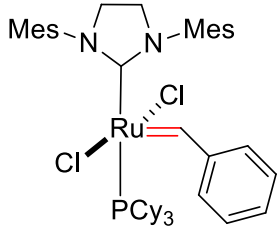

Grubbs $2^{\text {nd }}$ (1999)

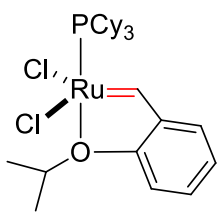

Hoveyda-Grubbs $1^{\text {st }}$

(1999)

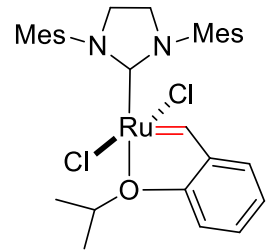

Hoveyda-Grubbs $2^{\text {nd }}$

(2000)

Figure 3.

\subsection{Industrial applications of the metathesis reaction}

Beside of research laboratories, olefin metathesis reactions appeared in different fields of industrial chemistry including the production of petrochemicals, oleochemicals, and polymers. ${ }^{33-39}$ It is difficult to select, which should be discussed among industrial applications, because of the widespread exploitation of metathesis reactions. Nevertheless, the following processes cannot be ignored. The Phillips Triolefin Process was originally developed for the production of ethylene and 2-butene by the CM reaction of propylene. Taking advantage of the equilibrium nature of metathesis, today this reaction is utilised in the reverse direction because of the high commercial demand of propylene. ${ }^{34}$ Almost 50 years before, an elegant process was elaborated for the manufacture of primary $\mathrm{C}_{11}-\mathrm{C}_{15}$ fatty alcohols in the laboratories of Shell Development Company. The Shell Higher Olefin Process (SHOP) consists of four main parts (oligomerisation, linear hydroformylation, isomerisation, and metathesis). In the last step, olefin metathesis is responsible for the utilisation of "nonmarketable" olefins. ${ }^{114}$ 
Last but not least, olefin metathesis has a huge impact in the field of polymer chemistry. Several strained cyclic and bicyclic olefins have been polymerised by ROMP. One of the best-known industrial products is Telene ${ }^{\circledR}$ or Metton $^{\circledR}$ manufactured by the polymerisation of dicyclopentadiene. ${ }^{34}$

\subsection{Application of metathesis for the synthesis of natural and bioactive products}

Since the discovery of well-defined Ru-based catalysts, several naturally occurring and biologically active products were prepared where some metathesis reactions was utilised as a key step during the procedure. ${ }^{25-31}$ In this field Ring-Rearrangement Metathesis (RRM) is especially useful, because it enables the formation of highly diverse unsaturated ring systems in a tandem fashion. ${ }^{115-116}$ This reaction has many advantages over the usual stepwise approach, such as decreasing the number of steps, and minimizing the necessary reagent, solvent, and purification. These benefits were utilised in 1999 by Granja et al. when "taxosteroid" containing the same $[5,3,1]$ bicyclic ring system as taxol was synthesised via cascade dienyne metathesis reaction (Fig. 4) ${ }^{117}$ RCM was applied successfully during the synthesis of the potential cancer drug epothilone $A$ or the hepatitis C virus (HCV) protease inhibitor BILN 2061 to form the corresponding macrocycle. ${ }^{118-119}$ Chemoselectivity achievable by selecting the appropriate catalyst even in the case of highly-functionalised molecules is another great advantage of metathesis reactions. ${ }^{120}$

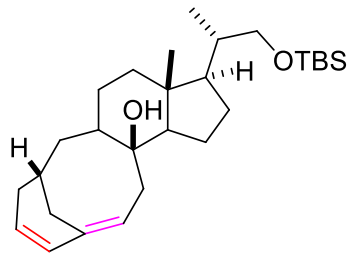

Taxosteroid

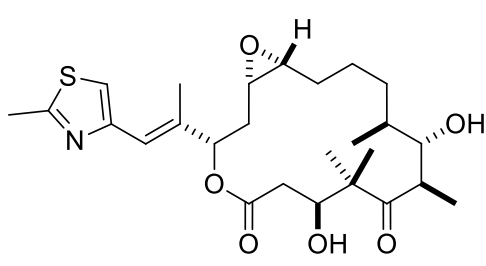

Epothilone $A$

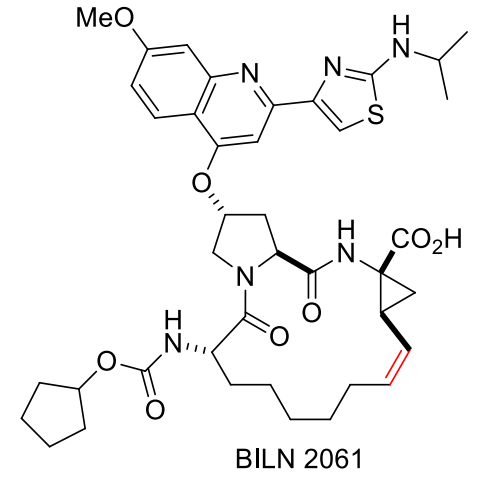

BILN 2061

Figure 4. 


\subsection{General rules for cyclisation through metathesis reaction}

Thanks to the well-defined Ru-based Grubbs catalysts, RCM reactions evolved into a very popular and widely used method for the construction of simple or more complex ring systems from the corresponding diene. The literature became enormous and excellent studies were published over the last two decades. A further feature that increases the value of this process is that not only carbocyclic compounds but also their heterocyclic counterparts can be prepared (Fig. 5). Basically, two factors determine the equilibrium and, therefore, the outcome of the RCM: entropy and enthalpy. Removal of the formed volatile ethylene, which can be accelerated with increasing the temperature, results in entropic gain, which facilitates both ring-closing and polymerisation of the diene. In order to prefer cyclisation over polymerisation, the use of low concentrations is a commonly applied technique. On the other hand, enthalpy also plays a crucial role during ring formation. Creation of thermodynamically stable 5- or 6-membered cyclic compounds is usually readily accomplished with RCM protocol. Problems, however, arise when larger or strained ring systems are desired. Finally, it is worthwhile to mention that selection of both the appropriate catalyst and its quantity are of pivotal importance. Unfortunately, a universally applicable catalyst, which outperforms all others in all cases, does not exist, and the amount of the catalyst used strongly influences the outcome of the reaction..$^{121-126}$

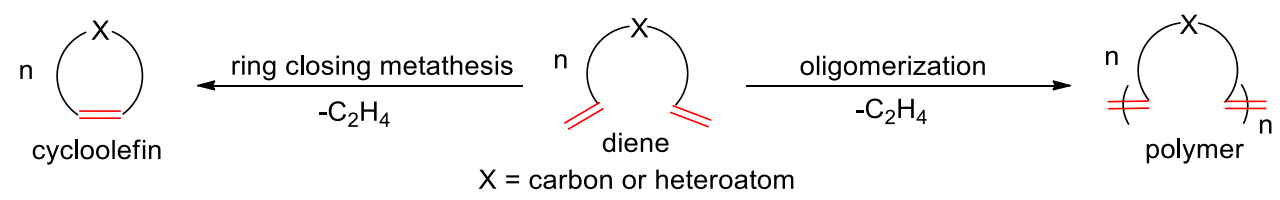

Figure 5.

\subsection{Synthesis and transformations of cyclic $\beta$-amino acids through metathesis reaction}

Over the last two decades, olefin metathesis reactions have gained widespread application towards the access of cyclic $\beta$-amino acid. Modern Ru-based catalysts greatly facilitated the proliferation of this method, since they are applicable on densely-functionalised molecules leading to the formation of complex products. ${ }^{67}$ Ring-closing metathesis is a frequently applied key step to construct cyclic frameworks with different sizes. ${ }^{124}$ The formed $\mathrm{C}-\mathrm{C}$ double bond affords great possibilities for further functionalisation such as epoxidation, dihydroxylation, or Diels-Alder reactions. This pathway requires furnishing the acyclic compounds with alkenyl groups to enable cyclisation. A less common approach is application of cross metathesis to form 
appropriate acyclic precursors for subsequent cyclisation. In most cases, protocols developed for the construction of cyclic $\beta$-amino acids are suitable for the preparation of different ring sizes. Publications usually describe preparation of both 5- and 6-membered cycles using the same method; therefore, these syntheses were summarised separately from the construction of larger or condensed rings. Numerous research groups utilised metathesis reaction toward cyclic $\beta$-amino acids and some of the major developments are summarised below.

\subsection{Synthesis of 5- and 6-membered cyclic $\beta$-amino acids by ring-closing metathesis $(\mathrm{RCM})$}

Abell and Gardiner have elaborated an elegant process by RCM for the construction of 6-membered cyclic cis and trans $\beta^{2,3}$-amino esters substituted or unsubstituted at the $\alpha$-position (Scheme 1). ${ }^{127}$ First, acyclic $\beta$-amino ester $( \pm)$-1 (obtained from protected allyl-glycine) was transformed into diene $( \pm)-2$ by stereoselective allylation. This was followed by RCM in the presence of Grubbs $1^{\text {st }}$ generation catalyst (G1) smoothly affording trans cyclohexene $\beta^{2,3}$-amino acid derivative $( \pm)$-3 with cyclohexene skeleton. Saturated derivative $( \pm)-\mathbf{4}$ was easily accessible with the reduction of the $\mathrm{C}-\mathrm{C}$ double bond by hydrogenation on $\mathrm{Pd} / \mathrm{C}$.
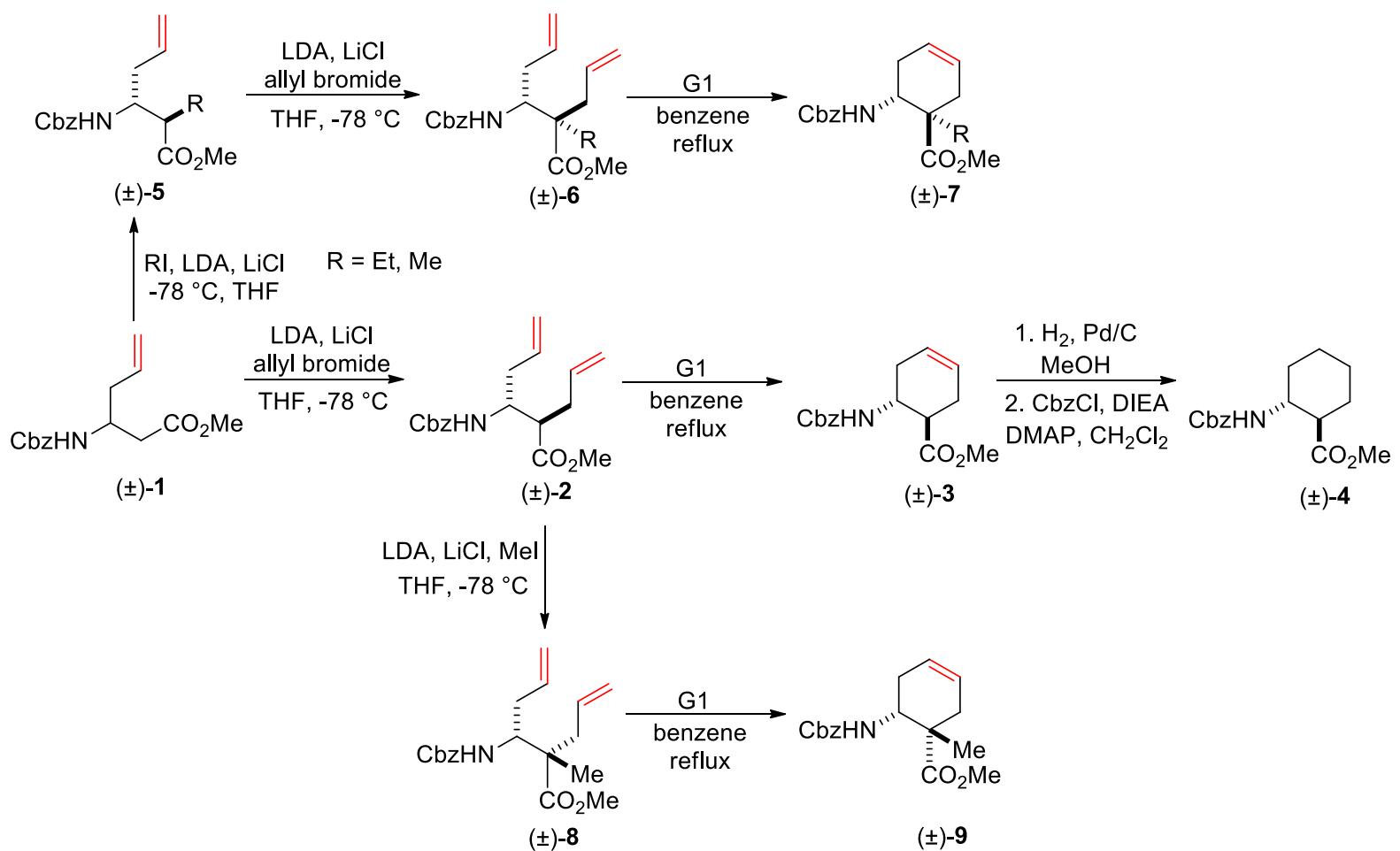

Scheme 1.

To extend the former approach, $\alpha$-substituted cyclic $\beta$-amino acids $( \pm)-7$ and $( \pm)$-9 were synthesised (Scheme 1). The relative stereochemistry of the carboxylic and the amino functions 
depended on the sequence of alkylation. When compound ( \pm )-1 was first reacted with alkyl iodide and then with allyl bromide, the cyclisation of resulting ( \pm )-6 afforded trans derivative $( \pm)-7$. However, if the alkylation sequence was changed (allylation followed by alkylation) compound ( \pm )-8 was formed, which can be cyclised to cis amino acid ( \pm )-9. Noteworthy that the use of optically pure $\mathbf{1}$ prepared using Evans chiral auxiliary group enables the synthesis of (-)-3 and (-)-4 in enantiopure form.

The same group published an extended version of the above method for the synthesis of cyclic $\beta^{2,3}$-amino acid derivatives substituted or unsubstituted at the $\alpha$-position (Scheme 2). ${ }^{128}$ This approach is suitable for the preparation of 5-, 6- and 7-membered ring systems. From readily accessible optically active $\alpha$-amino acid building blocks such as $(S)$-methionine or $(R)$-allyl glycine, enantiopure 5- or 6-membered cyclic $\beta^{2,3}$-amino acids can easily be prepared. The first step of the synthetic strategy towards 5-membered rings was an Arndt-Eistert carbon chain elongation of Cbz-protected $(S)$-methionine-10 to form the corresponding $\beta$-amino acid (-)-11, which was subsequently submitted to stereoselective allylation with allyl bromide and LDA in the presence of $\mathrm{LiCl}$. The other alkenyl group, necessary for the cyclisation [product (-)-13], was formed in the next step by oxidative elimination.

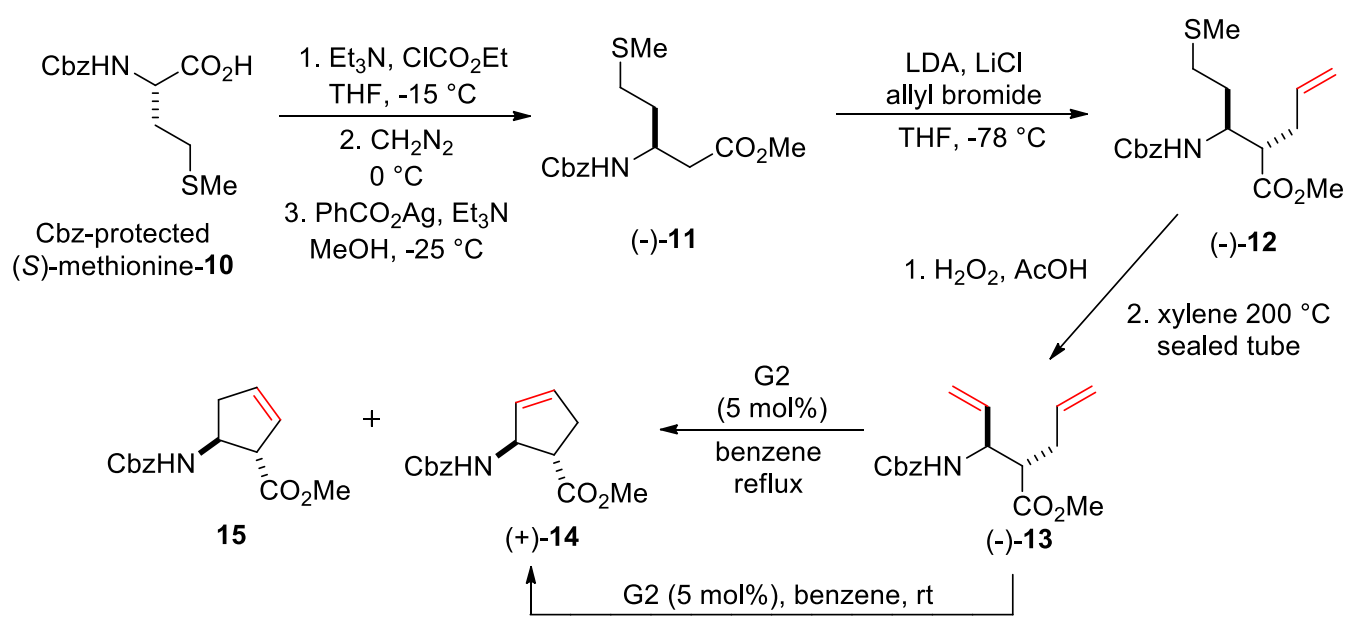

Scheme 2.

Compound (-)-13 was readily transformed to 5-membered cyclic $\beta^{2,3}$-amino acid in the presence of Grubbs $2^{\text {nd }}$ catalyst $(\mathrm{G} 2)$ with excellent yield. However, if the temperature was increased both the expected ring-closure product (+)-14 and its isomerised derivative $\mathbf{1 5}$ were formed in a 1.5:1 ratio. As it was mentioned above, this synthetic strategy is suitable for the preparation of $\alpha$-substituted cyclic derivatives. For this purpose, compound (-)-11 was stereoselectively alkylated by MeI, which gave the $\alpha$-methyl substituted ester as a single diastereomer (-)-16 (Scheme 3). 
Diastereoselective allylation of compound (-)-16 gave product (-)-17, which was transformed by oxidative elimination to acyclic $\beta$-amino acid derivatives (-)-18 with two alkenyl side-chains.

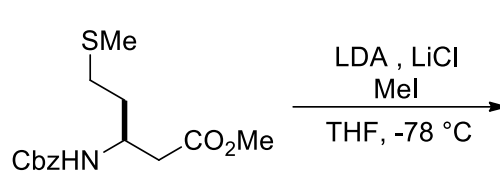

$(-)-11$<smiles>CCCNC(CCC)C(C)C(C)=O</smiles>

$(-)-16$

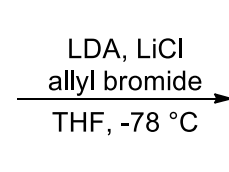

$(-)-17 \mathrm{CO}_{2} \mathrm{Me}$

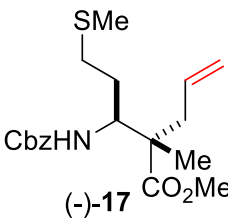

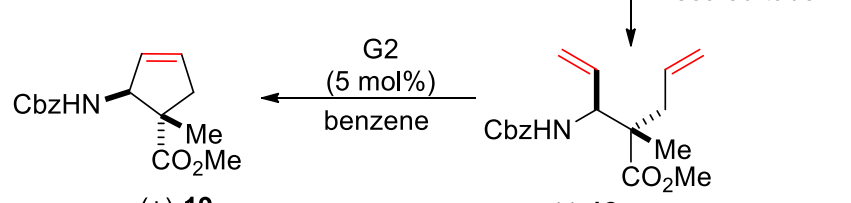

$(+)-19$

$(-)-18$

Scheme 3.

To avoid isomerisation experienced before, RCM of compound (-)-18 was executed at room temperature with $\mathrm{G} 2$ catalyst to afford unsaturated 5-membered $\alpha$-methyl substituted cyclic $\beta^{2,3}$-amino ester $(+)$-19. The authors also improved their method towards enantiopure 6-membered cyclic $\beta$-amino esters by finding an alternative route to prepare starting compound 1. Asymmetric synthesis was used to prepare Cbz-protected $(R)$-allyl glycine, which was submitted to Arndt-Eistert homologation resulting in optically pure (+)-1. Following the steps of Scheme 1 led to (-)-3 and (-)-4.

Chippindale and co-workers developed a versatile method enabling the stereoselective synthesis of 5 -membered cyclic $\beta^{2,3}$-and $\beta^{3}$-amino acids and 6 -membered cyclic $\beta^{3}$-amino acids. ${ }^{129}$ First, compounds (+)-22a,b were synthesised by conjugate addition of lithium (S)-allyl(1-phenylethyl)amide 20 to $\alpha, \beta$-unstaurated esters 21a,b with high d.e. (Scheme 4). RCM reaction of (+)-22a, $\mathbf{b}$ resulted in $N$-heterocyclic $\beta^{3}$-amino acid derivatives (-)-23a and (+)-23b, which were transformed into free amino acids (+)-24a,b.

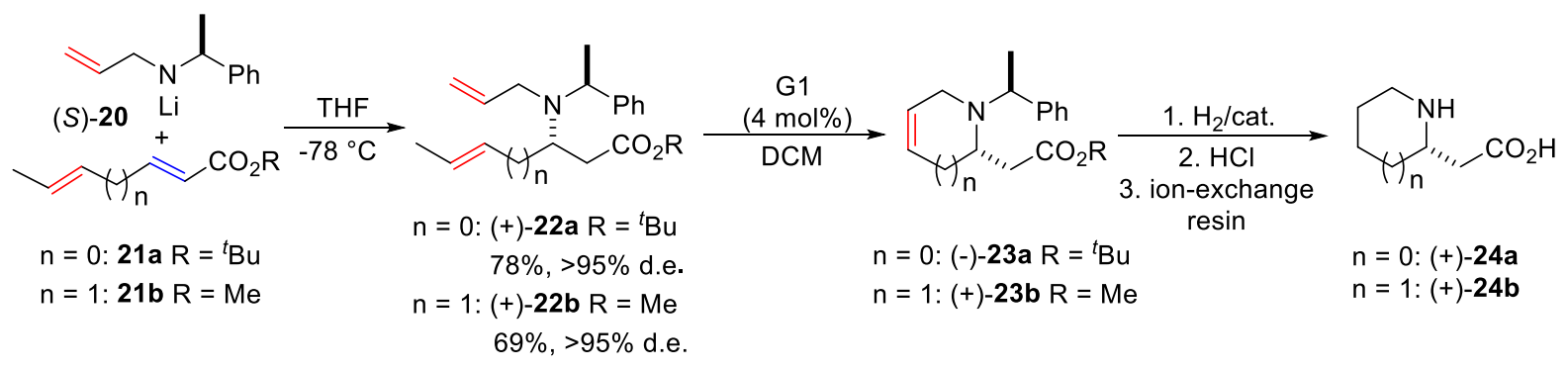

Scheme 4. 
In order to synthesize cyclic $\beta^{2,3}$-amino acids, allylation of intermediate (+)-22a was performed affording an inseparable mixture of anti and syn diastereomers 25 (Scheme 5). However, the related secondary amines obtained through $N$-deallylation of compound 25 with Wilkinson catalyst were separable by column chromatography and the major (anti) diastereomer (-)-26 was isolated with $>95 \%$ d.e. and in 77\% yield. Based on earlier experience, the amine function was transformed to carbamate in order to avoid chelate formation, which greatly reduces the activity of the Grubbs catalyst. Cyclisation of (-)-27 by RCM led to cyclic $\beta^{2,3}$-amino acid derivative (+)-28, which was subsequently deprotected to afford transpentacin (+)-29.

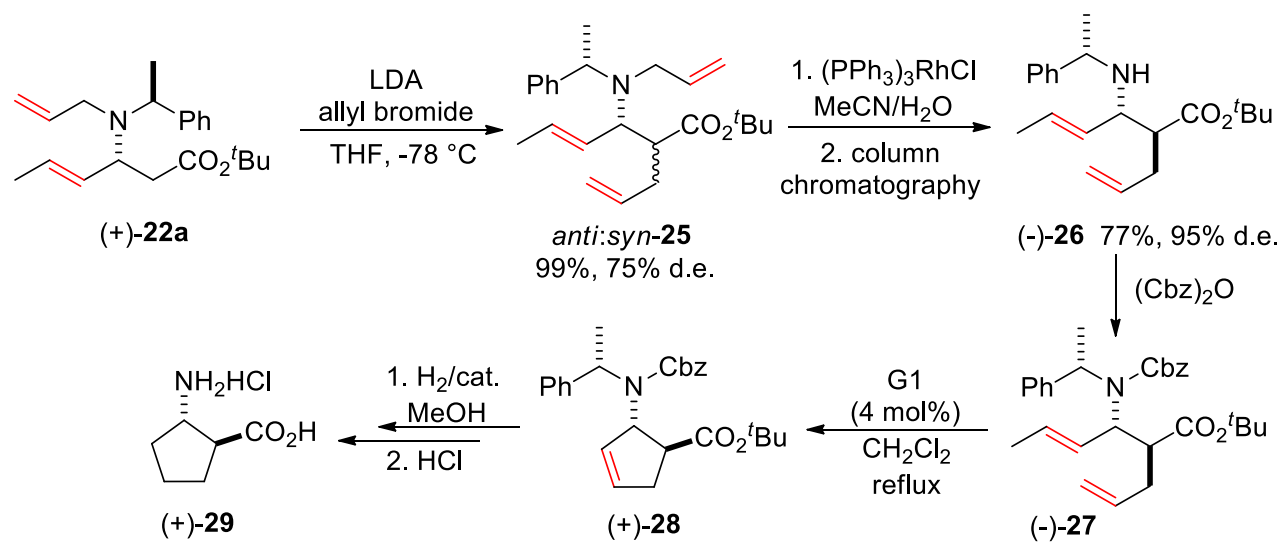

Scheme 5.

Davis and Theddu elaborated a general methodology for the asymmetric synthesis of cyclic cis- $\beta$-amino Weinreb amides, which are easily convertible in two steps to cyclic cis $\beta^{2,3}$-amino acid derivatives. ${ }^{130}$ As depicted in Scheme 6, optically pure acyclic dienes (+)-32a,b were synthesised with varied alkenyl chains by the conjugate addition of unsaturated prochiral Weinreb amide 31a,b (enolates generated by LDA) to chiral sulfinimine $(S)-(+)-30$ with high $>99: 1$ d.r. Oxidation of compounds (+)-32a,b at sulfur resulted in tosyl-protected compounds (-)-33a,b. RCM of compounds (+)-32a,b and (-)-33a,b with G2 catalyst gave the expected cyclic $\beta$-amino Weinreb amides (+)-34a,b and (+)-35a,b, respectively. The possibility of liberating the carboxyl group was studied in details in the case of 5-membered derivatives (+)-34a and (+)-35a. Both compounds decomposed during hydrolysis; however, in the case of Weinreb amide (+)-34a, saturation of the ring followed by hydrolysis smoothly afforded aminocarboxylic acid (-)-36. 


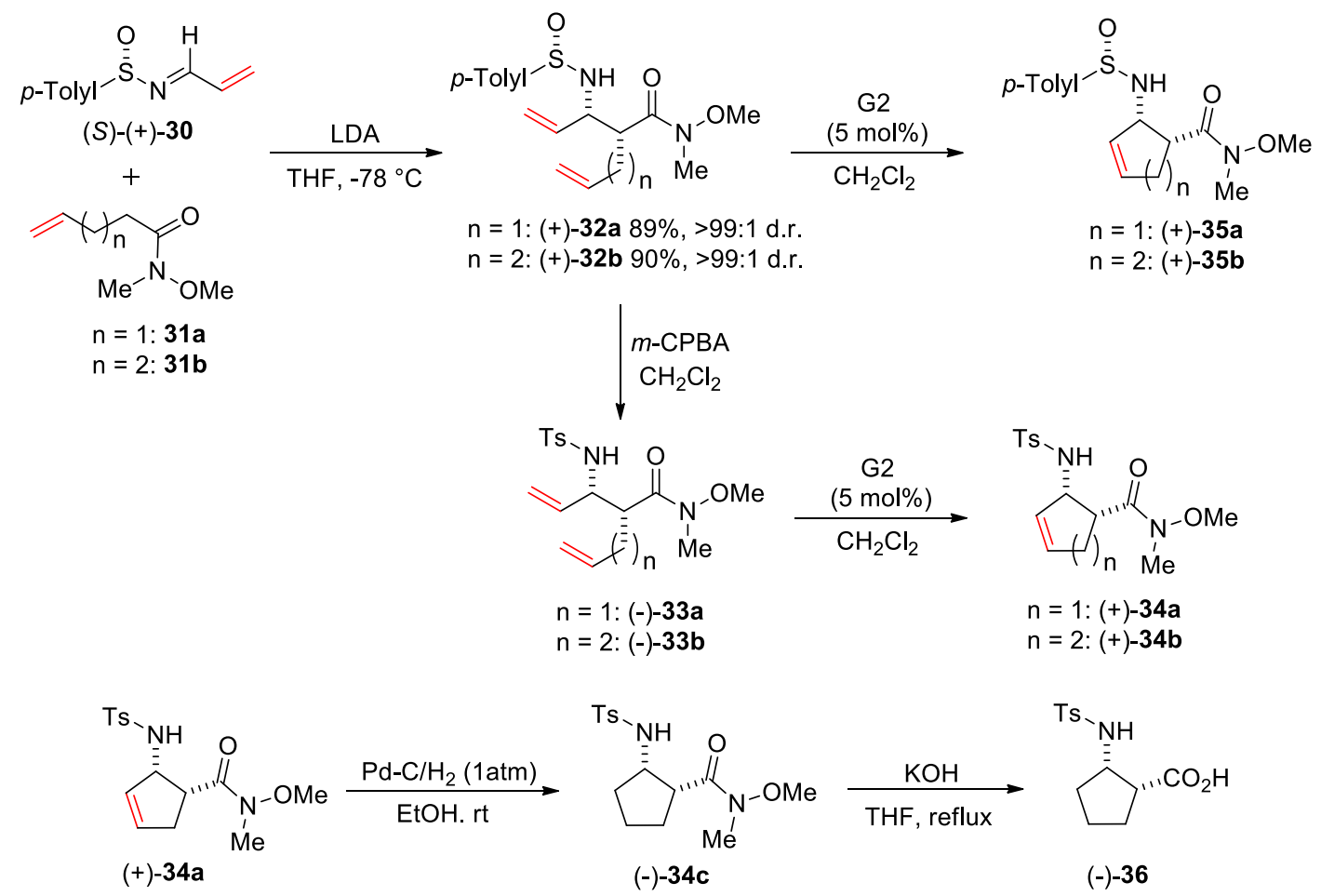

Scheme 6.

The strategy discussed above in detail is applicable for the asymmetric synthesis of 7-membered cyclic cis- $\beta$-amino Weinreb amides too. It is worth mentioning that in addition to $\beta$-amino acids, several valuable molecules are achievable via Weinreb amides, such as ketones, aldehydes, $\beta$-ketoesters, and $\beta$-ketophosponates.

A stereoselective route towards cyclic $\beta^{2,3}$-amino acids was developed by Perlmutter et al. in which the bonds formed by nucleophilic addition $\left(\mathrm{A}_{\mathrm{N}}\right)$ were transformed by ring closure (RC) (NARC protocol). ${ }^{131}$ This is a frequently applied method for the synthesis of hetero- and carbocyclic compounds. ${ }^{132}$ A key step of the procedure is the diastereoselective condensation between pyridyl thioesters 37a,b and optically pure imine $(R)-\mathbf{3 8}$ (Scheme 7). The reaction provided a mixture of diastereomers [(+)-39 is the major and $\mathbf{4 0}$ the minor product], which were readily separable by flash chromatography. Attempted ring closure of $(+)-39 a, b$ to form bicyclic $\beta$-lactams were unsuccessful becau se of the evolving ring strain. To avoid this synthetic problem the 4-membered heterocyclic rings were opened to get acyclic dienes $(+)-\mathbf{4 1 a}, \mathbf{b}$, which easily underwent RCM with G1 catalyst. Hydrogenation of the double bond then hydrolysis with $\mathrm{HCl}$ afforded optically pure 5- and 6-membered saturated cyclic $\beta$-amino acid hydrochlorides (+)-29 and (+)-43. 


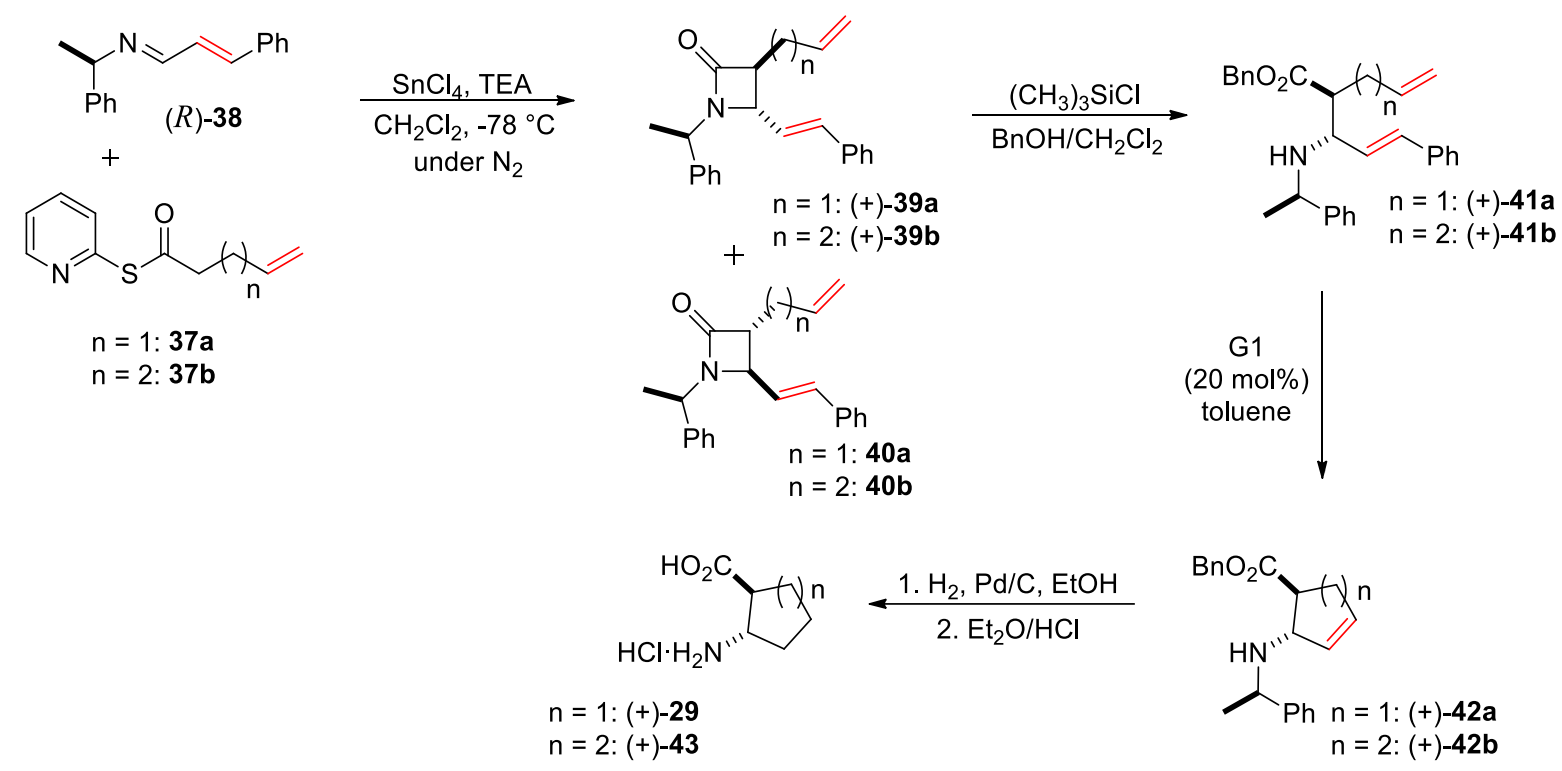

Scheme 7.

\subsection{Synthesis of 5- and 6-membered cyclic $\beta$-amino acids by cross metathesis $(\mathrm{CM})$}

While several open-chain dienes have been successfully transformed by RCM, only a few examples were reported where $\mathrm{CM}$ was utilised toward cyclic $\beta$-amino acids. In these cases, the role of metathesis was the preparation of appropriate acyclic precursors, which were cyclised by intramolecular addition or condensation. In one of these examples, Fustero and co-workers recently reported a diastereodivergent synthesis of enantiomerically pure fluorinated homoproline derivatives (cyclic $\beta^{3}$-amino acids) with three chiral centers. ${ }^{133}$ As shown in Scheme 8, deprotonation of sulfoxide $(S)-\mathbf{4 4}$ at the benzylic position led to the corresponding carbanion. Addition of the latter to fluorine-containing aldimine $\mathbf{4 5}$ yielded amine 46 with high diastereoselectivities. Cross metathesis performed with ethyl acrylate 47 in the presence of Hoveyda-Grubbs $2^{\text {nd }}$ catalyst (HG2) gave $\mathbf{4 8}$ as a mixture of $E / Z$ diastereomers. Cyclisation via intramolecular aza-Michael reaction can result in two epimers of $\mathbf{4 9}$ with relative syn or anti arrangement of the fluorinated alkyl group and the $\mathrm{CH}_{2} \mathrm{CO}_{2}$ Et moiety.

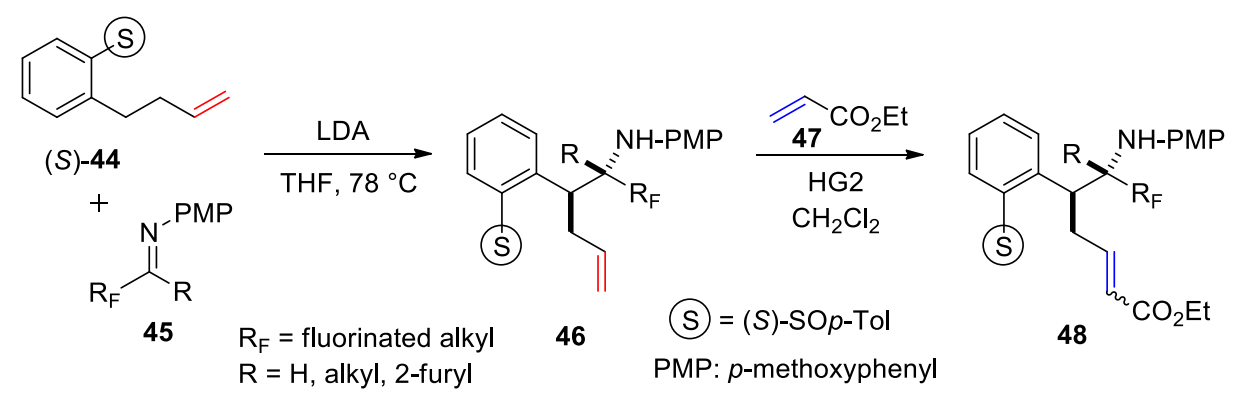

Scheme 8. 
Diastereoselectivity depended on the reaction conditions: base-promoted cyclisation gave the anti epimer as the main product, while treatment with Lewis acidic $\mathrm{BF}_{3} \cdot \mathrm{OEt}_{2}$ afforded mostly the syn epimer because of the formation of a chelate ring involving the ester carbonyl, the sulfoxide and the nitrogen atom (Scheme 9). Removal of the chiral auxiliary was achieved in the final step using Raney Ni catalyst in THF to give homoproline derivatives $\mathbf{5 0 .}$

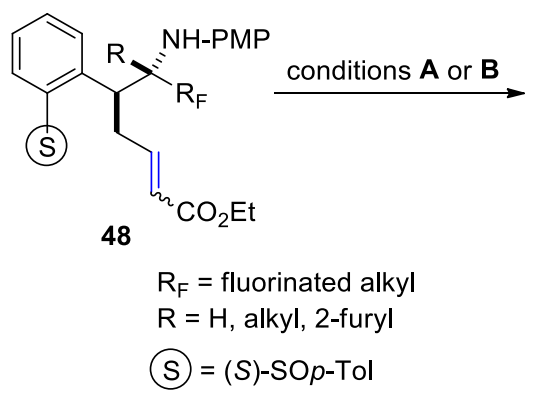

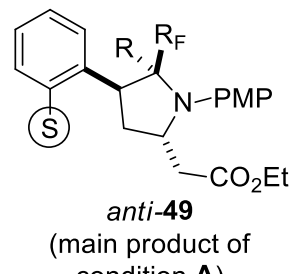

condition $\mathbf{A}$ )

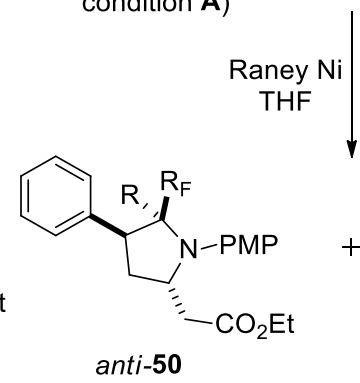

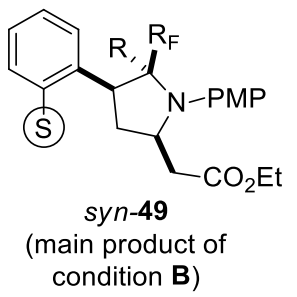

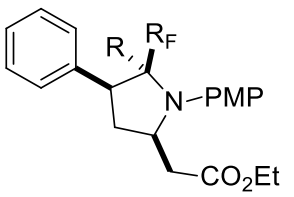

syn-50

Scheme 9.

The second example was also elaborated by the same research group. They successfully conducted racemic and asymmetric syntheses of difluorinated 5-, 6-, and 7-membered (see later) cis cyclic $\beta^{2,3}$-amino acids through CM (Scheme 10). ${ }^{134}$ In the first step, imidoyl chlorides 52a,b were prepared from the corresponding 2,2-difluorinated carboxylic acids 51a,b. Cross-coupling reaction between these imidoyl chlorides and ethyl acrylate 47 gave the expected acyclic compounds $\mathbf{5 3 a}, \mathbf{b}$. This is a remarkable achievement as the first reported case of using the $\mathrm{G} 2$ catalyst in the reaction of imidoyl chlorides.

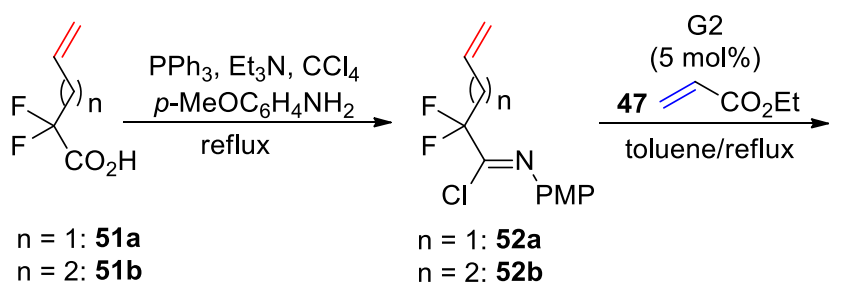

PMP: $p$-methoxyphenyl
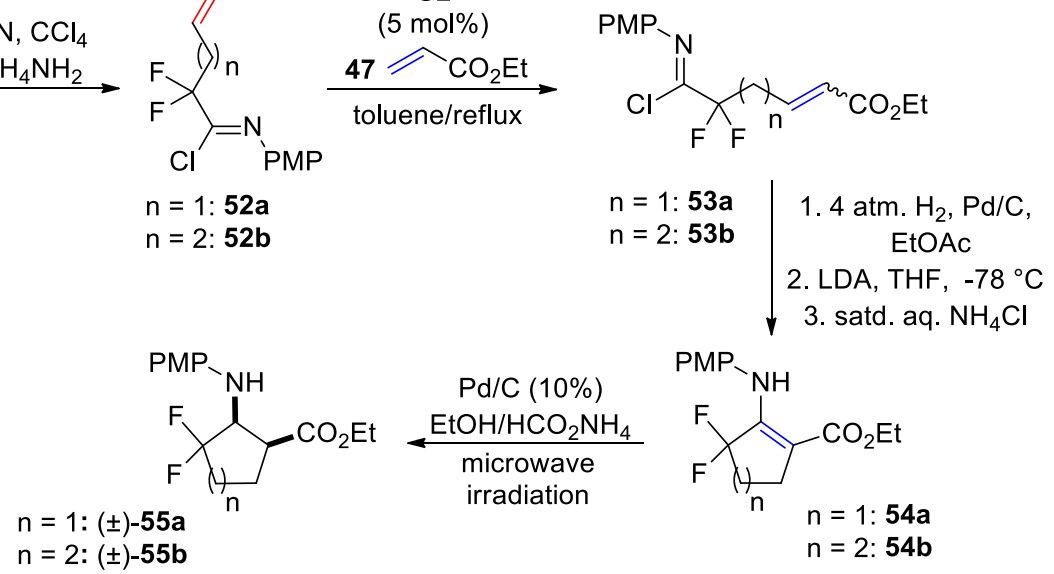

Scheme 10. 
Chemoselective hydrogenation of the $\mathrm{C}-\mathrm{C}$ double bond and subsequent Dieckmann condensation with LDA at $-78^{\circ} \mathrm{C}$ resulted in the formation of cyclic enamino compounds $\mathbf{5 4 a}, \mathbf{b}$. After several experiments, chemo- and stereoselective reduction was accomplished to furnish the final product $( \pm)$-55a,b with cis configuration. In the final step the method was extended to the preparation of enantiomerically pure compounds. For this purpose, $(+)-8$-phenylmenthol acrylate was coupled with imidoyl chloride 52a to afford the optically pure analogue of compound 53a, which was further transformed similarly to the racemic counterpart (53a).

\subsection{Synthesis of $\beta$-amino acids with larger ring system by ring-closing metathesis (RCM)}

Construction of larger rings has challenged organic chemists for a long time; however, several techniques are available in the synthetic toolbar today. ${ }^{135} \mathrm{RCM}$ opened up new possibilities for the syntheses of medium rings to macrocycles, because low substrate concentrations prefer ring closing over polymerisation even in the case of these challenging ring systems. ${ }^{124}$ Among several compounds, $\beta$-amino acid derivatives were also prepared in this manner. Of the methods described in Sections 2.8 and 2.9, four strategies were applicable for the construction of larger ring systems.

The method developed by Gardiner and co-workers, where 5- and 6-membered cyclic $\beta^{2,3}$-amino esters were prepared from easily accessible $\alpha$-amino acids building blocks, was extended to create 7-membered rings (Scheme 11). ${ }^{128}$ Acyclic precursor (-)-57, synthesised from $(S)$-serine-56 in multiple steps similar to (-)-13, was cyclised by G2 catalyst then hydrogenated on Pd-carbon to give the target compound (+)-59.

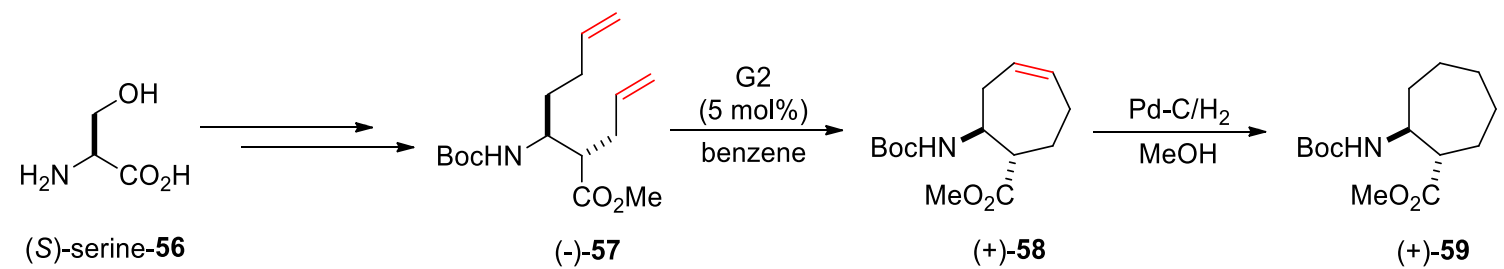

Scheme 11.

Diastereoselective conjugate addition of lithium $(S)-N$-allyl- $N$ - $\alpha$-methylbenzylamide 20 to $\alpha, \beta$-unsaturated ester 21 a developed by Chippindale and co-workers is also applicable for the preparation of larger ring. ${ }^{129}$ As depicted in Scheme 12 compound (-)-60, which was synthesised in a similar fashion to (-)-27 (see section 2.8 for the detailed pathway), was treated with G1 catalyst to afford 7-membered unsaturated cyclic molecule (+)-61. 


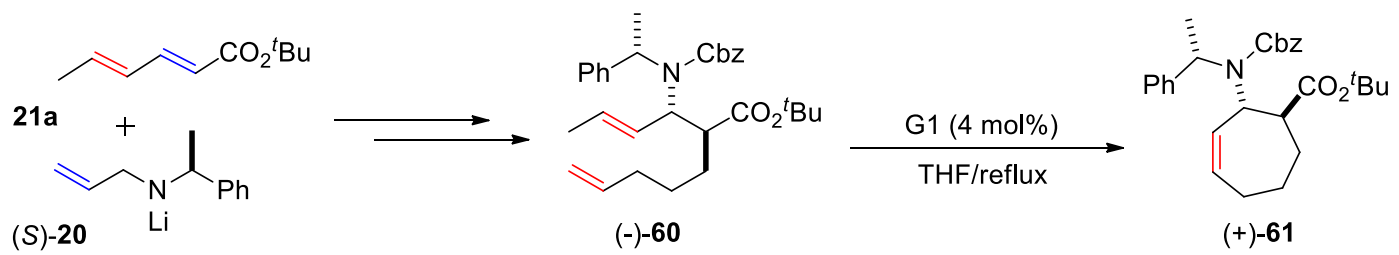

Scheme 12 .

Davis and Theddu successfully applied their approach (detailed in Scheme 6) for the preparation of a 7-membered ring by subjecting prochiral Weinreb amide 62 to conjugate addition with acrolein-derived sulfinimine $(S)-(+)-30 .{ }^{130}$ Precursor $(+)-63$ thus formed was easily cyclised with G2 catalyst to 7 -membered cyclic $\beta$-amino acid derivative (+)-64 (Scheme 13).

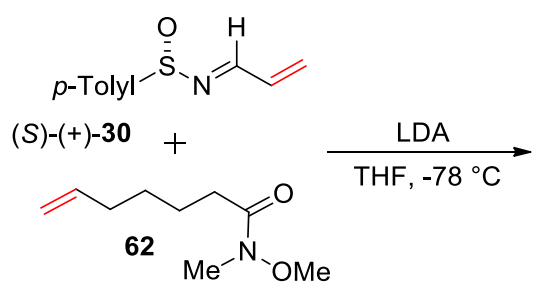

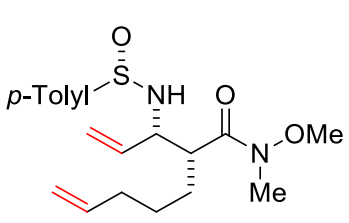

$(+)-6390 \%,>99: 1$ d.r.

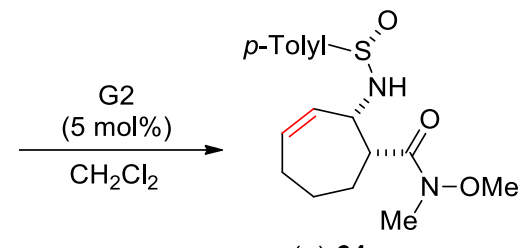

$(+)-64$

Scheme 13.

The method developed by Fustero and co-workers synthetize, where imidoyl chlorides obtained through CM and subsequent reduction were subjected to Dieckmann condensation, is also capable of furnishing a 7-membered ring system. ${ }^{134}$ As shown in Scheme 14, cyclisation of 65 , in this case, resulted in compound 66 as a tautomeric mixture with a 3:2 ratio. In the final step, chemo- and stereoselective reduction afforded fluorinated cis-2-aminocycloheptane carboxylic acid derivative $( \pm)-67$.

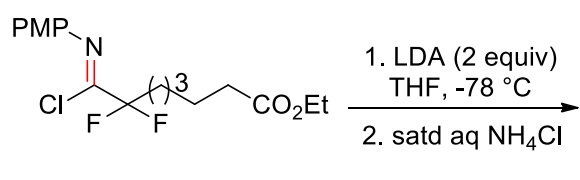

65

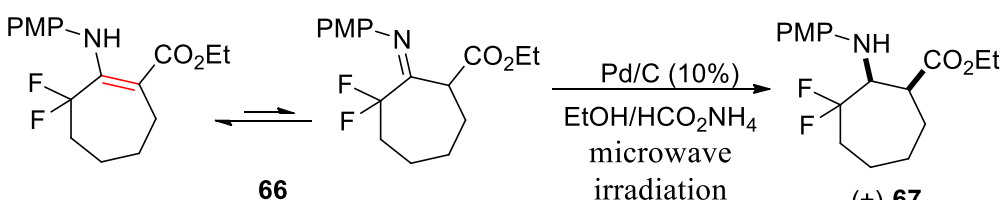

$( \pm)-67$

Scheme 14.

In 2003, the Fustero's group published a study where 7-membered cycles such as compounds ( \pm )-71 and ( \pm )-73 were synthesised exclusively through RCM (Scheme 15). ${ }^{136}$ In this approach fluorinated imidoyl chloride 52a prepared earlier (see Scheme 10) was submitted to condensation with enolate of 4-pentenoic acid ethyl ester 68 to yield $\beta$-imino ester $( \pm)-69$. Next, cyclisation was executed with both first- and second-generation Grubbs catalysts where the latter afforded a better performance. The resulting tautomeric mixture 70, in which the imino 
form dominated, was reduced with $\mathrm{NaCNBH}_{3}$ in a diastereoselective fashion to afford fluorinated cis $\beta$-amino acid derivative $( \pm)$-71 with 7 -membered ring as the sole product. Furthermore, the synthesis of trans derivative $( \pm)-\mathbf{7 3}$ could also be accomplished. Namely, reduction of ( \pm -69 with $\mathrm{NaCNBH}_{3}$ gave a 1:1 mixture of syn and anti diastereomeric open-chain $\beta$-amino esters $( \pm)$-72a and $( \pm)$-72b, and this mixture gave the desired compound through RCM.

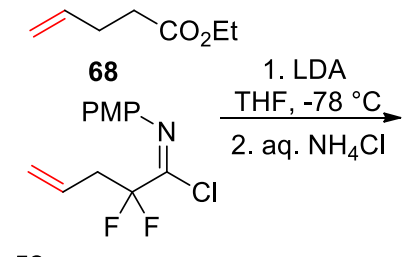

$52 a$

PMP: p-methoxyphenyl

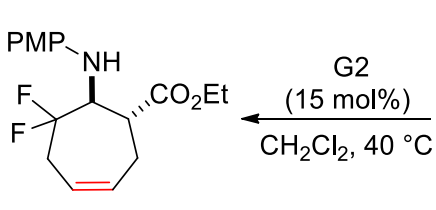

$( \pm)-73$

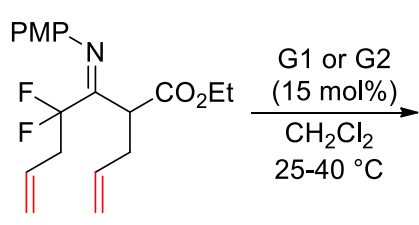

$( \pm)-69$

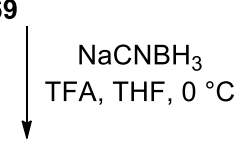

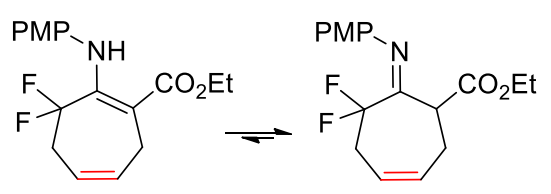

70
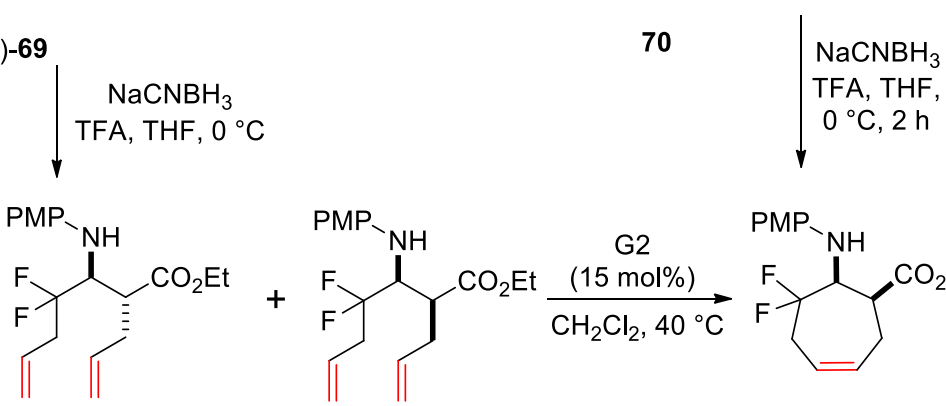

anti $( \pm)-72 b$

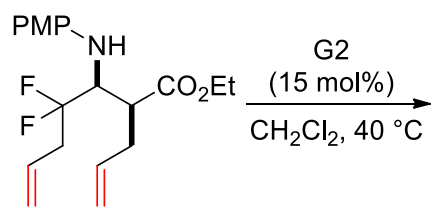

$\operatorname{syn}( \pm)-72 a$
$\mathrm{PMP}_{-\mathrm{NH}}$<smiles>CCOC(=O)C1CC=CCC(F)(F)C1N</smiles>

$( \pm)-71$

Scheme 15.

For the preparation of $\beta$-turn mimetics, Ohkubo and co-workers developed a general procedure towards the exclusive synthesis of larger 7-, 8-, and 9-membered cyclic $\beta^{2}$-amino acid derivatives via RCM (Scheme 16). ${ }^{137}$ The acyclic dienes were prepared in three consecutive steps from readily available aliphatic amines where the alkenyl chain length predetermined ring sizes during cyclisation. $\beta$-Amino ester skeletons were formed by conjugate addition of amines 74a-c to ethyl acrylate 47. Subsequent $N$-protection followed by allylation at the $\alpha$-position resulted in the corresponding precursors $( \pm)$-76a-c. In the case of $( \pm)-76 \mathbf{a}$ and $( \pm)-\mathbf{7 6 b}$, cyclisation smoothly afforded the desired 7- and 8-membered cycloalkene derivatives $( \pm)$-77a and $( \pm)-\mathbf{7 7 b}$, respectively. During the cyclisation of $( \pm)-\mathbf{7 6} \mathbf{c}$, because of olefin bond isomerisation of the starting material, $( \pm)$-777 $\mathbf{c}$ and $( \pm)$-77b were formed together in a 4:1 ratio. Saturated cyclic $\beta^{2}$-amino acid derivatives $( \pm)$-78a-c were synthesised by reduction of the $\mathrm{C}-\mathrm{C}$ double bonds. By slightly modifying the synthetic route discussed above, the authors were also able to prepare $N$-Boc protected 7 - and 8-membered cyclic $\beta^{2}$-amino acids in enantiopure form. 


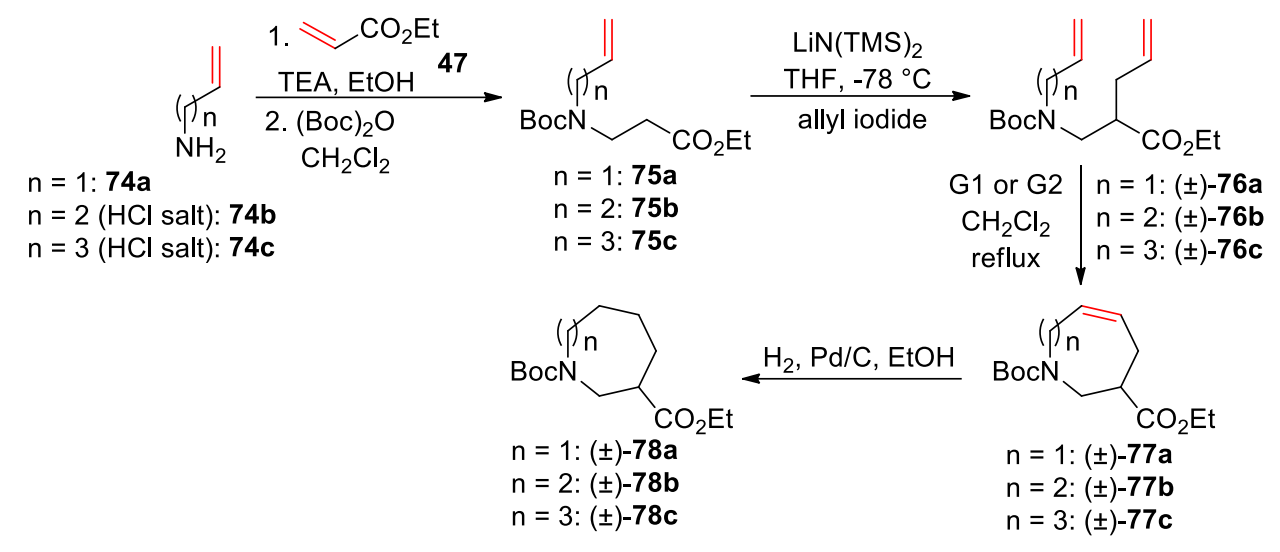

Scheme 16.

\subsection{Synthesis of $\beta$-amino acids with condensed ring systems by ring-rearrangement metathesis (RRM)}

In the following short section, the usefulness of domino and tandem metathesis reactions in the synthesis of $\beta$-amino acid derivatives with condensed ring will be exemplified. These transformations allow the rapid and safe syntheses of highly diverse fused carbo- or heterocyclic ring systems through the rearrangement of the $\mathrm{C}-\mathrm{C}$ double bonds. A frequently applied sequence in these cases is the intramolecular ring-opening/ring-closing protocol. Thanks to their high ring strain, bridged bicyclic frameworks such as norbornene and oxanorbornene provide a great possibility toward condensed 5- or 6-membered carbo- or heterocyclic systems. ${ }^{115-116}$

Winkler et al. utilised the benefits of the oxanorbornene framework for the syntheses of novel tri- and pentaheterocyclic ring systems via tandem metathesis. ${ }^{138}$ First, metathesis substrates were prepared from bicyclic oxanorbornene amino ester $\mathbf{7 9}$ by changing the methyl ester to allyl ester $\mathbf{8 0}$ followed by $N$-allylation to give $\mathbf{8 1}$ or $N$-allylation to $\mathbf{8 2}$ and subsequent creation of an $N$-allylamide moiety (product $\mathbf{8 3}$, Scheme 17). Ring-opening metathesis of the strained oxabicyclo ring of $\mathbf{8 1}$ afforded reactive intermediate $\mathbf{8 4}$ with four terminal alkene functions. It immediately reacted further by RCM to form condensed tricyclic $\beta$-amino acid derivative 85. Analogous tandem metathesis of precursor 83 gave compound 86 a lactam analogue of $\mathbf{8 5}$. 


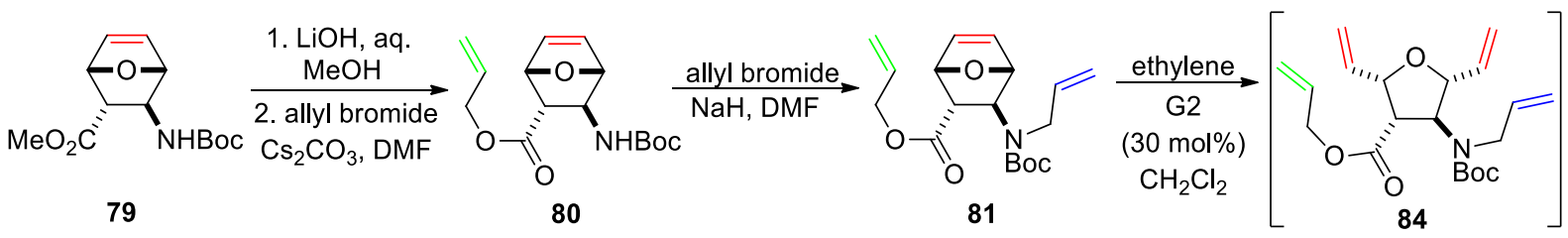

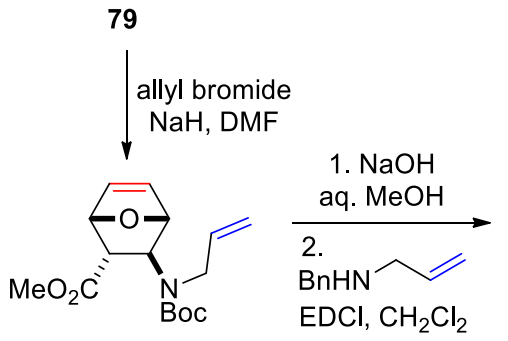

82

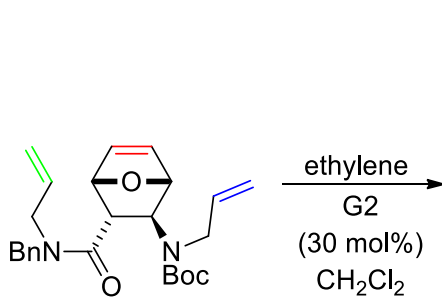

83

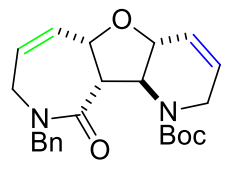

86

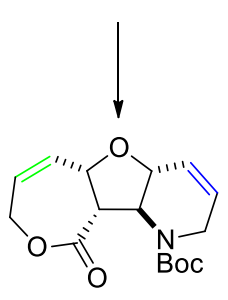

85

Scheme 17.

Pentacyclic product 91 can be synthesised in a similar fashion, although it requires a very high catalyst loading during the ROM/RCM step (Scheme 18). In this case, metathesis precursor 89 was prepared by reductive amination of aldehyde $\mathbf{8 8}$ with $O$-allylated amino ester 87 prepared previously followed by $N$-tosylation. An important role of the tosyl group is to influence the rotational equilibrium of $\mathbf{9 0}$ ensuring the presence of the cis rotamer needed for the RCM step.

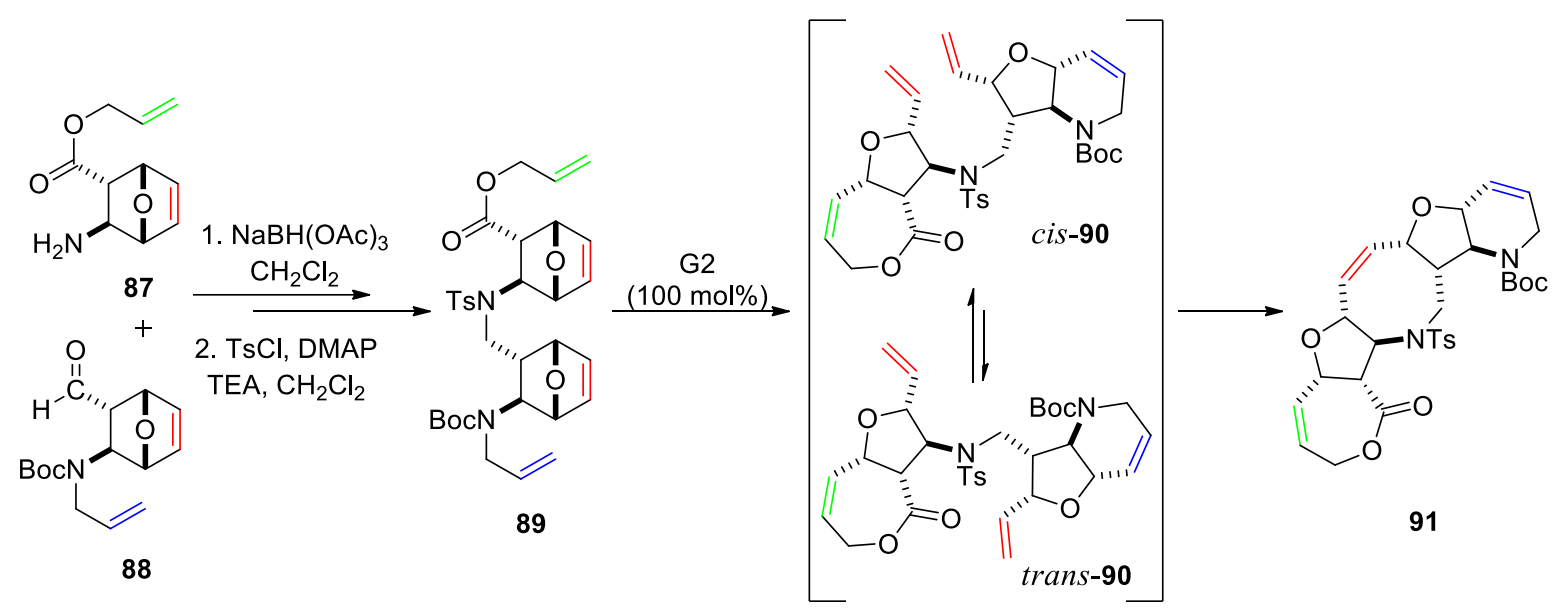

Scheme 18.

Nadany and Mckendrick applied a similar pathway towards constrained bicyclic $\beta$-amino acid derivatives. However, in these cases, only a single ring closure occurred (Scheme 19). ${ }^{139}$ To avoid the chelate formation with participation of the catalyst, carbonyl oxygen and the nitrogen atom, trans $N$-tosylated $\beta$-amino esters were utilised as starting materials in all cases. First, compound ( \pm )-92 was reacted with allyl bromide to produce metathesis substrate $( \pm)-\mathbf{9 3}$. Next, RRM of trans $\beta$-amino ester ( \pm -93 in the presence of ethylene and G1 catalyst readily afforded the desired nitrogen-containing condensed heterocycle ( \pm -94 in tandem fashion. 
Domino metathesis reaction of the previously prepared homologous $( \pm)-\mathbf{9 6},( \pm)-\mathbf{9 8},( \pm)-\mathbf{1 0 0}$, and ( \pm -102 allows the formation, respectively, of 5,6-fused ( \pm )-97, 5,7-fused $( \pm)-\mathbf{9 9}$, and $( \pm)$-103, and 5,8-fused bicyclic $\beta$-amino acid esters $( \pm)$-101.

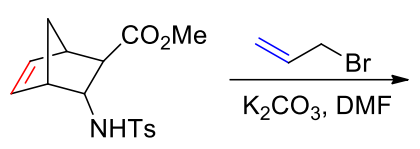

(士)-92

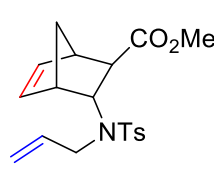

(土) -93

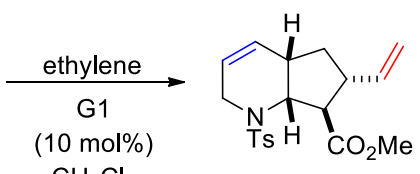

$\mathrm{CH}_{2} \mathrm{Cl}_{2}$

(士)-94

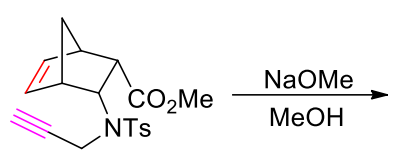

(士)-95

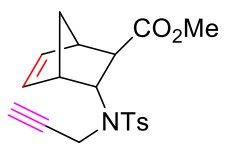

(士)-96

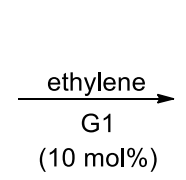

$\mathrm{CH}_{2} \mathrm{Cl}_{2}$<smiles>C=CC1=CC2CC(C=C)C(C(C)OC)C2(N)NC1</smiles>

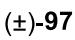

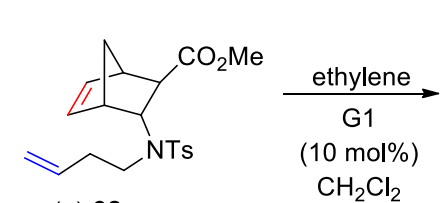

(士)-98

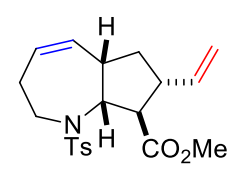

(土)-99

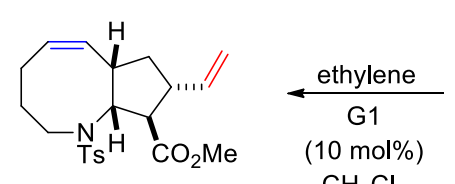

(士)-101

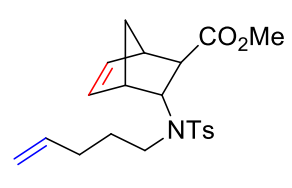

(士)-100

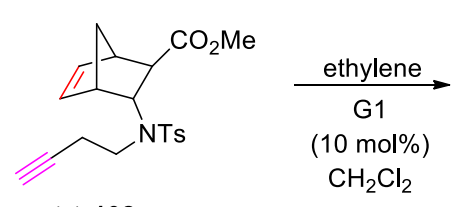

(士)-102

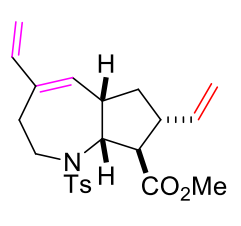

(土)-103

Scheme 19. 


\section{RESULTS AND DISCUSSION}

\subsection{Stereocontrolled one-step synthesis of difunctionalised cispentacin and transpentacin derivatives through ring-opening metathesis (ROM) of norbornene $\beta$-amino acids}

Our synthetic strategy towards difunctionalised cispentacin and transpentacin derivatives was based on the ROM reactions of constrained unsaturated bi- and tricyclic ring systems driven by the release of their high ring strain. For this purpose, norbornene $\beta$-amino esters were excellent candidates. In order to increase the number of achievable cispentacin stereoisomers, some stereoisomeric bicyclic precursors were synthesised. In the first step metathesis substrate compound $( \pm)-\mathbf{1 0 7}$ was prepared by the well-known pathway from readily available starting materials. The synthetic strategy started with the stereoselective addition of chlorosulfonyl

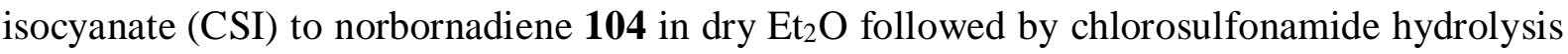
with $\mathrm{Na}_{2} \mathrm{SO}_{3}$ resulting exclusively in exo-lactam $( \pm)-\mathbf{1 0 5}$ as depicted in Scheme 20. ${ }^{140}$

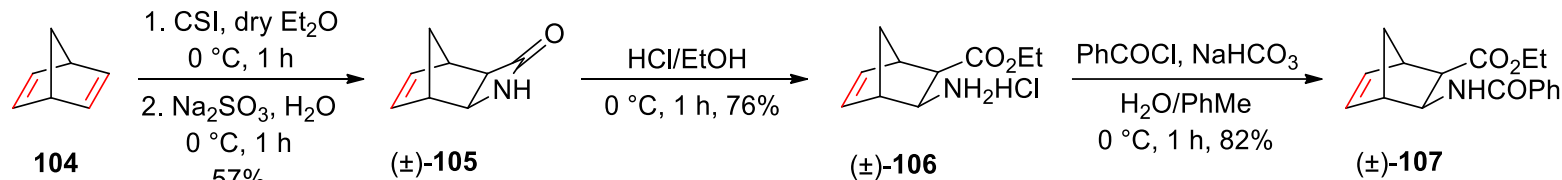

Scheme 20.

In the next step, the 4-membered heterocycle was opened by $\mathrm{HCl} / \mathrm{EtOH}$ and subsequent benzoyl protection of the amino group gave the corresponding unsaturated bicyclic target compound $( \pm)-107 .{ }^{55}$ diendo-Norbornene $\beta$-amino ester $( \pm)$-112 was achievable in a slightly different route as shown in Scheme 21. First, endo-anhydride $( \pm)-\mathbf{1 1 0}$ was prepared via Diels-Alder reaction between cyclopentadiene 108 and maleic anhydride 109. Next, ring-opening reaction of the heterocycle with $\mathrm{NH}_{3}$ followed by Hoffmann degradation smoothly afforded bicyclic $\beta$-amino acid $( \pm)-\mathbf{1 1 1} .{ }^{139}$ Finally, the carboxyl function was esterified with $\mathrm{SOCl}_{2} / \mathrm{EtOH}$ and subsequent benzoylation gave desired metathesis substrate diendo-norbornene $\beta$-amino ester $( \pm)-\mathbf{1 1 2} .{ }^{56}$
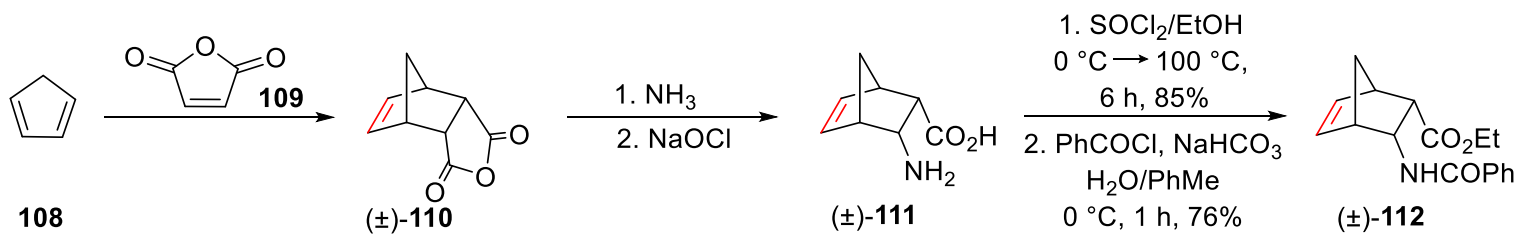

$( \pm)-112$

Scheme 21. 
Next, epimerised derivatives of $( \pm)$-107 and $( \pm)$-112 were prepared by their treatment with $\mathrm{NaOEt}$ in $\mathrm{EtOH}$. The reaction afforded the so-called endo-exo ( \pm )-113 and exo-endo $( \pm)$-114 compounds in which the ester and the amide group are trans to each other. With the four bicyclic metathesis substrates in hand $[( \pm)-\mathbf{1 0 7},( \pm)-\mathbf{1 1 2},( \pm)-\mathbf{1 1 3}$, and $( \pm)-\mathbf{1 1 4}]$, their ring-opening metathesis reaction was studied toward the preparation of functionalised cispentacin derivatives. It was found that racemic 3,5-divinylated amino ester stereoisomers $( \pm)$-115, $( \pm)$-116, $( \pm)-\mathbf{1 1 7}$, and $( \pm)$-118, which differ in the relative configuration of the C-1 and/or C-2 atom, were readily accessible through ROM of the corresponding starting bicyclic compounds in the presence of ethylene and metathesis catalyst (Scheme 22).

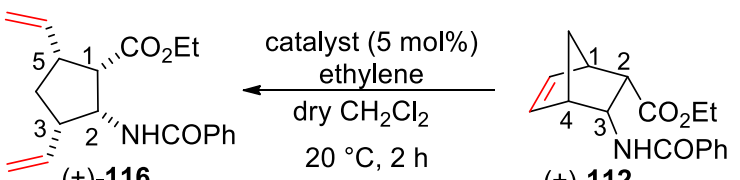

$( \pm)-116$

$( \pm)-112$

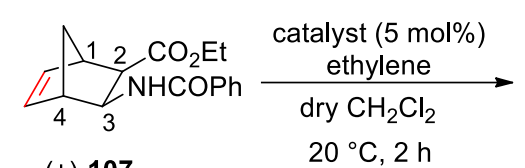

$( \pm)-107$

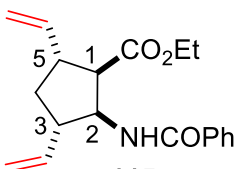

$( \pm)-115$

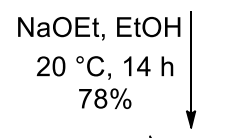

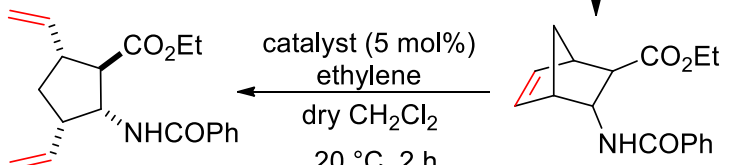

$( \pm)-118$

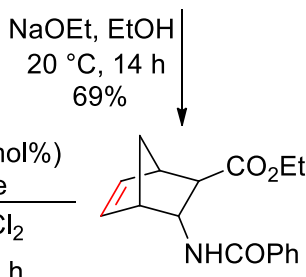

$( \pm)-114$

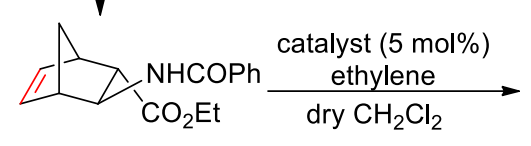

$( \pm)-113$ $20^{\circ} \mathrm{C}, 2 \mathrm{~h}$

Scheme 22.

Metathesis reactions were performed systematically in anhydrous $\mathrm{CH}_{2} \mathrm{Cl}_{2}$ with ethylene under argon atmosphere in the presence of a metathesis catalysts (G1, G2, HG1, or HG2) for 2 h. In the case of diexo- and diendo- $\beta$-amino ester metathesis substrates $( \pm)-\mathbf{1 0 7}$ and $( \pm)-\mathbf{1 1 2}$, clear general correlation between the yield and the used catalyst was not found, although HG2, in both cases, was slightly more effective than the other catalysts. Notably, however, yields in the case of endo-exo $( \pm)-\mathbf{1 1 3}$ and exo-endo $( \pm)-\mathbf{1 1 4}$ derivatives found with first generation catalysts were more than two times higher than those by second generation ones (see Table 1). The best yields (80\% and 68\%) were achieved also with endo-exo $( \pm)-\mathbf{1 1 3}$ and exo-endo $( \pm)-\mathbf{1 1 4}$ compounds. Lower yields found with diexo and diendo derivatives $( \pm)-\mathbf{1 0 7}$ and $( \pm)-\mathbf{1 1 2}$ may be explained by chelation of the catalyst. Specifically, the cis arrangement of the amide and ester groups in these substrates enable their simultaneous coordination to the Ru centre, which greatly reduces the efficiency of the catalyst. It is worth mentioning that the bicyclic starting materials have four asymmetric centres, and since these atoms are unaffected during the transformation, the chiral information is transferred to the final products. Notably, compound $( \pm)-\mathbf{1 1 5}$ was synthesised earlier from the same starting material $( \pm)-\mathbf{1 0 7}$ on another synthetic route by our research group in a $30 \%$ overall yield. ${ }^{55}$ 
The present method not only afforded significantly higher yields and saved a number of steps, but avoided the use of highly toxic chemicals such as $\mathrm{OsO}_{4}$. Moreover, all-cis divinylated amino ester $( \pm)$-116 could not be synthesised by the previously mentioned method because of base-induced epimerisation. ${ }^{56}$

Table 1. Isolated yields for $( \pm)-\mathbf{1 1 5},( \pm)-\mathbf{1 1 6},( \pm)-\mathbf{1 1 7}$, and $( \pm)-\mathbf{1 1 8}$ in ROM reactions with various catalysts.

\begin{tabular}{|c|c|c|c|c|c|c|c|c|c|}
\hline Catalyst & \multirow{2}{*}{ G1 } & \multirow{2}{*}{ G2 } & \multirow{2}{*}{ HG1 } & \multirow{2}{*}{ HG2 } & Catalyst & \multirow{2}{*}{ G1 } & \multirow{2}{*}{ G2 } & \multirow{2}{*}{ HG1 } & \multirow{2}{*}{ HG2 } \\
\hline Products & & & & & Products & & & & \\
\hline & \multirow[t]{2}{*}{$37 \%$} & \multirow[t]{2}{*}{$33 \%$} & \multirow[t]{2}{*}{$38 \%$} & \multirow[t]{2}{*}{$41 \%$} & & \multirow[t]{2}{*}{$6 \%$} & \multirow[t]{2}{*}{$26 \%$} & \multirow[t]{2}{*}{$29 \%$} & \multirow[t]{2}{*}{$31 \%$} \\
\hline$( \pm)-\mathbf{1 1 5}$ & & & & & $( \pm)-116$ & & & & \\
\hline & \multirow[t]{2}{*}{$46 \%$} & \multirow[t]{2}{*}{$20 \%$} & \multirow[t]{2}{*}{$80 \%$} & \multirow[t]{2}{*}{$28 \%$} & & \multirow[t]{2}{*}{$68 \%$} & \multirow[t]{2}{*}{$29 \%$} & \multirow[t]{2}{*}{$45 \%$} & \multirow[t]{2}{*}{$16 \%$} \\
\hline$( \pm)-\mathbf{1 1 7}$ & & & & & $( \pm)-\mathbf{1 1 8}$ & & & & \\
\hline
\end{tabular}

A possible pathway for 3,5-divinylated final products is depicted in Figure 6. Because of the high concentration of ethylene, the first step of the mechanism is the formation of reactive methylidene complex Ru-I from the so-called pre-catalyst (HG2) by ligand exchange (metathesis reaction). In the next step, $[2+2]$ cycloaddition occurs between norbornene $\beta$-amino acid derivatives and newly formed carbene Ru-I affording the corresponding metallacyclobutane Ru-II condensed with the bicyclic skeleton. Opening of the strained tricyclic framework takes place in the cycloreversion step by redistribution of the electrons forming new alkylidene complex Ru-III. Finally, another cycloaddition reaction of newly formed cyclic 5-membered Ru-containing $\beta$-amino ester Ru-III with ethylene takes place followed by cycloreversion resulting in the desired ring-opened product. The original $\mathrm{Ru}$-methylidene complex Ru-I is reformed in this step and it can start the catalytic cycle again. 
Formation of Ru-containing methylidene complex

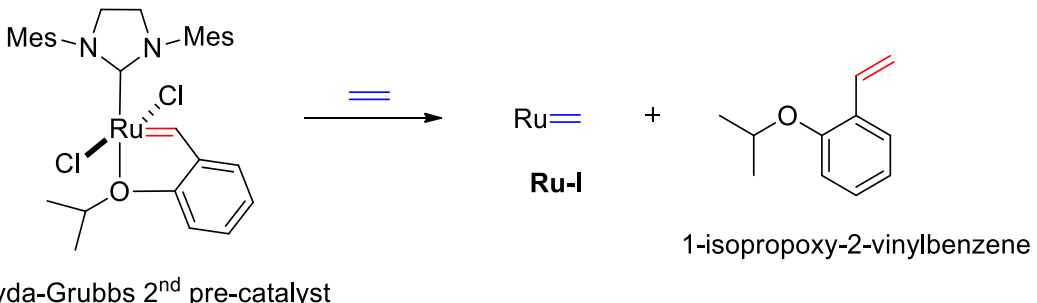

Hoveyda-Grubbs $2^{\text {nd }}$ pre-catalyst

Catalytic cycle of norbornene $\beta$-amino acid derivative

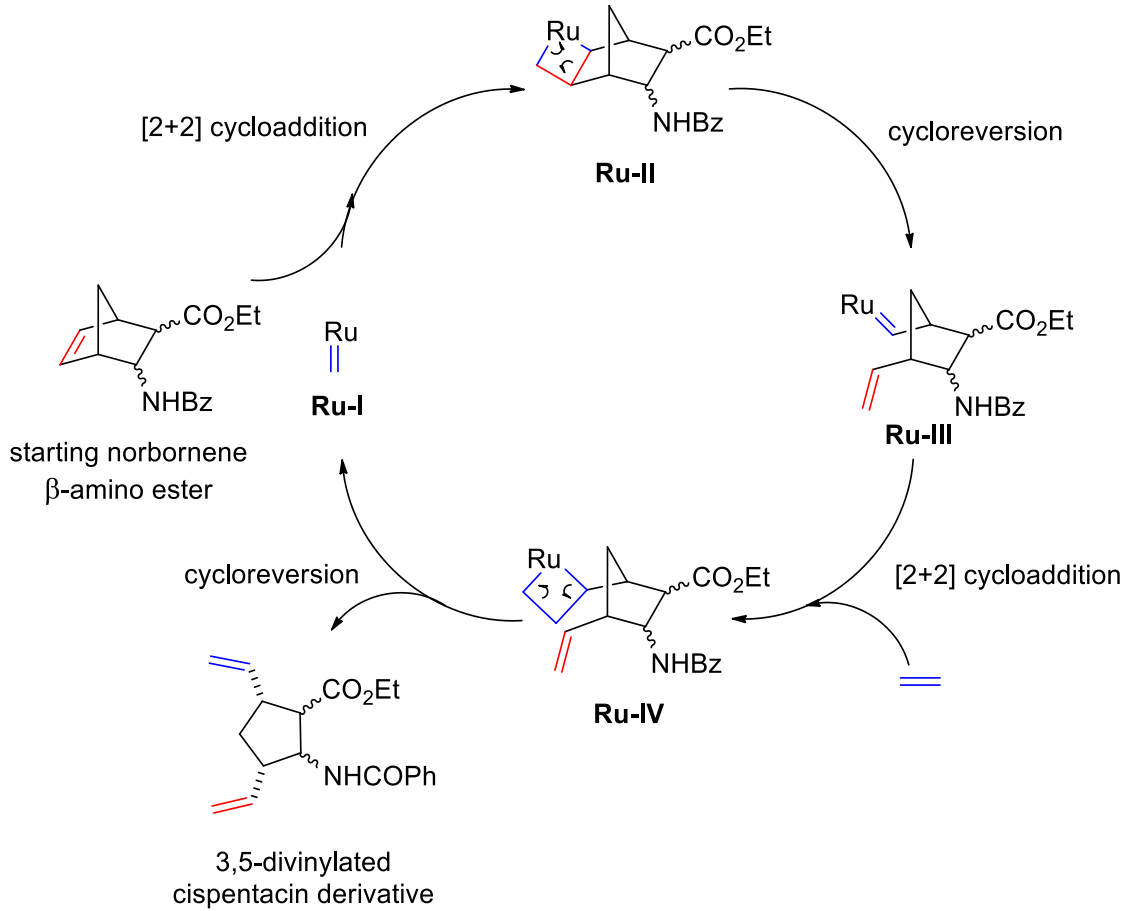

Figure 6.

The above detailed synthetic approach was extended to the preparation of optically pure target substances (+)-115 and (-)-117 (Scheme 23). First, optically active $\beta$-lactam (+)-105 was prepared by lipolase-catalysed enantioselective enzymatic ring-opening of racemic azetidinone $( \pm)-105 .{ }^{141}$ Next, metathesis precursor $(+)-107$ was synthesised in an enantiomer-enriched form via ethanolysis followed by benzoylation. Similar to the racemates, compound (+)-107 was submitted to epimerisation by $\mathrm{NaOEt}$ in $\mathrm{EtOH}$ to afford endo-exo- $\beta$-amino ester derivative (-)-113 in good yield. Finally, ROM reactions with HG1 catalyst with ethylene under argon atmosphere were executed to obtain divinylated cispentacin and transpentacin compounds (+)-115 and (-)-117. The e.r. of optically active esters (+)-115 and (-)-117 were determined by chiral HPLC analysis and found to be 95:5 in both cases. 


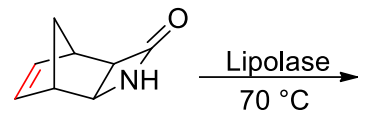

$( \pm)-105$

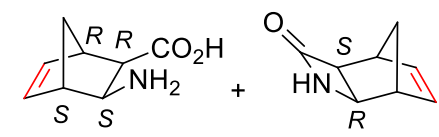

$(-)-119$

$(+)-105$

$$
\begin{array}{c|c}
\begin{array}{c}
\text { 1. } \mathrm{HCl} / \mathrm{EtOH} \\
0{ }^{\circ} \mathrm{C}, 1 \mathrm{~h}
\end{array} & \begin{array}{c}
\text { 2. } \mathrm{PhCOCl}, \mathrm{NaHCO}_{3} \\
\mathrm{PhMe}, 0{ }^{\circ} \mathrm{C} \\
\text { for two steps: } 56 \%
\end{array}
\end{array}
$$

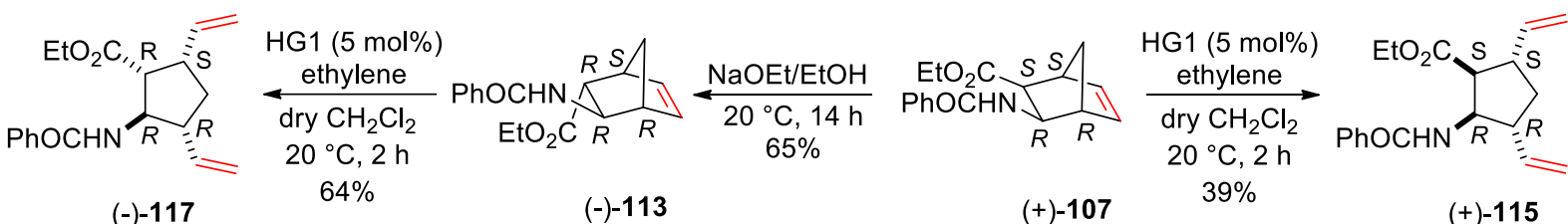

Scheme 23.

In view of the relevance of oxygen-containing cyclic $\beta$-amino acids, the stereocontrolled one-step method applied earlier was extended toward divinylated tetrahydrofuran $\beta$-amino esters as depicted in Scheme $24 .^{2}$ Thus, oxanorbornene amino ester $( \pm)$-122 was prepared in two steps from the corresponding diexo- $\beta$-amino acid hydrochloride salt $( \pm)$-120 similar to compound $( \pm)$-112. Epimerisation of $( \pm)-\mathbf{1 2 2}$ with $\mathrm{NaOEt}$ in $\mathrm{EtOH}$ at $20{ }^{\circ} \mathrm{C}$ gave endo-exo derivative $( \pm)-\mathbf{1 2 3}$ in moderate yield. The synthesis of novel divinylated tetrahydrofuran $\beta$-amino esters $( \pm)$-124 and ( \pm -125 was achieved in good yields from the corresponding bicyclic starting materials $( \pm)-\mathbf{1 2 2}$ and $( \pm)-\mathbf{1 2 3}$ in ROM reaction with the conservation of the four stereogenic centres. The metathesis reactions were performed in dry $\mathrm{CH}_{2} \mathrm{Cl}_{2}$ under ethylene and argon atmosphere in the presence of HG1 catalyst (Scheme 24).

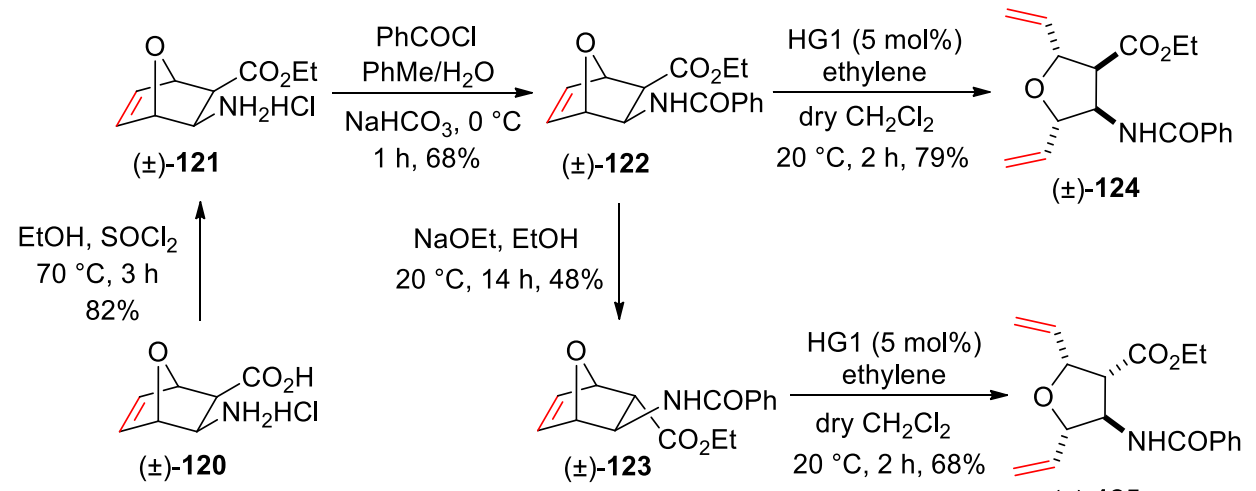

$( \pm)-125$

Scheme 24. 


\subsection{Stereocontrolled one-step synthesis of difunctionalised azetidinones and $\beta$-amino acid derivatives from condensed ring $\beta$-lactams by ring-opening metathesis (ROM)}

Novel functionalised azetidin-2-ones and $\beta$-amino acid derivatives were synthesised according to our earlier strategy. The driving force of these reactions is the formation of thermodynamically more stable molecules, which have lower ring strain than the bi- or tricyclic starting materials. ${ }^{15-116}$ During these ring-opening processes mediated by $\mathrm{Ru}$-alkylidene complexes, epimerisation was not observed, that is the stereochemistry of the starting $\beta$-lactams was conserved and determined the configuration of the chiral centres in the end-products. In the first step, tricyclic $\beta$-lactam ( \pm )-105 was synthesised by the well-known method, via the addition of CSI to norbornadiene $\mathbf{1 0 4}$ in dry $\mathrm{Et}_{2} \mathrm{O}$ at $0{ }^{\circ} \mathrm{C}$, followed by chlorosulfonamide hydrolysis with $\mathrm{Na}_{2} \mathrm{SO}_{3}$ (Scheme 25). ${ }^{2}$

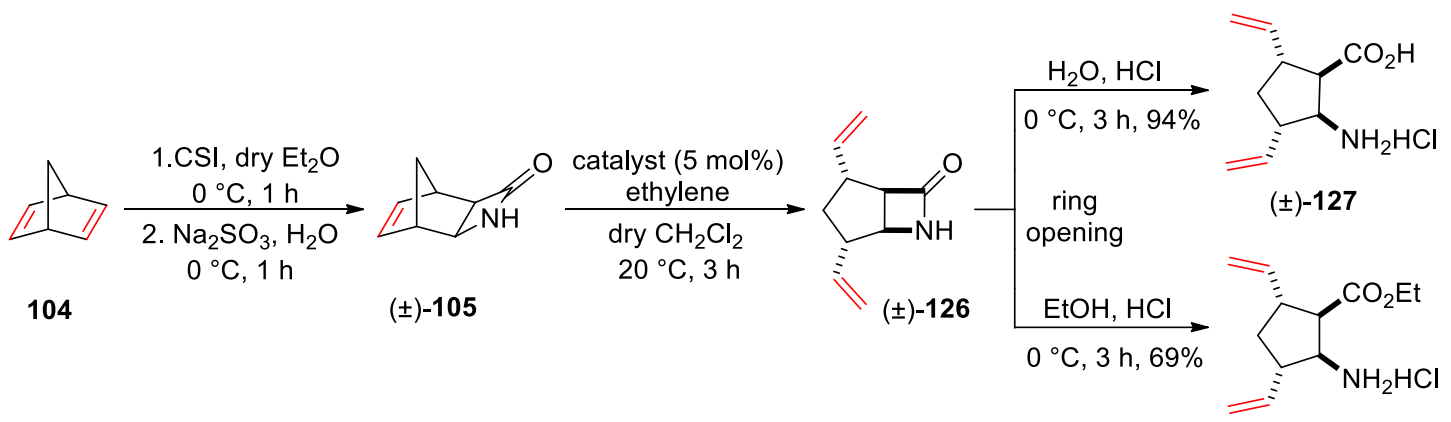

$( \pm)-128$

Scheme 25. Syntheses of divinylated cispentacin derivatives ( \pm -127 and ( \pm -128. Yields of $( \pm)-\mathbf{1 2 6}$ G1: 80\%; G2: $5 \%$; $\mathrm{HG} 1:$ 70\%; and $\mathrm{HG} 2: 10 \%$.

Resulting diexo- $\beta$-lactam $( \pm)-\mathbf{1 0 5}$ was subjected to ROM reaction in the presence of various commercially available first and second generation Ru-based catalysts (G1, G2, HG1, and HG2) with ethylene under argon atmosphere with stirring in dry $\mathrm{CH}_{2} \mathrm{Cl}_{2}$ at $20{ }^{\circ} \mathrm{C}$. Divinyl-substituted $\beta$-lactam $( \pm)$-126 was formed in high yields with the first generation catalysts, while second generation catalysts preferred the formation of polymeric products. In the final step, racemic cispentacin derivatives $( \pm)$-127 and $( \pm)$-128 containing valuable olefinic bonds were accessed through opening of the 4-membered heterocycle. Thus, norbornadiene-derived $\beta$-lactam $( \pm)$-126 was subjected to hydrolysis or ethanolysis in a stereocontrolled fashion to afford the corresponding cyclic 5 -membered $\beta^{2,3}$-amino acid $( \pm)$-127 or $\beta^{2,3}$-amino ester $( \pm)$-128 as hydrochloride salts in good to excellent yields (Scheme 25). 
The above ring-opening metathesis protocol was extended to the stereocontrolled syntheses of monocyclic $\beta$-lactams with terminal olefin moiety. The synthesis was started with the preparation of the corresponding bicyclic azetidinone framework $( \pm)-\mathbf{1 3 0}$ in two steps from readily available starting materials. 1,5-Cyclooctadiene 129 was reacted with CSI in dry $\mathrm{CH}_{2} \mathrm{Cl}_{2}$ at $0{ }^{\circ} \mathrm{C}$, then hydrolysis with $\mathrm{Na}_{2} \mathrm{SO}_{3}$ gave 1,5-cyclooctadiene-derived $\beta$-lactam $( \pm)$-130 (Scheme 26).

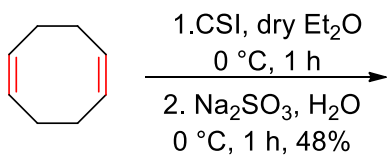

129

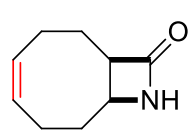

$( \pm)-130$

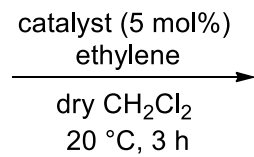

$20{ }^{\circ} \mathrm{C}, 3 \mathrm{~h}$

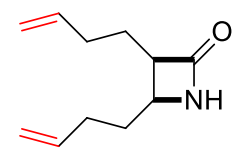

$( \pm)-131$

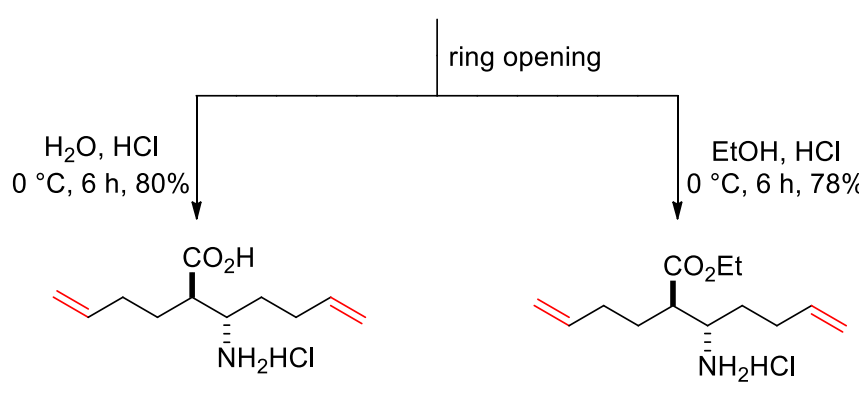

$( \pm)-132$

$( \pm)-133$

Scheme 26.

Racemic monocyclic azetidinone $( \pm)$-131 with alkenyl moieties at the end of the alkyl chains was accessed through metathesis in dry $\mathrm{CH}_{2} \mathrm{Cl}_{2}$ at $20{ }^{\circ} \mathrm{C}$. We have tested again first and second generation $\mathrm{Ru}$-based catalysts (see Table 2 ) with results strikingly similar to those found with norbornene $\beta$-lactam $( \pm)-\mathbf{1 0 5}$. G1 and HG1 catalysts performed well in the ring-opening reaction, while $\mathrm{G} 2$ and $\mathrm{HG} 2$ was too reactive inducing mainly polymerisation resulting in low yields of $( \pm)$-131. Finally, treatment of compound $( \pm)-\mathbf{1 3 1}$ with $\mathrm{HCl} / \mathrm{H}_{2} \mathrm{O}$ at $0{ }^{\circ} \mathrm{C}$ gave the corresponding open-chain $\beta$-amino acid $( \pm)$-132 with the carboxyl and amine functions in anti arrangement. Ethanolysis, in turn, gave open chain $\beta$-amino ester $( \pm)$-133 with the same stereochemistry in a yield of $78 \%$.

Table 2. Isolated yields for $( \pm)-\mathbf{1 3 1}$ and $( \pm)-\mathbf{1 3 6}$ in ROM reactions with various catalysts.

\begin{tabular}{|c|c|c|c|c|c|c|c|c|c|}
\hline Catalyst & \multirow{2}{*}{ G1 } & \multirow{2}{*}{ G2 } & \multirow{2}{*}{ HG1 } & \multirow{2}{*}{ HG2 } & Catalyst & \multirow{2}{*}{ G1 } & \multirow{2}{*}{ G2 } & \multirow{2}{*}{ HG1 } & \multirow{2}{*}{ HG2 } \\
\hline Product & & & & & Product & & & & \\
\hline & \multirow[t]{2}{*}{$75 \%$} & \multirow[t]{2}{*}{$7 \%$} & \multirow[t]{2}{*}{$74 \%$} & \multirow[t]{2}{*}{$17 \%$} & & \multirow[t]{2}{*}{$60 \%$} & \multirow[t]{2}{*}{$25 \%$} & \multirow[t]{2}{*}{$67 \%$} & \multirow[t]{2}{*}{$26 \%$} \\
\hline$( \pm)-131$ & & & & & $( \pm)-136$ & & & & \\
\hline
\end{tabular}


Following our strategy, after the successful ROM in the case of compound $( \pm)-\mathbf{1 3 0}$, it seemed a logical step to prepare 1,3-cyclooctadiene-derived bicyclic $\beta$-lactam $( \pm)-\mathbf{1 3 5}$ a regioisomer of compound $( \pm$ )-130 (Scheme 27). For this purpose, 1,3-cyclooctadiene 134 was reacted with CSI using the well-known pathway to afford the desired metathesis substrate $( \pm)$-135 with high ring strain. During the ring-opening protocol, first generation catalysts proved to be beneficial again, although ring-opening product $( \pm)$-136 was also isolated in modest but acceptable yields in the presence of $\mathrm{G} 2$ and $\mathrm{HG} 2$ in this case (see Table 2).

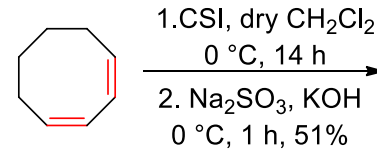

134

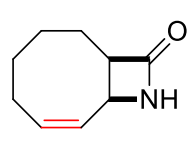

$( \pm)-135$

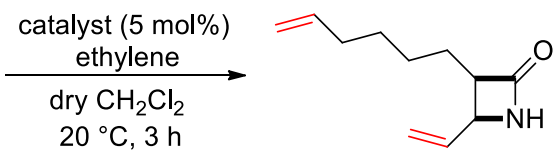

$( \pm)-136$

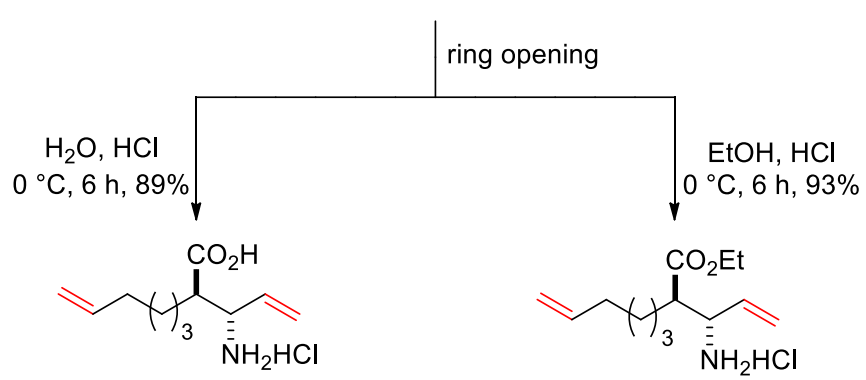

$( \pm)-137$

$( \pm)-138$

Scheme 27.

In the final step, open chain $\beta$-amino acid $( \pm)$-137 and $\beta$-amino ester $( \pm)$-138 bearing valuable olefinic bonds were prepared. 4-Membered heterocycle $( \pm)-\mathbf{1 3 6}$ was opened by $\mathrm{H}_{2} \mathrm{O}$ or EtOH under protic conditions and gave the expected products in excellent yields in both cases. Similar to previous experiences, when compound $( \pm)-\mathbf{1 3 6}$ underwent hydrolysis or ethanolysis, configuration of the two asymmetric centres left unaffected and afforded anti arrangement for the carboxyl and amino hydrochloride groups, as depicted in Scheme 27.

However, besides successful ring-opening metathesis reactions, we have encountered difficulties in some cases (Scheme 28). Attempted ring-opening transformations of cyclopentadiene- or cyclohexadiene-derived $\beta$-lactams $( \pm)-\mathbf{1 3 9},( \pm)-\mathbf{1 4 1}$, and $( \pm)$-143, which have less ring strain, did not furnish the corresponding monocyclic products $( \pm)-\mathbf{1 4 0},( \pm)-\mathbf{1 4 2}$, and $( \pm)$-144. Unfortunately, all metathesis reactions performed in a variety of solvents $\left(\mathrm{CH}_{2} \mathrm{Cl}_{2}\right.$, toluene) at room temperature or under reflux with all catalysts (G1, G2, HG1, and HG2) failed and only the starting materials were detected by TLC monitoring. This may be accounted for by the shift of the ring-opening/ring-closing equilibrium towards recyclisation under metathesis conditions. 
Obviously ring-closing reactions of $( \pm)-\mathbf{1 2 6},( \pm)-\mathbf{1 3 1}$, and $( \pm)-\mathbf{1 3 6}$ are less favoured because of evolving ring strain. Furthermore, the distance between the newly formed terminal alkene moieties further reduces the possibility for the ring closing reaction in the case of cyclooctadiene-derived compounds $( \pm)-\mathbf{1 3 1}$ and $( \pm)-\mathbf{1 3 6} .{ }^{124}$

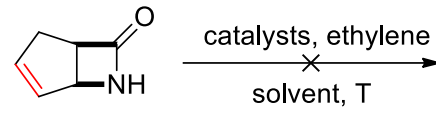

$( \pm)-139$

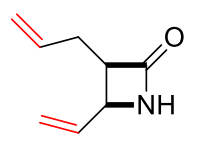

$( \pm)-140$

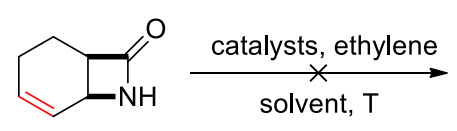

$( \pm)-141$

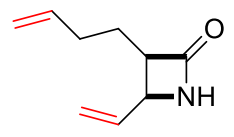

$( \pm)-142$

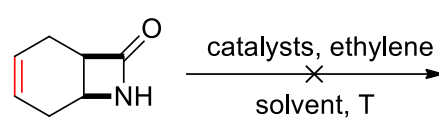

$( \pm)-143$

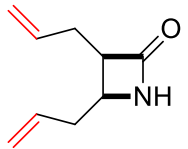

$( \pm)-144$

Scheme 28.

\subsection{Carbon-carbon double bond functionalisation of $\beta$-amino acid derivatives and $\beta$-lactams with $\alpha, \beta$-unsaturated carbonyl compounds through cross metathesis (CM)}

Cross metathesis is a widely applied method for the functionalisation of $\mathrm{C}-\mathrm{C}$ double bonds even in more complex frameworks. ${ }^{142}$ The reaction can proceed in a chemoselective manner and it is usually $E$-selective. ${ }^{120}$ Because of the advantages of metathesis transformations, we planned the functionalisation of terminal alkene moieties of the corresponding $\beta$-lactams or $\beta$-amino acid derivatives synthesised previously through cross metathesis reactions. For this purpose, $\alpha, \beta$-unsaturated carbonyl compounds were used as electron-deficient olefins in the presence of HG2 catalyst. According to the literature, this is an effective cross metathesis protocol. ${ }^{142}$ The two terminal $\mathrm{C}-\mathrm{C}$ double bonds on the ring-opened products were coupled with methyl acrylate 145 and methyl vinyl ketone 148.

First, divinylated transpentacin $( \pm$ )-117 (derived from diexo-norbornane $\beta$-amino ester $( \pm)$-107 by epimerisation followed by ROM) was reacted with methyl acrylate $\mathbf{1 4 5}$ in the presence of $\mathrm{HG} 2$ catalyst in dry $\mathrm{CH}_{2} \mathrm{Cl}_{2}$ at reflux temperature. After the chromatographic separation from the undesired polymeric material, dicoupled product $( \pm)-\mathbf{1 4 6}$ was isolated in $51 \%$ yield (Scheme 29). Importantly, cross metathesis reactions were performed with both first generation Grubbs catalysts, but the desired target compound formed only in trace amounts (monitored by TLC). 
After the successful cross-coupling reaction of compound $( \pm)-\mathbf{1 1 7}$, all-cis divinylated $\beta$-amino ester $( \pm)$-116 was subjected to cross metathesis with methyl acrylate $\mathbf{1 4 5}$ in the presence of HG2 catalyst. Cispentacin derivative $( \pm)-\mathbf{1 4 7}$ with three ester functions was accessed analogously to $( \pm)$-146 in good yield, without epimerisation of the stereogenic centres. It is important to note that compound $( \pm)$-147 was identical to the material prepared earlier on a different synthetic pathway. It may be concluded that the $\mathrm{CM}$ reaction proceeded under stereocontroll with $E$ selectivity, that is the configuration of the newly created $\mathrm{C}-\mathrm{C}$ double bonds in ( \pm -146 and ( \pm -147 also have $E$ geometry. ${ }^{56}$

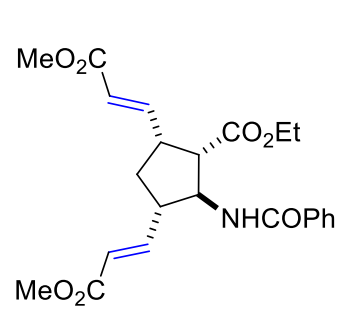

$( \pm)-146$

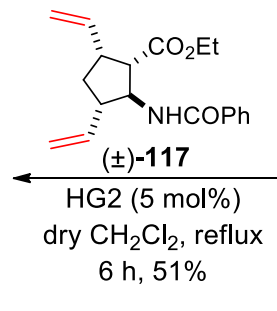

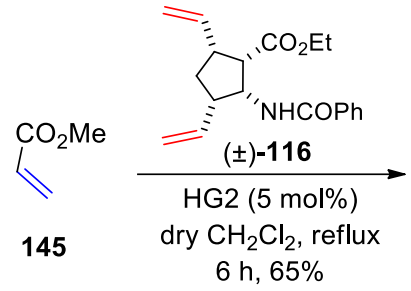

$6 \mathrm{~h}, 65 \%$

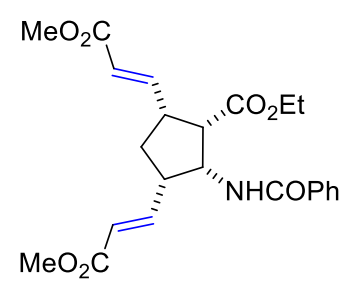

$( \pm)-147$

Scheme 29.

Since cross metathesis reactions worked well in the case of cispentacin derivatives $( \pm)$-116 and $( \pm)$-117, we expanded the scope of the coupling reaction to $\beta$-lactams. Besides of methyl acrylate 145, the terminal alkene moieties were transformed with methyl vinyl ketone 148 as well in these cases. First, bicyclic $\beta$-lactam $( \pm)$-126 (derived from exo-lactam $( \pm)$-105 by $\mathrm{ROM}$ ) was subjected to coupling reactions with $\alpha, \beta$-unsaturated carbonyl compounds 145 and 148 in the presence of HG2 catalyst (Scheme 30). In both cases, after chromatographic purification, cross metathesis products $( \pm)-\mathbf{1 4 9}$ and $( \pm)$-150 with $E$ geometry (high $J$ values, in accordance with earlier literature data) were isolated in moderate to good yields. Cross metathesis reactions were performed in anhydrous solvents such as $\mathrm{CH}_{2} \mathrm{Cl}_{2}$ or toluene with the former giving higher yields in both cases (see Table 3).

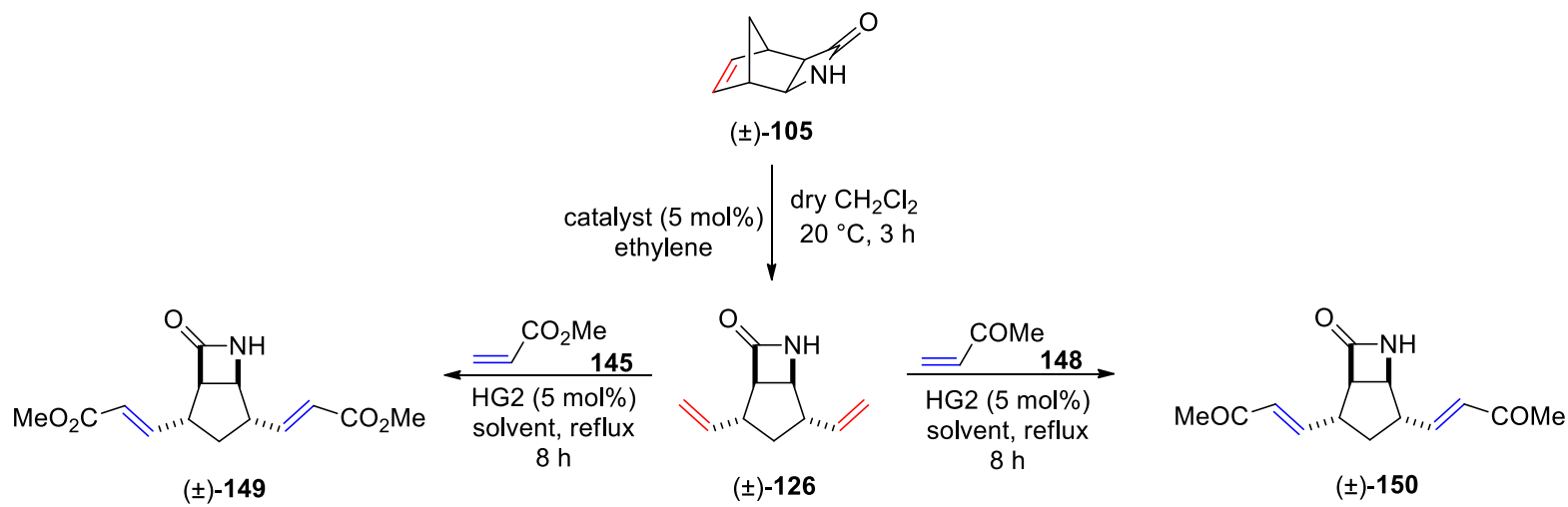

Scheme 30. 
Table 3. Isolated yields for $( \pm)-\mathbf{1 4 9}$ and $( \pm)-\mathbf{1 5 0}$ in CM reactions with HG2 catalyst in different solvents.

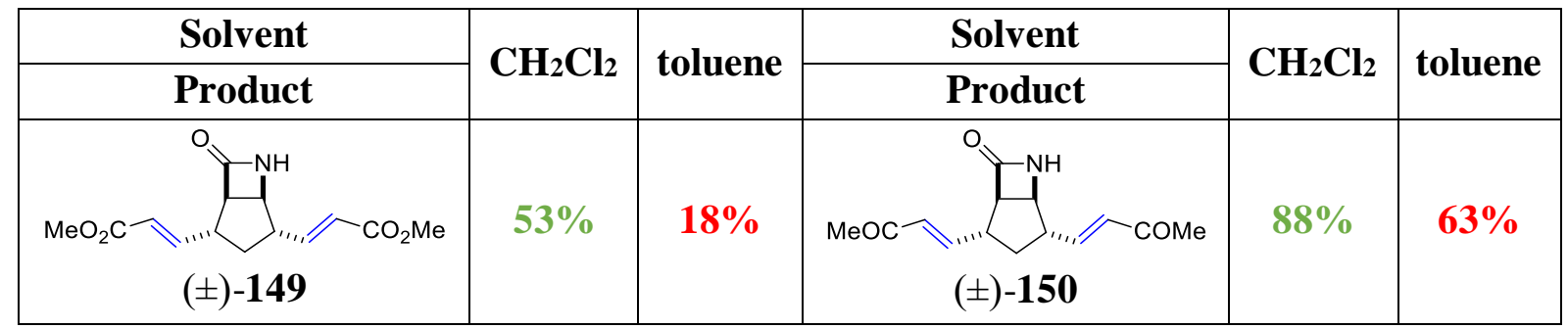

The well-established cross metathesis protocol for the synthesis of highly-functionalised bicyclic $\beta$-lactams was expanded to monocyclic unsaturated $\beta$-lactam derivatives. Ring-opening product $( \pm$-131 prepared previously was coupled with methyl acrylate $\mathbf{1 4 5}$ and methyl vinyl ketone 148 analogously to bicyclic $\beta$-lactam $( \pm)-\mathbf{1 2 6}$ in the presence of HG2 catalyst as depicted in Scheme 31. Coupling reactions smoothly afforded the expected final products $( \pm)-\mathbf{1 5 1}$ and $( \pm)$-152 with $E$ geometry in good yields (see Table 4 ) similar to the formation of products $( \pm)-\mathbf{1 4 9}$ and $( \pm)-\mathbf{1 5 0}$ synthesised previously.

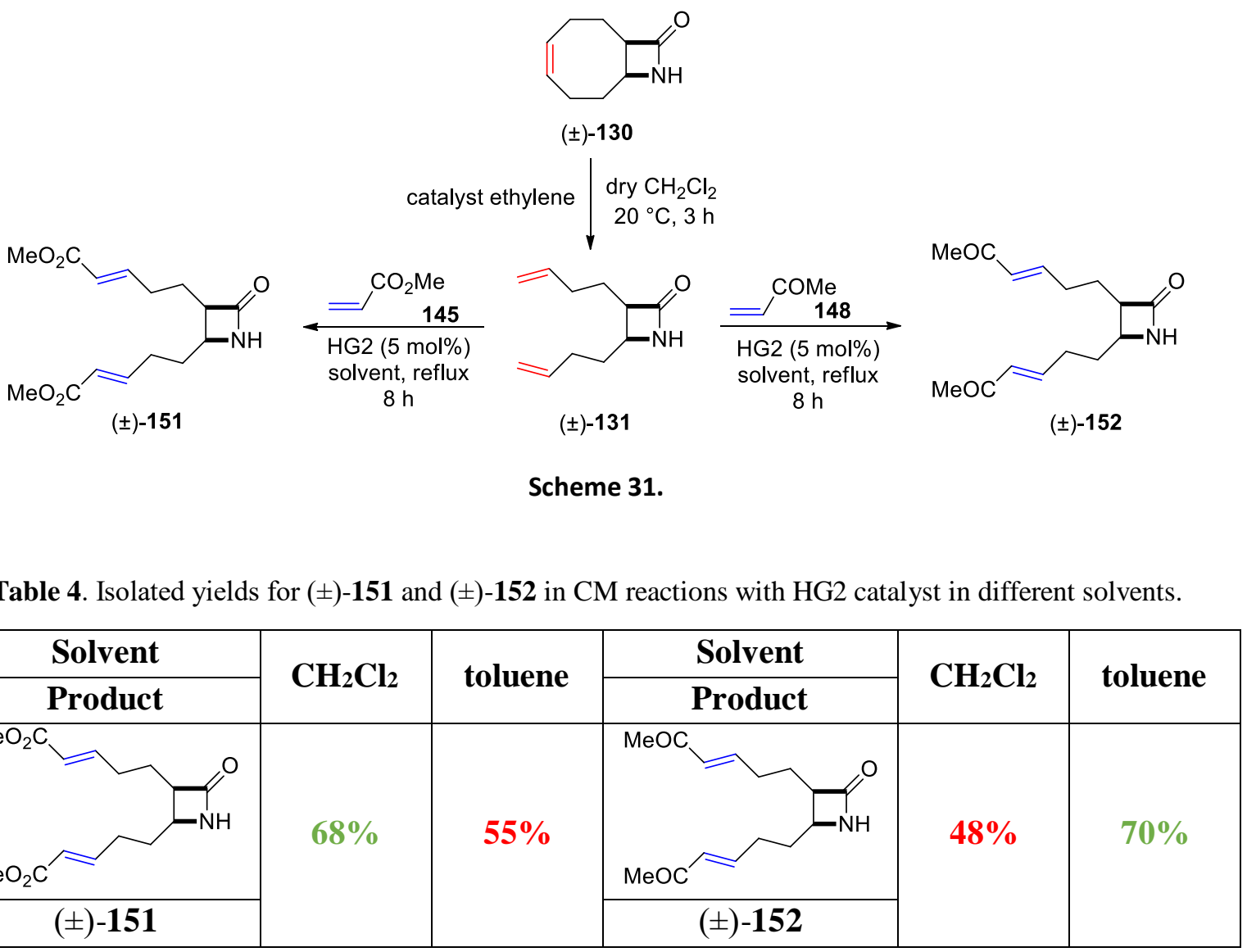


Finally, $\beta$-lactam derivative $( \pm)$-136 [a regioisomer of $( \pm)-\mathbf{1 3 1}$ ] underwent cross metathesis reactions with the previously used electron-deficient olefins 145 and 148. Coupling reactions were carried out in dry toluene or $\mathrm{CH}_{2} \mathrm{Cl}_{2}$ at reflux temperature in the presence of HG2 catalyst (Scheme 32). Di-ester $\beta$-lactam $( \pm)-\mathbf{1 5 3}$ was isolated in $\mathbf{7 3 \%}$ yield when the reaction was performed in toluene, while in the case of its ketone counterpart $( \pm)-\mathbf{1 5 4}$ the use of $\mathrm{CH}_{2} \mathrm{Cl}_{2}$ as solvent resulted in higher yield (see Table 5).

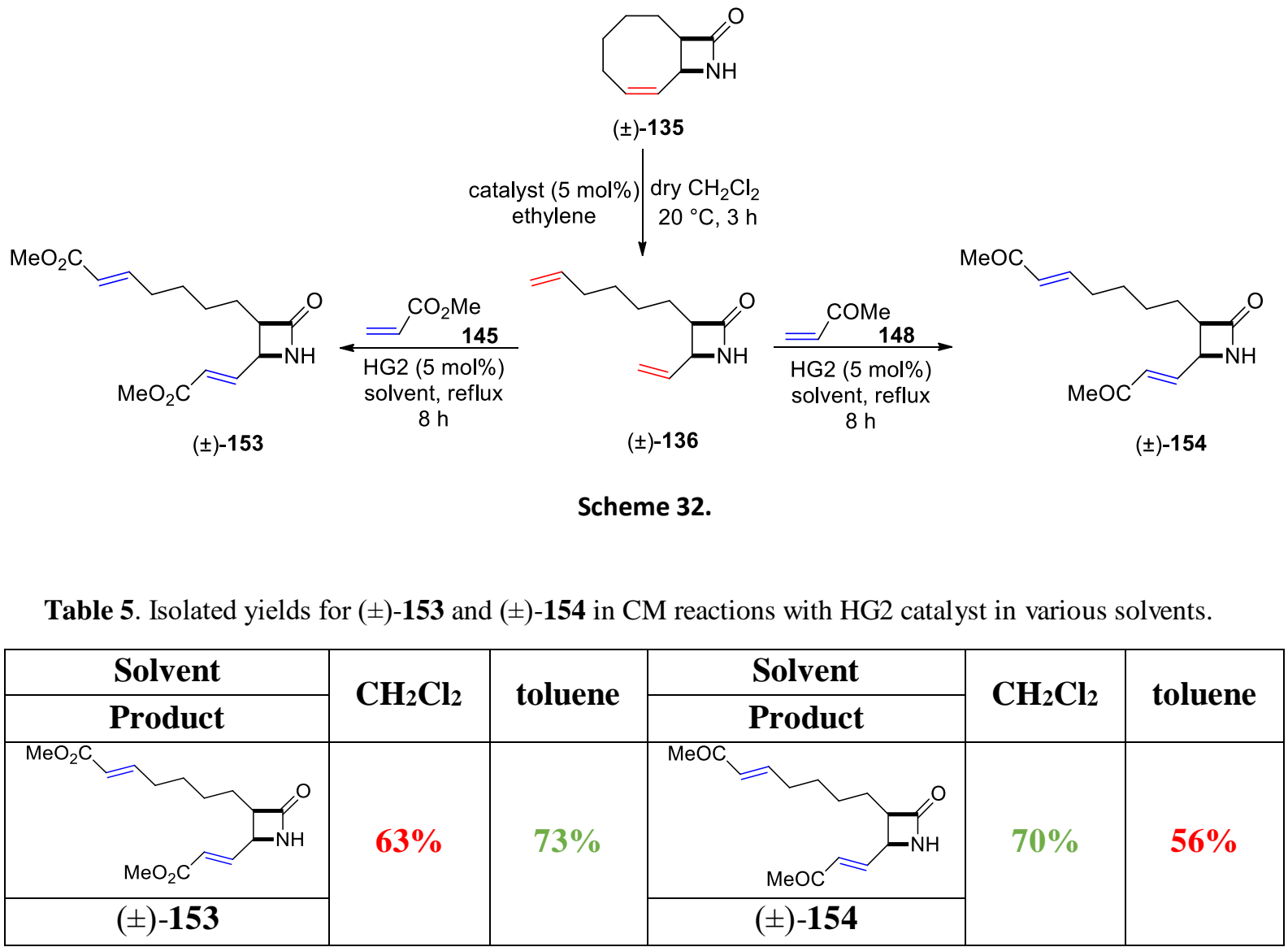

However, it is important to note, that product selectivity during CM reactions is not always predictable, because it is often complicated to determine, which olefin reacts selectively. To solve this problem, Chatterjee et al. compiled a table containing different types of olefins with the appropriate catalysts. ${ }^{142}$ With this new approach in hand, high selectivity is achievable by using the appropriate reagents during the coupling reactions. In their studies the olefins were categorised in four $(+1)$ types based i) on the susceptibility of the starting materials to form homodimers via CM and ii) on the ability of the coupled product toward secondary metathesis reactions. The first group includes olefins, which are able to undergo rapid homodimerisation and the product homodimers possess the same reactivity towards subsequent metathesis reactions as their parent olefins. 
Type II group consists of olefins, which homodimerise slowly and their homodimers are sparingly consumed in secondary coupling reactions. Type III olefins lack the ability to form homodimers but are still able to perform CM reactions with Type I or Type II olefins. Type IV alkenes cannot react in $\mathrm{CM}$ reactions and they have no effect on the catalyst activity. Finally, the +1 category consists of alkenes, which inhibit catalyst activity.

The study determines that selective $\mathrm{CM}$ reaction can be achieved by selecting the appropriate olefins from different types without using large stoichiometric excess of one compound. In our case, the reagents ( $\alpha, \beta$-unsaturated carbonyl compounds) are categorised as Type II olefins, while the substrates in the case of cispentacin and transpentacin derivatives $[( \pm)-\mathbf{1 1 5},( \pm)-\mathbf{1 1 6},( \pm)-\mathbf{1 1 7}$, and $( \pm)-\mathbf{1 1 8}]$ belong to Type III based on our experimental results (homodimer product was not formed but reacted selectively with Type II olefins). Monocyclic unsaturated $\beta$-lactam $( \pm)$-131 (terminal olefin) is categorised as Type I alkene. In contrast, the classification is not straightforward for $( \pm)-\mathbf{1 3 6}$ because of the different alkenyl moieties: the molecule contains both a terminal alkene part and a tertiary allylic position. Despite complications arising from the classification, CM products were isolated in all cases with moderate to good yields (Table 3, 4, 5 and Scheme 29). However, it is worthwhile to mention that, large stoichiometric excess of acrylate or ketone were used in the coupling reaction, which in itself increases selectivity.

A possible mechanism for the 3,5-dicoupled cispentacin derivative is depicted in Figure 7. Because of the high concentration of methyl acrylate $\mathbf{1 4 5}$ in the first step, Ru-containing complex Ru-V was formed by ligand exchange (metathesis reaction) with pre-catalyst HG2. Next, [2+2] cycloaddition occurs between the newly-formed carbene $\mathbf{R u}-\mathbf{V}$ and 3,5-divinylated cispentacin derivatives, which leads to the corresponding metallacyclobutane intermediate Ru-VI. Formation of the monocoupled product and the Ru-containing methylidene complex Ru-I by redistribution of the electrons take place in the cycloreversion step. The other vinyl group on the monocoupled 5-membered cyclic $\beta$-amino ester can be functionalised again with a further methyl acrylate $\mathbf{1 4 5}$ (in carbene form $\mathbf{R u}-\mathbf{V}$ ) via metathesis reaction. First, cycloaddition occurs between newly formed mono-metathesised $\beta$-amino ester and $\mathbf{R u}-\mathbf{V}$ affording the corresponding metallacyclobutane Ru-VII. Formation of the desired dicoupled product takes place in the cycloreversion step by redistribution of the electrons. Finally, the reaction of the newly-formed Ru-based methylidene complex Ru-I with methyl acrylate $\mathbf{1 4 5}$ reproduces Ru-alkylidene $\mathbf{R u}-\mathbf{V}$ which starts the catalytic cycle from the beginning. 
Formation of $\mathbf{R u}-\mathbf{V}$ complex

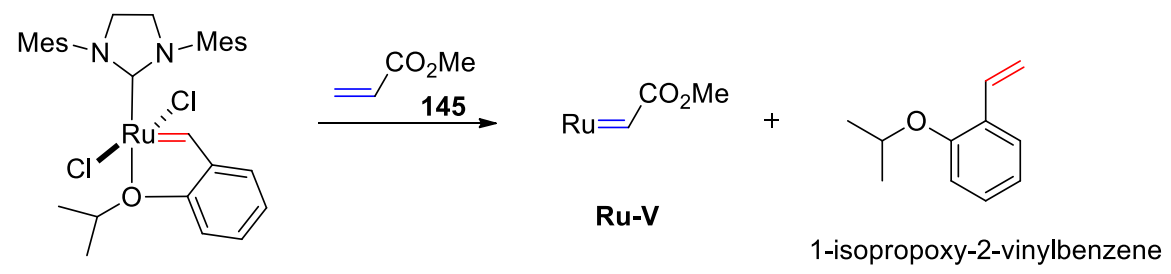

Hoveyda-Grubbs $2^{\text {nd }}$ pre-catalyst

Catalityc cycle of 3,5-divinylated cispentacin derivative

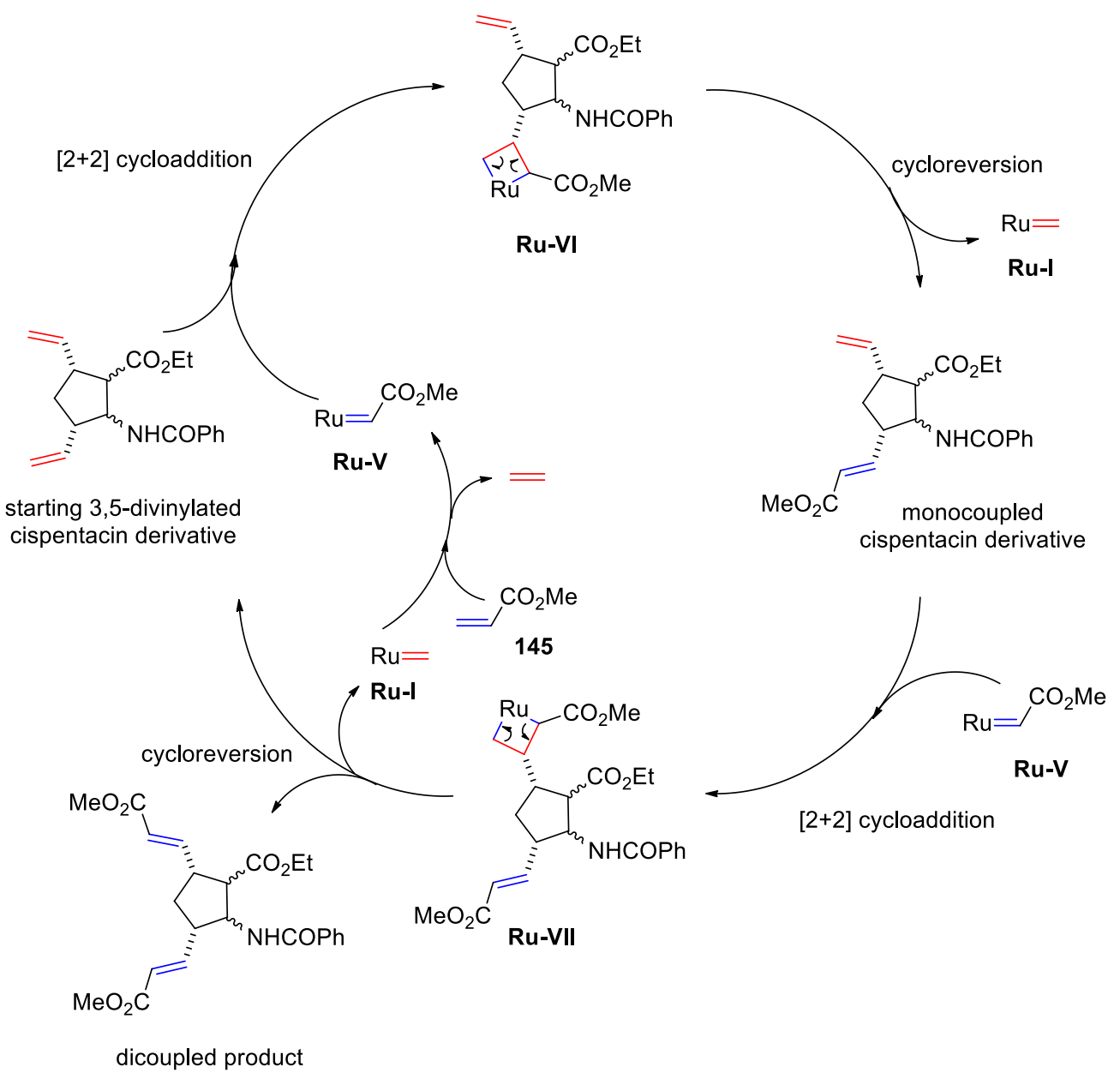

Figure 7. 


\subsection{Syntheses of functionalised $\beta$-amino acid derivatives and $\beta$-lactams through chemoselective cross metathesis $(\mathrm{CM})$}

Chemoselectivity was observed in the field of metathesis reactions (RCM, CM, and EYM) in several cases. It can originate from multiple factors, including the choice of catalyst, since the outcome of these reactions is often catalyst dependent. Steric or electronic deactivation of one of the $\mathrm{C}-\mathrm{C}$ double bonds is also capable of inducing chemoselectivity. ${ }^{120}$ Furthermore, several studies reported plausible hydrogen bond interaction in the pre-assembly phase between the catalyst halogen ligand and the substrate hydrogen atom, which favors the selective transformation of a certain $\mathrm{C}-\mathrm{C}$ double bond. ${ }^{143-145}$ It was also reported that the metallacyclobutane intermediate can be stabilised by interaction of the metal centre with nearby donor groups of the substrate, hindering further transformation of the involved $\mathrm{C}-\mathrm{C}$ double bond. ${ }^{146}$

During our experimental investigation we realised that under appropriately selected reaction conditions, chemodiscrimination of the olefinic bonds in cross metathesis reactions is achievable. Reactions of some divinylated $\beta$-amino esters and a divinylated $\beta$-lactam (derived from ROM reactions) with methyl vinyl ketone or acrylate esters in the presence of various metathesis catalysts afforded monocoupled products in a chemoselective manner. The aim of this section is the presentation of these reactions and to explain the reasons behind their chemoselectivity.

We have started our experiments with the investigation of cross metathesis reaction of divinyl-substituted azetidinone ( \pm )-126 derived from norbornene $\beta$-lactam $( \pm)$-105 by ROM reaction (Scheme 33). This bicyclic lactam was subjected to coupling reactions with methyl vinyl ketone 148 or acrylic esters 47 and 145 in the presence of commercially available G1, G2, HG1, and HG2 catalysts. However, coupling products $( \pm)-\mathbf{1 5 5}$ and $( \pm)-\mathbf{1 5 6} \mathbf{6}, \mathbf{b}$ were detected (by TLC monitoring) only in the presence of second generation catalysts. After trying several experimental conditions, the best yields were achieved by using 5 mol\% HG2 catalyst in dry $\mathrm{CH}_{2} \mathrm{Cl}_{2}$ for $4 \mathrm{~h}$. Monocoupled products, involving the $\alpha, \beta$-unsaturated carbonyl part located near to the amide $N$-atom, were isolated in moderate yields (see Table 6). It is important to note that our attempts to increase the yields of the mono-metathesised products failed because of various side reactions, such as polymerisation and formation of dicoupled products. 


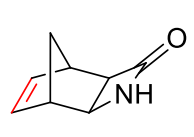

$( \pm)-105$

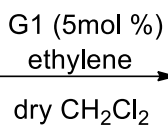

$20{ }^{\circ} \mathrm{C}, 3 \mathrm{~h}$

$80 \%$

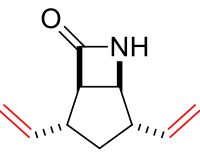

$( \pm)-126$

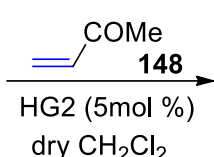

$20{ }^{\circ} \mathrm{C}, 4 \mathrm{~h}$

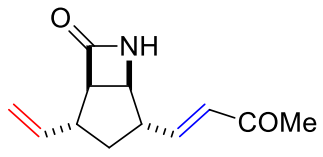

(士)-155

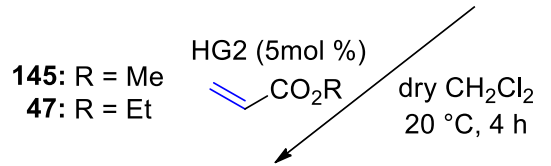

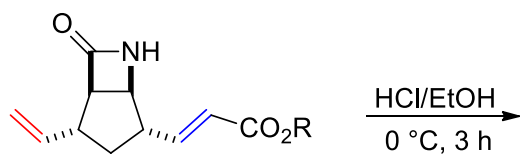

(士)-3

$( \pm)-156 \mathrm{a}: \mathrm{R}=\mathrm{Me}$

$( \pm)-156 b: R=E t$

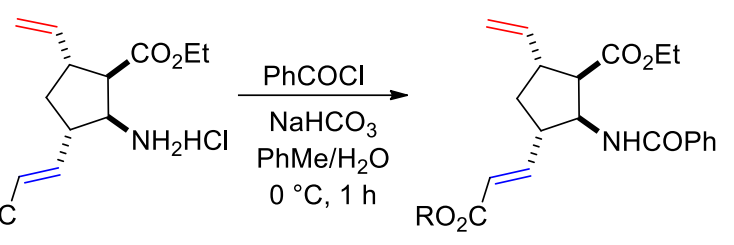

( \pm -157a: $R=\operatorname{Me}(58 \%)$

( \pm -157b: R = Et $(89 \%)$
$( \pm)-158 a: R=\operatorname{Me~}(60 \%)$

( \pm -158b: $R=$ Et $(77 \%)$

Scheme 33.

Table 6. Isolated yields for $( \pm)-\mathbf{1 5 5}$ and $( \pm)-\mathbf{1 5 6 a}, \mathbf{b}$ in selective CM reactions with HG2 catalyst.

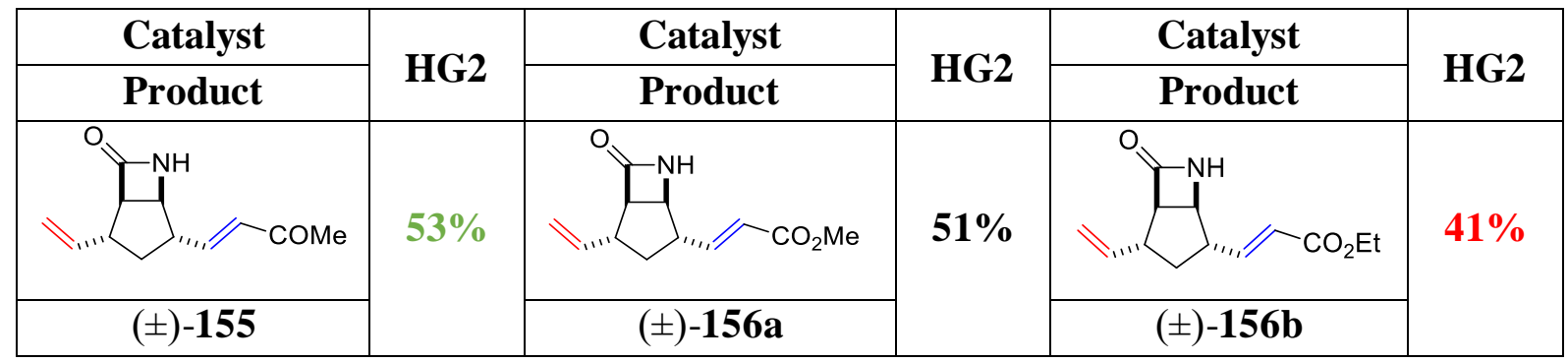

Monocoupled $\beta$-lactams $( \pm)$-156a,b were considered to be suitable precursors for stereocontorolled access to different cispentacin derivatives with $\mathrm{C}-\mathrm{C}$ double bond via opening of the heterocyclic ring. Thus, monocoupled cispentacin ester hydrochlorides $( \pm)-\mathbf{1 5 7} \mathbf{a}, \mathbf{b}$ were synthesised from the corresponding $\beta$-lactams $( \pm)-\mathbf{1 5 6 a}, \mathbf{b}$ by ethanolysis. Importantly, transesterification did not take place in the case of $( \pm)$-156a. Finally, the amino function was protected by benzoylation to access novel monocoupled cyclic $\beta$-amino esters $( \pm)-\mathbf{1 5 8 a}, \mathbf{b}$ (Scheme 33). Unfortunately, attempted ring-opening of compound ( \pm )-155 by $\mathrm{HCl} / \mathrm{EtOH}$ resulted in polymerisation.

Although less known in metal-catalysed processes, we assumed in our first approach that hydrogen bonding interaction between the halogen atom of the $\mathrm{Ru}$-alkylidene complex and the amide $\mathrm{N}-\mathrm{H}$ moiety as hydrogen bonding donor function could force the olefin bond closer to the amide $N$-atom to participate in the coupling reaction (Fig. 8). ${ }^{143-145}$ This leads to the mono-metathesised product before further $\mathrm{CM}$ reaction could occur. 


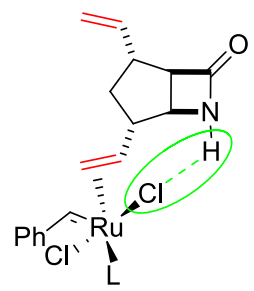

Figure 8.

In further studies, we were interested in the hydrogen bonding directing effect. To verify our hypothesis, $N$-Boc-protected divinylated $\beta$-lactam $( \pm)$-160 was prepared by ROM reaction with G1 catalyst under ethylene atmosphere from the corresponding tricyclic compound $( \pm)$-159 (Scheme 34). The resulting metathesis substrate $( \pm)-\mathbf{1 6 0}$, which cannot function as a hydrogen donor, was subjected to CM reaction with ethyl acrylate $\mathbf{4 7}$ in the presence of HG2 catalyst. According to our expectation, when the directing effect was excluded, the coupling reaction was not selective and afforded a mixture of the two mono-metathesised isomers $( \pm)$-161 and $( \pm)$-162 in a ratio close to 2:1 determined by the NMR analysis of the crude mixture. Unfortunately, our attempts to separate and isolate these two monocoupled $\beta$-lactams failed.

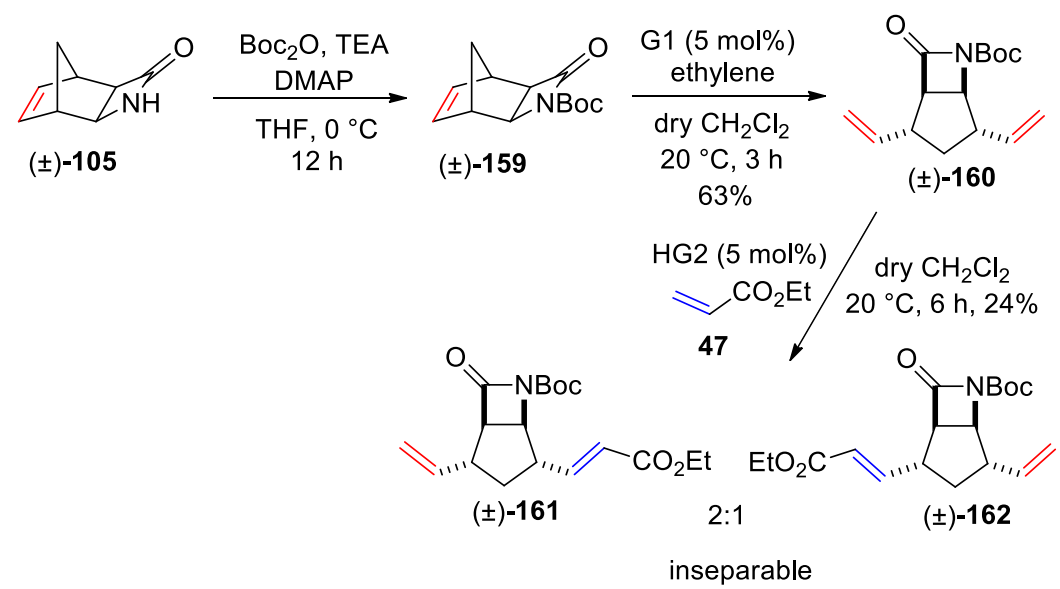

Scheme 34.

After successful chemoselective coupling of $( \pm)-\mathbf{1 2 6}$, we have expanded the scope of the metathesis substrates for these reactions. $\beta$-Amino esters with 5 -membered ring were used as our next starting compounds. First, divinyl-substitued cispentacin $( \pm)-\mathbf{1 1 5}$ (prepared by our ROM method) was submitted to CM reactions with acrylic esters 47 and 145 (Scheme 35). However, contrary to our previous experimental results, these cross metathesis reactions of compound $( \pm)$-115 in view of monocoupled products were not completely selective. As determined by ${ }^{1} \mathrm{H}-\mathrm{NMR}$ analysis, a mixture of two regioisomers $( \pm)-\mathbf{1 5 8}$ and $( \pm)-\mathbf{1 6 3}$ was formed in a 4:1 ratio through a partial hydrogen bonding directing effect. 


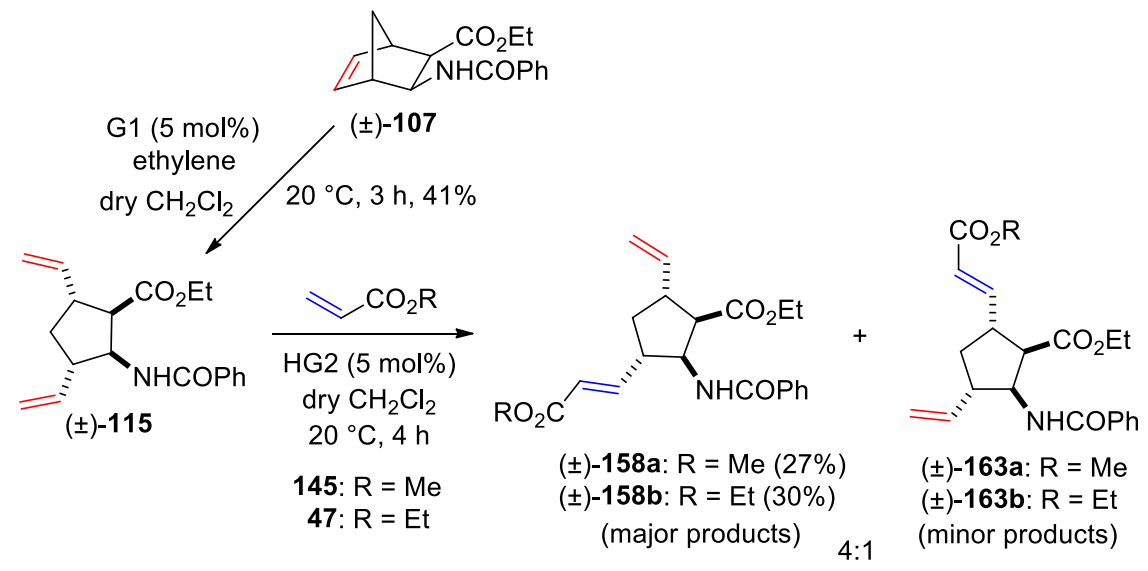

Scheme 35 .

After several failed attempts to separate the two regioisomers by column chromatography, the major products $( \pm)-\mathbf{1 5 8 a}, \mathbf{b}$ where the $\alpha, \beta$-unsaturated ester part is located near to the amide $\mathrm{N}$-atom could be successfully isolated by crystallisation from hexane/EtOAc. Their NMR data were completely identical with the final products shown in Scheme 33 thereby proving unambiguously their structure. Because of the limitations of crystallisation and metathesis side reactions such as the formation of dicoupled products or polymerised material, chemoselective coupling resulted in moderate but acceptable yields.

After the unexpected experimental results (two isomers instead of one), we next investigated the chemical properties of diolefinated transpentacin $( \pm)-\mathbf{1 1 7}$ in chemoselective $\mathrm{CM}$ reactions. For this purpose, divinylated amino ester $( \pm)-\mathbf{1 1 7}$, where the protected amino group and the ester moiety are in trans relationship, was prepared by the usual ROM protocol (Scheme 36).

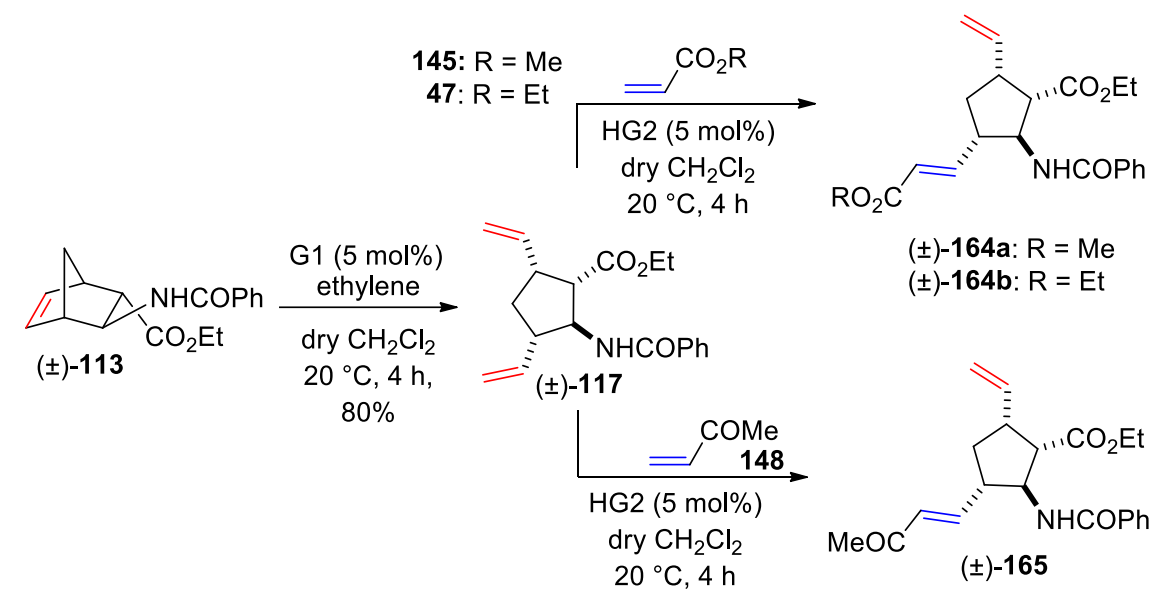

Scheme 36. 
Accordingly, compound ( \pm )-117 was submitted to cross-coupling with acrylic esters $\mathbf{4 7}$ and 145 or methyl vinyl ketone 148 at room temperature in dry $\mathrm{CH}_{2} \mathrm{Cl}_{2}$ with $\mathrm{HG} 2$ catalyst to afford the corresponding mono-metathesised products $( \pm)-\mathbf{1 6 4 a}, \mathbf{b}$ and $( \pm)-\mathbf{1 6 5}$ in medium yields (see Table 7). In contrast with divinylated cispentacin $( \pm)-\mathbf{1 1 5}$ [and at the same time similarly to divinylated $\beta$-lactam $( \pm)$-126], the CM reaction in these cases resulted in single monocoupled isomers. The structure of $\mathbf{1 6 4 a}$ was also determined by X-ray crystallography (Fig. 9).

Table 7. Isolated yields for $( \pm)-\mathbf{1 6 4 a}, \mathbf{b}$ and $( \pm)-\mathbf{1 6 5}$ in selective CM reactions with HG2 catalyst.

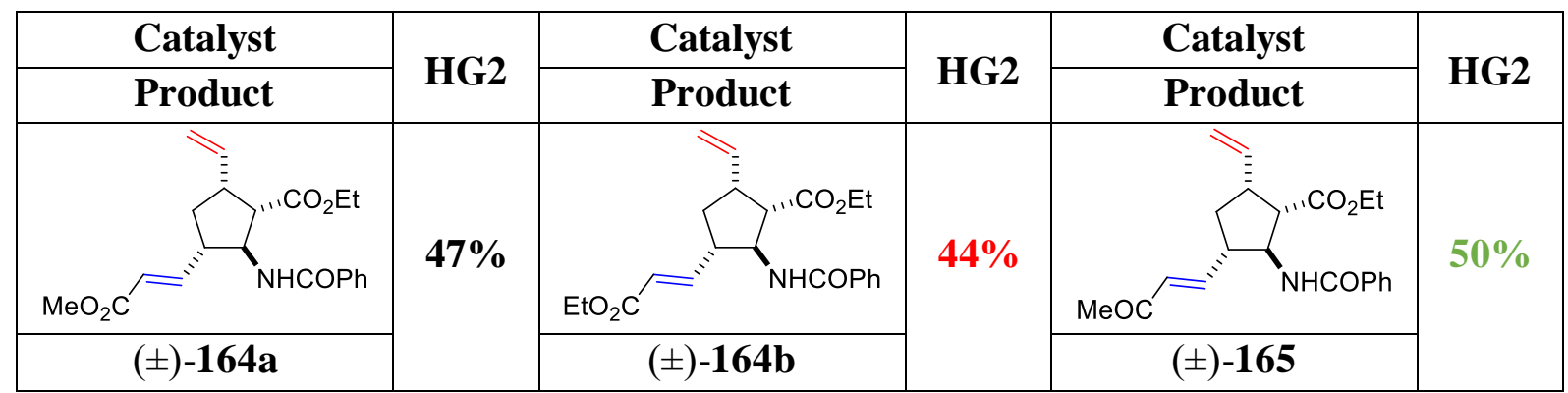

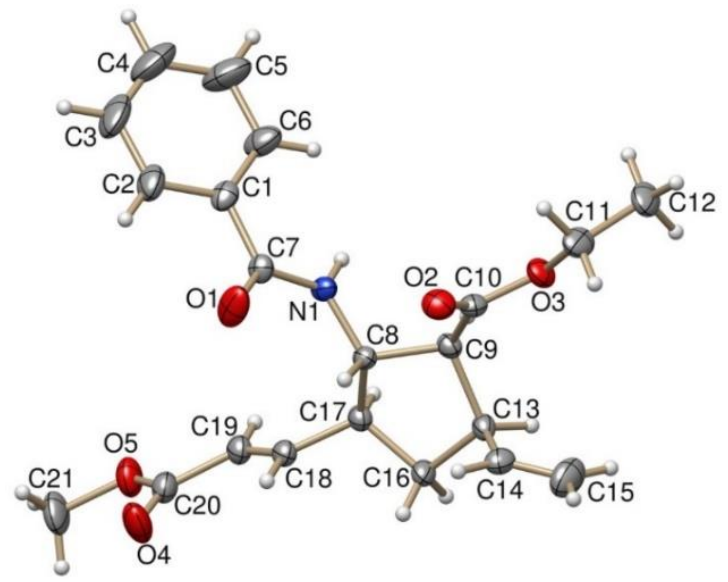

Figure 9. X-ray structure of compound 164a

The results of the NH-NBoc change found previously suggested that the reason of the observed chemoselectivity in CM reactions is a hydrogen bond between the chlorine atom of the catalyst and the $\mathrm{N}-\mathrm{H}$ moiety of the substrates. To further support this theory, we tried to disrupt this hydrogen bonding, which should decrease the selectivity. To achieve this goal, CM reaction between compound ( \pm )-117 and methyl acrylate $\mathbf{1 4 5}$, which previously afforded only a single isomer in $\mathrm{CH}_{2} \mathrm{Cl}_{2}$, was performed in three additional solvents (THF, dioxane, and toluene). The results were in complete agreement with our expectation and showed that solvents capable of participating in hydrogen bonding with the substrate (dioxane, THF) compete with the catalyst providing a mixture of regioisomers $( \pm)$-164a and $( \pm)-\mathbf{1 6 6}$ (approximately 2:1 ratio, 
determined by NMR analysis of the crude product). In contrast, solvents not being able to form a hydrogen bond $\left(\mathrm{CH}_{2} \mathrm{Cl}_{2}\right.$ and toluene) clearly gave only a single product (Schemes 36 and 37).

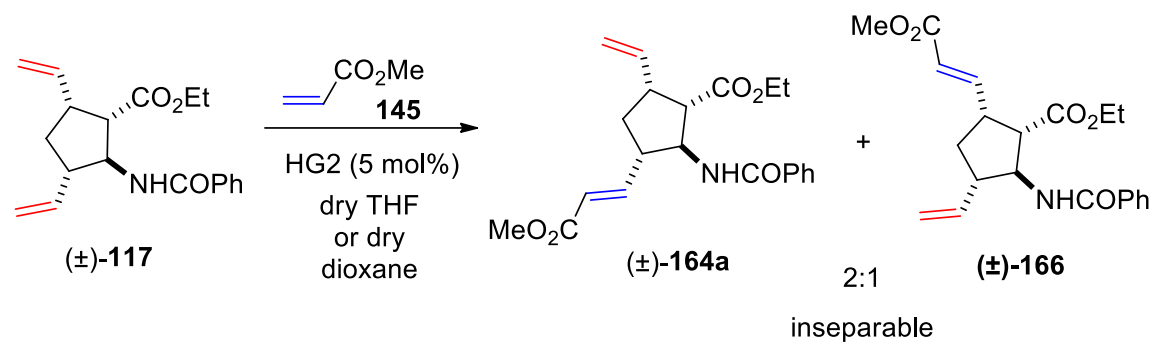

Scheme 37.

However, when another diolefinated transpentacin stereoisomer, namely $( \pm)$-118 (derived from diendo-norbornene $\beta$-amino ester $( \pm)$-112 by epimerisation and subsequent ROM) was submitted to $\mathrm{CM}$ reaction with $\alpha, \beta$-unsaturated carbonyl compounds 145 and $\mathbf{1 4 8}$, inseparable mixtures of regioisomers $( \pm)-\mathbf{1 6 7 / (}( \pm)-\mathbf{1 6 8}$ and $( \pm)-\mathbf{1 6 9} /( \pm)-\mathbf{1 7 0}$, respectively, were formed. Regioisomeric ratios, determined by NMR analysis of the crude products, were nearly 2:1 in both cases (Scheme 38).

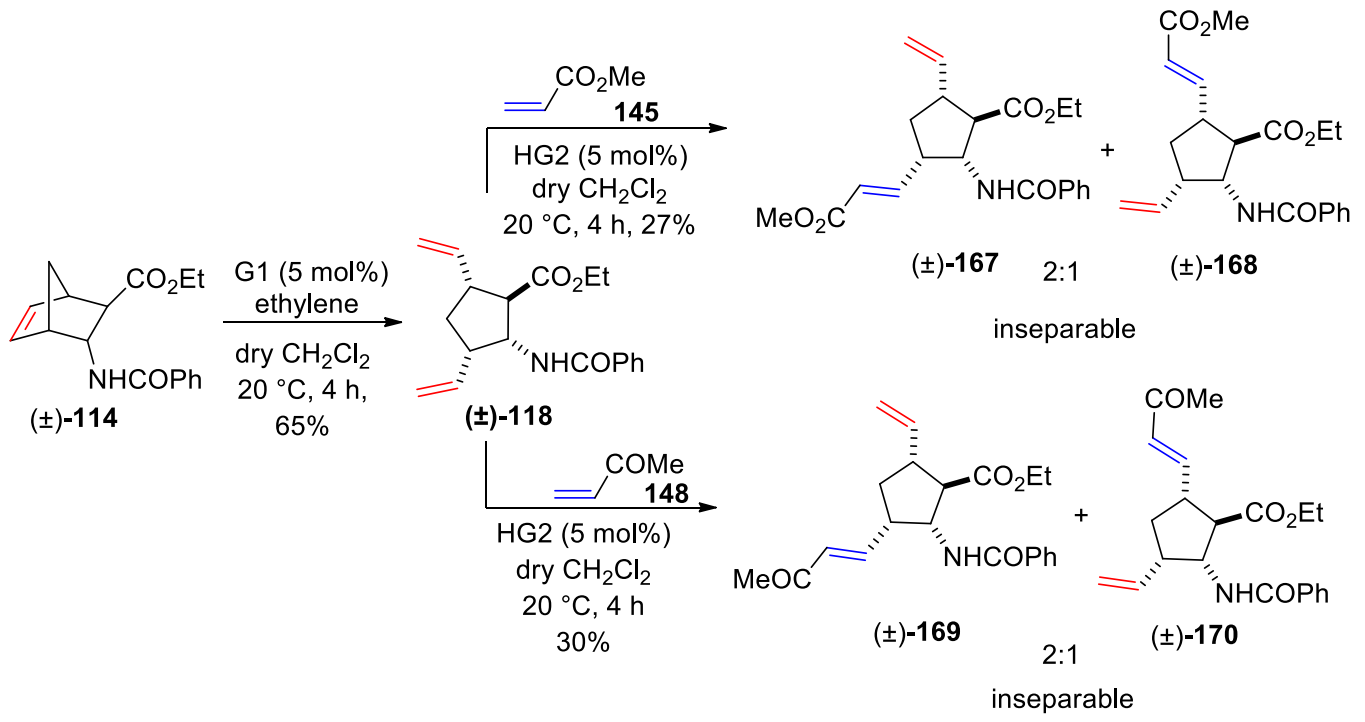

Scheme 38.

These results clearly suggested that in addition to the hydrogen bonding effect, other factors also influence the outcome of the coupling reactions. Chemoselectivity may originate from steric effects. It is also highly probable, that coordination of ruthenium to the carbonyl oxygen, which creates a stable 6-membered chelate ring (T1), stabilizes the metallacyclobutane and hinders further transformations. In the case of $\mathbf{T} 2$ and $\mathbf{T 3}$ structures, in which the vinyl side-chain and the ester function are trans to each other, formation of the chelate ring is less favored and, therefore, the cross metathesis reaction is not selective (Fig. 10). 


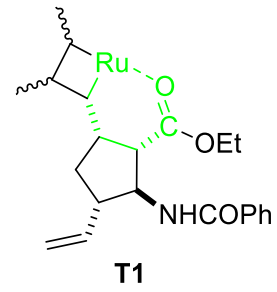

more stable 6-membered chelate ring

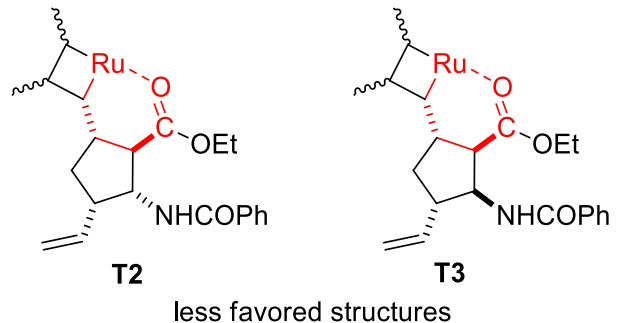

less favored structures

Figure 10. Chelate ring stability in the case of transpentacin derivatives $( \pm)-\mathbf{1 1 7},( \pm)-\mathbf{1 1 8}$, and $( \pm)-\mathbf{1 1 5}$.

Because of the importance of oxygen-containing heterocyclic $\beta$-amino acids (such as oxetin) we extended the scope of the chemoselective transformations with CM reactions to the synthesis of monocoupled $O$-heterocyclic counterparts. For this reason, diolefinated amino ester $( \pm)-\mathbf{1 2 4}$, in which the ester and the protected amino group are in a cis arrangement, was prepared by ROM reaction from the corresponding bicyclic starting material $( \pm)-\mathbf{1 2 2}$. Cross metathesis reaction was performed between acrylate ester 47 and compound $( \pm)-\mathbf{1 2 4}$ in the presence of HG2 catalyst. Similar to its carbocyclic counterpart $( \pm)-\mathbf{1 1 5}$, it afforded an inseparable mixture of $( \pm)$-171 and $( \pm)$-172 in nearly 2:1 ratio (Scheme 39).

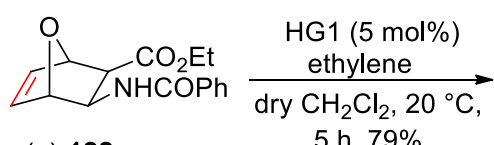

$( \pm)-122$

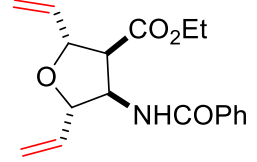

$( \pm)-124$
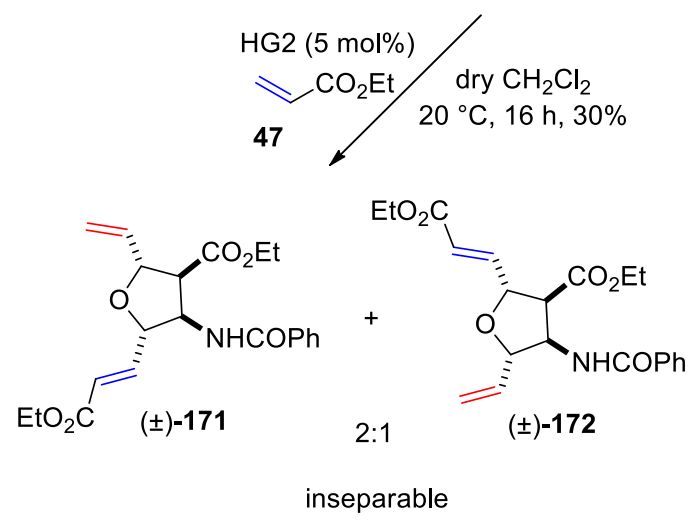

Scheme 39. 
Finally, it seemed to be logical to prepare the trans isomer of oxygen-containing heterocyclic ( \pm )-125 and subject it to CM reaction with ethyl acrylate 47 in the presence of HG2 catalyst (Scheme 40). For this purpose, oxanorbornene amino ester $( \pm)-\mathbf{1 2 2}$ was epimerised with $\mathrm{NaOEt}$ in $\mathrm{EtOH}$ at $20^{\circ} \mathrm{C}$ in moderate yield to afford metathesis substrate $( \pm)-\mathbf{1 2 3}$, which then underwent ROM reaction. According to our expectation (and confirming the chelate theory in these cases), the $\mathrm{CM}$ reaction led to single monocoupled product $( \pm)-\mathbf{1 7 3}$ analogously to trans amino ester $( \pm)-\mathbf{1 1 7}$.

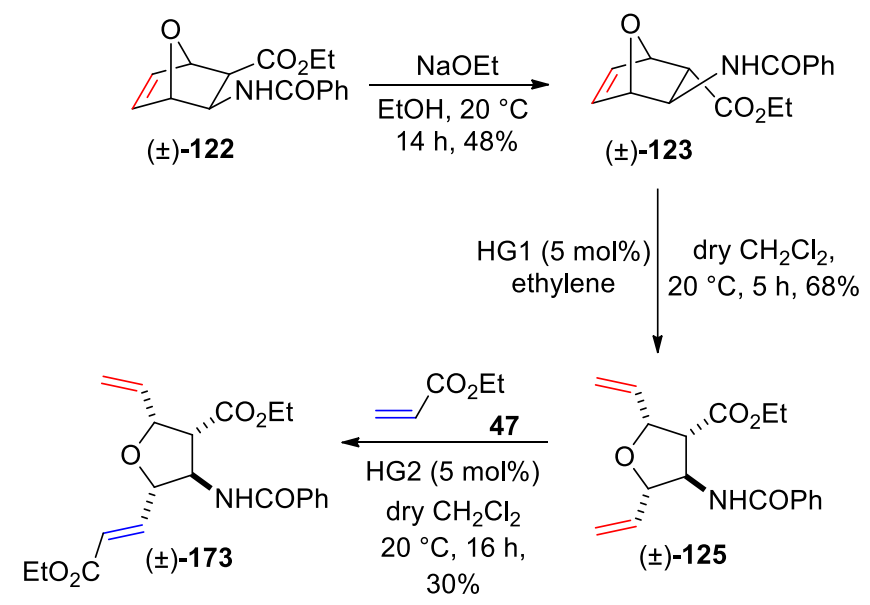

Scheme 40. 


\section{SUMMARY}

- An efficient, stereocontrolled one-step protocol has been developed for the syntheses of divinylated cispentacins 115, 116, 117, and 118 as well as oxacispentacin 124 and 125 stereoisomers with multiple chiral centres from diexo-norbornene 107 and diendo-norbornene 112 or oxanorbornene $\beta$-amino acid derivatives 122 by ROM reaction (Schemes 22 and 24).

- During ROM reactions, the stereocentres of the starting materials were unaffected allowing the transfer of chiral information to the final products. ROM reactions were executed in the presence of commercially available Ru-based first and second generation Grubbs and Hoveyda-Grubbs catalysts (G1, G2, HG1, and HG2) with ethylene under argon atmosphere.

- In the case of cispentacin derivatives $\mathbf{1 1 5}$ and 116, clear correlation between yields, the used catalysts and the structure of starting materials 107 and 112 was not observed, although HG2 catalyst was slightly more effective than the others. However, when the carboxyl and the amino function were in trans arrangement in starting materials 113 and 114, first generation catalysts G1 and HG1 gave yields more than two times higher. Lower yields found with diexo 107 and diendo 112 derivatives may be explained by chelation of the catalyst.

- The well-established one-step synthetic protocol was also applied for the access of optically pure divinylated cispentacin derivatives (+)-115 and (-)-117 from an enantiomerically enriched diexo-norbornene $\beta$-lactam (+)-105 obtained by enzymatic kinetic resolution of racemic azetidinone 105 (Scheme 23).

- The stereocontrolled ring-opening method was applied to the preparation of a divinylated bicyclic $\beta$-lactam 126 and monocyclic $\beta$-lactams 131 and 136 with terminal alkene functions. The ROM reactions in all cases $[\beta$-lactams and (oxa)norbornene $\beta$-amino acid derivatives] were largely facilitated by the high ring strain of the unsaturated starting materials, which is released during these transformations.

- Functionalised $\beta$-lactams 126, 131, and 136 were suitable precursors for stereocontrolled access of divinylated cispentacins or open-chain $\beta$-amino acid derivatives with anti arrangement of the carboxyl and amino moieties. Thus, these valuable olefinated $\beta$-amino acids 127,132 , and 137 or esters 128,133 , and 138 were prepared through the opening of the 4-membered heterocyclic ring by aqueous or ethanolic solution of $\mathrm{HCl}$ (Schemes 25, 26, and 27). 
- Ring-opened products containing the valuable olefin bond were further functionalised with $\alpha, \beta$-unsaturated carbonyl compounds (methyl acrylate 145 or methyl vinyl ketone 148 ) through CM reaction (Schemes 29, 30, 31, and 32). The coupling reactions were executed in the presence of HG2 catalyst affording dicoupled azetidinones 149, 150, 151, 152, 153 and 154 as well as dicoupled $\beta$-amino acid derivatives 146 and 147 with $E$ geometry with moderate to good yields (see Tables 3, 4, and 5).

- In the final section, olefin chemodifferentiation of divinylated products $115,117,118$, 124, 125, 126, and 160 (derived from $\mathrm{ROM}$ ) through $\mathrm{CM}$ reactions was presented. With a simple modification of reaction conditions, valuable monocoupled azetidinones $\mathbf{1 5 5}$ and 156a,b, cispentacin 158a,b, 164a,b, and 165 as well as oxacispentacin derivatives 173 were the main products instead of previously described disubstituted products (Schemes 33, 35, 36, and 40).

- The selectivity in the case of bicyclic $\beta$-lactam 126 originates from the hydrogen bond between the catalyst chlorine atom and the $\mathrm{N}-\mathrm{H}$ moiety, which was confirmed by $\mathrm{NH}-\mathrm{NBoc}$ exchange (Figure 8 and Scheme 34). However, in the case of 5-membered cyclic $\beta$-amino acid derivatives 115, 117, and 118, selectivity was determined by multiple factors (Schemes 35, 36, 37, and 38).

- Besides of secondary interactions, chelate ring stability also proved to be responsible for chemodifferentiation (Figure 10). This resulted in a single mono-metathesised product in the cases of transpentacin 117 and its oxa-analogue 125. In contrast, a mixture of regioisomers were formed when cispentacin 115, its oxa-analogue 124 and transpentacin 118 were used as starting material (Schemes 35, 36, 38, 39, and 40). The structure of $\mathbf{1 6 4 a}$ was also certified by X-ray crystallography (Figure 9). 


\section{ACKNOWLEDGEMENTS}

The presented work was carried out in the Institute of Pharmaceutical Chemistry, University of Szeged between 2013 and 2016.

I would like to express my gratitude to my supervisors Professor Loránd Kiss head of the Institute of Pharmaceutical Chemistry and Professor Ferenc Fülöp for their scientific guidance, their inspiring ideas, and their constructive criticism to complete this work.

I am grateful to Professor Enikő Forró for the preparation of enantiomerically pure $\beta$-lactam as starting material and for the GC and HPLC measurements.

I owe my thanks to Professor Árpád Molnár for revising the English of my thesis.

I would like to thank all my colleagues for their help, friendship and the pleasant working atmosphere, and especially my colleagues from the $2^{\text {nd }}$ laboratory, Dr. Melinda Nonn, Attila Márió Remete, dr. Renáta Anita Ábrahámi, and Kitti Vasvári.

Finally, I wish to express my warmest thanks to my family and friends for their encouragement and understanding during my $\mathrm{PhD}$ studies. 


\section{REFERENCES}

1. Fülöp, F. Chem. Rev. 2001, 101, 2181.

2. Kiss, L.; Fülöp, F. Chem. Rev. 2014, 114, 1116.

3. "Synthesis of Carbocyclic $\beta$-Amino Acids": Kiss. L.; Forró. E.; Fülöp. F. in Amino Acids, Peptides and Proteins in Organic Chemistry, Vol. 1 (Ed.: Hughes, A. B.) Wiley, Weiheim 2009, p. 367.

4. Kuhl, A.; Hahn, M. G.; Dumic, M.; Mittendorf, J. Amino Acids 2005, 29, 89.

5. Park, K. H.; Kurth, M. J. Tetrahedron 2002, 58, 8629.

6. Pandey, S. K.; Jogdand, G. F.; Oliveira, J. C. A.; Mata, R. A.; Rajamohanan, P. R.; Ramana, C. V. Chem. Eur. J. 2011, 17, 12946.

7. Coursindel, T.; Martinez, J.; Parrot, I. Eur. J. Org. Chem. 2011, 4519.

8. Chandrasekhar, S.; Sudhakar, A.; Kiran, M. U.; Babu, B. N.; Jagadeesh, B. Tetrahedron Lett. 2008, 49, 7368 .

9. Mittendorf, J.; Kunisch, F.; Matzke, M.; Militzer, H-C.; Schmidt, A.; Schönfeld, W. Bioorg. Med. Chem. Lett. 2003, 13, 433.

10. Forró, E.; Fülöp, F. Mini Rev. Org. Chem. 2004, 1, 93.

11. Hook, D. F.; Bindschädler, P.; Mahajan, Y. R.; Šebesta, R.; Kast, P.; Seebach, D. Chem. Biodivers. 2005, 2, 591.

12. Haase, H. S.; Peterson-Kaufman, K. J.; Levengood, S. K. L.; Checco, J. W.; Murphy, W. L.; Gellman, S. H. J. Am. Chem. Soc. 2012, 134, 7652.

13. Lelais, G.; Seebach, D. Biopolymers 2004, 76, 206.

14. Porter, E. A.; Weisblum, B.; Gellman, S. H. J. Am. Chem. Soc, 2005, 127, 11516.

15. Martinek, T. A.; Fülöp, F. Chem. Soc. Rev. 2012, 41, 687.

16. Berlicki, L.; Pilsl, L.; Wéber, E.; Mándity, I. M.; Cabrele, C.; Martinek, T. A.; Fülöp, F.; Reiser, O.; Angew. Chem. Int. Ed. 2012, 51, 2208.

17. Connon, S. J.; Blechert, S.; Angew. Chem. Int. Ed. 2003, 42, 1900.

18. Monsaert, S.; Vila, A. L.; Drozdzak, R.; Van Der Voort, P.; Verpoort, F. Chem. Soc. Rev. 2009, 38, 3360 .

19. Imhof, S.;. Randl, S.; Blechert.; S. Chem. Commun. 2001, 1692.

20. Kress, S.; Blechert, S. Chem. Soc. Rev. 2012, 41, 4389.

21. Koh, M. J.; Nguyen, T. T.; Lam, J. K.; Torker, S.; Hyvl, J.; Schrock, R. R.; Hoveyda, A. H. Nature, 2017, 542, 80.

22. Chauvin, Y. Angew. Chem. Int. Ed. 2006, 45, 3741. 
23. Sanford, M. S.; Love, J. A.; Grubbs, R. H. J. Am. Chem. Soc. 2001, 123, 6543.

24. Schrodi, Y.; Pederson, R. L. Aldrichimica Acta 2007, 40, 45.

25. Smith, B. J.; Sulikowski, G. A. Angew. Chem. Int. Ed. 2010, 49, 1599.

26. Prunet, J. Eur. J. Org. Chem. 2011, 3634.

27. Van de Wegh, P.; Eustache, J. Curr. Top. Med. Chem. 2005, 5, 1495.

28. Felpin, F-X.; Lebreton, J. Eur. J. Org. Chem. 2003, 3693.

29. Jakubec, P.; Cockfield, D. M.; Dixon, D. J. J. Am. Chem. Soc. 2009, 131, 16632.

30. Rosillo, M.; Domínguez, G.; Casarrubios, L.; Amador, U.; Pérez-Castells, J. J. Org. Chem. 2004, 69, 2084.

31. Meek, S. J.; O’Brien, R. V.; Llaveria, J.; Schrock, R. R.; Hoveyda, A. H. Nature, 2011, $471,461$.

32. Cortez, G. A.; Baxter, C. A.; Schrock, R. R.; Hoveyda, A. H. Org. Lett. 2007, 9, 2871

33. Mol, J. C. Catal. Today 1999, 51, 289.

34. Mol, J. C. J. Mol. Catal. A: Chem. 2004, 213, 39.

35. Mol, J. C. Green Chem. 2002, 4, 5.

36. Chikkali, S.; Mecking, S. Angew. Chem. Int. Ed. 2012, 51, 5802.

37. Higman, C. S.; Lummiss, J. A. M.; Fogg, D. E. Angew. Chem. Int. Ed. 2016, 55, 3552.

38. Mol, J. C. Top. Catal. 2004, 27, 97.

39. Rybak, A.; Fokou, P. A.; Meier, M. A. R. Eur. J. Lipid Sci. Technol. 2008, 110, 797.

40. Nonn, M.; Kiss, L.; Forró, E.; Sillanpää, R.; Fülöp, F. Beilstein J. Org. Chem. 2012, 8, 100.

41. Nonn, M.; Kiss, L.; Hänninen, M. M.; Sillanpää, R.; Fülöp, F. Chem. Biodivers. 2012, 9, 2571.

42. Kiss, L.; Nonn, M.; Sillanpää, R.; Fustero, S.; Fülöp, F. Beilstein J. Org. Chem. 2013, 9,1164 .

43. Kiss, L.; Forró, E.; Orsy, G.; Ábrahámi, R.; Fülöp, F. Molecules 2015, 20, 21094.

44. Kiss, L.; Szatmári, I.; Fülöp, F. Lett. Org. Chem. 2006, 3, 463.

45. Kiss, L.; Forró, E.; Sillanpää, R.; Fülöp, F. J. Org. Chem. 2007, 72, 8786.

46. Kazi, B.; Kiss, L.; Forró, E.; Mándity, I.; Fülöp, F. Arkivoc 2010, ix, 31.

47. Kiss, L.; Forró, E.; Sillanpää, R.; Fülöp, F. Tetrahedron 2010, 66, 3599.

48. Kiss, L.; Forró, E.; Sillanpää, R.; Fülöp, F. Synthesis 2010, 153.

49. Kiss, L.; Forró, E.; Sillanpää, R.; Fülöp, F. Nucleic Acids Symp. Ser. 2008, 52, 551.

50. Kiss, L.; Forró, E.; Sillanpää, R.; Fülöp, F. Tetrahedron: Asymmetry 2008, 19, 2856. 
51. Kiss, L.; Forró, E.; Martinek, T. A.; Bernáth, G.; De Kimpe, N.; Fülöp, F. Tetrahedron 2008, 64, 5036.

52. Kiss, L.; Forró, E.; Fustero, S.; Fülöp, F. Eur. J. Org. Chem. 2011, 4993.

53. Kiss, L.; Forró, E.; Fustero, S.; Fülöp, F. Org. Biomol. Chem. 2011, 9, 6528.

54. Kiss, L.; Kazi, B.; Forró, E.; Fülöp, F. Tetrahedron Lett. 2008, 49, 339.

55. Kiss, L.; Cherepanova, M.; Forró, E.; Fülöp, F. Chem. Eur. J. 2013, 19, 2102.

56. Cherepanova, M.; Kiss, L.; Sillanpää, R.; Fülöp, F. RSC Adv. 2013, 3, 9757.

57. Cherepanova, M.; Kiss, L.; Forró, E.; Fülöp, F. Eur. J. Org. Chem. 2014, 403.

58. Kiss, L.; Nonn, M.; Forró, E.; Sillanpää, R.; Fustero, S.; Fülöp, F. Eur. J. Org. Chem. 2014, 4070 .

59. Nonn, M.; Kiss, L.; Haukka, M.; Fustero, S.; Fülöp, F. Org. Lett. 2015, 17, 1074.

60. Kiss, L.; Remete, A. M.; Nonn, M.; Fustero, S.; Sillanpää, R.; Fülöp, F. Tetrahedron 2016, 72,781 .

61. Kiss, L.; Nonn, M.; Sillanpää, R.; Haukka, M.; Fustero, S.; Fülöp, F. Chem. Asian. J. 2016, 11, 3376.

62. Ábrahámi, R. A.; Kiss, L.; Barrio, P.; Fülöp, F. Tetrahedron 2016, 72, 7526.

63. Remete, A. M.; Nonn, M.; Fustero, S.; Fülöp, F.; Kiss, L. Molecules 2016, 21, 1493.

64. Chauvin, Y.; Hérisson, J.-L. Makromol. Chem. 1971, 141, 161.

65. Calderon. N. Tetrahedron Lett. 1967, 34, 3327.

66. Astruc, D. New J. Chem. 2005, 29, 42.

67. Trnka, T. M.; Grubbs, R. H. Acc. Chem. Res. 2001, 34, 18.

68. Brik, A. Adv. Synth. Catal. 2008, 350, 1661.

69. Formentín, P.; Gimeno, N.; Steinke, J. H. G.; Vilar, R. J. Org. Chem. 2005, 70, 8235.

70. Fürstner, A. Angew. Chem. Int. Ed. 2000, 39, 3012.

71. Grubbs, R. H.; Chang, S. Tetrahedron 1998, 54, 4413.

72. Hamad, F. B.; Sun, T.; Xiao, S.; Verpoort, F. Coord. Chem. Rev. 2013, 257, 2274.

73. Donohoe, T. J.; Basutto, J. A.; Bower, J. F.; Rathi, A. Org. Lett. 2011, 13, 1036.

74. Schuster, M.; Blechert, S. Angew. Chem. Int. Ed. Engl. 1997, 36, 2036.

75. Vougioukalakis, G. C.; Grubbs, R. H. Chem. Rev. 2010, 110, 1746.

76. Schneider, V.; Frolich P. K. Ind. Eng. Chem. 1931, 23, 1405.

77. Eleuterio, H. S. German Pat. 1960, 1072811

78. Banks, R. L.; Bailey, G. C. Ind. Eng. Chem. Prod. Res. Dev. 1964, 3, 170.

79. Rouhi, A. M. Chem. Eng. News 2002, 80, 34.

80. Grubbs, R. H. Angew. Chem. Int. Ed. 2006, 45, 3760. 
81. Truett, W. L.; Johnson, D. R.; Robinson, I. M.; Montague, B. A. J. Am. Chem. Soc. 1960, 82, 2337.

82. Natta, G.; Dall'asta, G.; Mazzanti, G. Angew. Chem. Int. Ed. Engl. 1964, 3, 723.

83. Nguyen, S. T.; Johnson, L. K.; Grubbs, R. H. J. Am. Chem. Soc. 1992, 114, 3974.

84. Bradshaw, C. P. C.; Howman, E. J.; Turner, L. J. Catal. 1967, 7, 269.

85. Calderon, N.; Chen, H. Y.; Scott, K. W. Tetrahedron Lett. 1967, 3327.

86. Calderon, N.; Ofstead, E. A.; Ward, J. P.; Judy, W. A.; Scott, K. W. J. Am. Chem. Soc. 1968, 90, 4133.

87. Grubbs, R. H.; Brunck, T. K. J. Am. Chem. Soc. 1972, 94, 2538.

88. Grubbs, R. H.; Burk, P. L.; Carr, D. D. J. Am. Chem. Soc. 1975, 97, 3265.

89. Grubbs, R. H.; Carr, D. D.; Hoppin, C.; Burk, P. L. J. Am. Chem. Soc. 1976, 98, 3478.

90. Katz, T. J.; McGinnis, J. J. Am. Chem. Soc. 1975, 97, 1592.

91. Katz, T. J.; Rothchild, R. J. Am. Chem. Soc. 1976, 98, 2519.

92. Katz, T. J.; McGinnis, J. J. Am. Chem. Soc. 1977, 99, 1903.

93. Lee, S. J.; McGinnis, J.; Katz, T. J. J. Am. Chem. Soc. 1976, 98, 7818.

94. McGinnis, J.; Katz, T. J.; Hurwitz, S. J. Am. Chem. Soc. 1976, 98, 605.

95. Howard, T. R.; Lee, J. B.; Grubbs, R. H. J. Am. Chem. Soc. 1980, 102, 6876.

96. Wengrovius, J. H.; Sancho, J.; Schrock, R. R. J. Am. Chem. Soc. 1981, 103, 3932.

97. Mol, J. C.; Moulijn, J. A.; Boelhouwer, C. Chem. Commun. 1968, 633.

98. Woodward, R. B.; Hoffmann, R. J. Am. Chem. Soc. 1965, 87, 395.

99. http://www.nobelprize.org/nobel_prizes/chemistry/laureates/2005/animation.html

100. Schrock, R. R. Chem. Rev. 2009, 109, 3211.

101. Schrock, R. R. Chimia 2015, 69, 388.

102. Schrock, R. R.; Hoveyda, A. H. Angew. Chem. Int. Ed. 2003, 42, 4592.

103. Diesendruck, C. E.; Tzur, E.; Lemcoff, N. G. Eur. J. Inorg. Chem. 2009, 4185.

104. Bieniek, M.; Michrowska, A.; Usanov, D. L.; Grela, K. Chem. Eur. J. 2008, 14, 806.

105. Schwab, P.; France, M. B.; Ziller, J. W.; Grubbs, R. H. Angew. Chem. Int. Ed. Engl. 1995, 34, 2039.

106. Scholl, M.; Ding, S.; Lee, C. W.; Grubbs, R. H. Org. Lett. 1999, 1, 953.

107. Kingsbury, J. S.; Harrity, J. P. A.; Bonitatebus, P. J.; Hoveyda, A. H. J. Am. Chem. Soc. 1999, 121, 791.

108. Michrowska, A.; Bujok, R.; Harutyunyan, S.; Sashuk, V.; Dolgonos, G.; Grela, K. J. Am. Chem. Soc. 2004, 126, 9318.

109. Wakamatsu, H.; Blechert, S. Angew. Chem. Int. Ed. 2002, 41, 794. 
110. Barbasiewicz, M.; Bieniek, M.; Michrowska, A.; Szadkowska, A.; Makal, A.; Woźniak, K.; Grela, K. Adv. Synth. Catal. 2007, 349, 193.

111. Grela, K.; Harutyunyan, S.; Michrowska, A. Angew. Chem. Int. Ed. 2002, 41, 4038.

112. Wakamatsu, H.; Blechert, S. Angew. Chem. Int. Ed. 2002, 41, 2403.

113. Bates, J. M.; Lummiss, J. A. M.; Bailey, G. A.; Fogg, D. E. ACS Catal. 2014, 4, 2387.

114. Keim, W. Angew. Chem. Int. Ed. 2013, 52, 12492.

115. Kotha, S.; Meshram, M.; Khedkar, P.; Banerjee, S.; Deodhar, D. Beilstein J. Org. Chem. 2015, 11, 1833.

116. Holub, N.; Blechert, S. Chem. Asian J. 2007, 2, 1064.

117. Garcia-Fandiño, R.; Codesido, E. M.; Sobarzo-Sánchez, E.; Castedo, L.; Granja, J. R. Org. Lett. 2004, 6, 193.

118. Yang, Z.; He, Y.; Vourloumis, D.; Vallberg, H.; Nicolaou, K. C. Angew. Chem. Int. Ed. Engl. 1997, 36, 166.

119. Yee, N. K.; Farina, V.; Houpis, I. N.; Haddad, N.; Frutos, R. P.; Gallou, F.; Wang, X-J; Wei, X.; Simpson, R. D.; Feng, X.; Fuchs, V.; Xu, Y.; Tan, J.; Zhang, L.; Xu, J.; Smith-Keenan, L. L.; Vitous, J.; Ridges, M. D.; Spinelli, E. M.; Johnson, M. J. Org. Chem. 2006, 71, 7133 .

120. Nolan, S. P.; Clavier, H. Chem. Soc. Rev. 2010, 39, 3305.

121. Hoveyda, A. H; Zhugralin, A. R. Nature 2007, 450, 243.

122. Maier, M. E. Angew. Chem. Int. Ed. 2000, 39, 2073.

123. Gradillas, A.; Pérez-Castells, J. Angew. Chem. Int. Ed. 2006, 45, 6086.

124. ’Ring-Closing Metathesis": Van Lierop, B. J.; Lummiss, J. A. M.; Fogg, D. E. in Olefin Metathesis Theory and Practice (Ed.: Grela, K.) John Wiley \& Sons 2014, p. 85.

125. Kamau, S. D.; Hodge, P.; Hall, A. J.; Dad, S.; Ben-Haida, A. Polymer 2007, 48, 6808.

126. Nelson, D. J.; Ashworth, I. W.; Hillier, I. H.; Kyne, S. H.; Pandian, S.; Parkinson, J. A.; Percy, J. M.; Rinaudo, G.; Vincent, M. A. Chem. Eur. J. 2011, 17, 13087.

127. Abell, A. D.; Gardiner, J. Org. Lett. 2002, 4, 3663.

128. Gardiner J.; Anderson, K. H.; Downard, A.; Abell, A. D. J. Org. Chem. 2004, 69, 3375 .

129. Chippindale A. M.; Davies, S. G.; Iwamoto, K.; Parkin, R. M.; Smethurst, C. A. P.; Smith, A. D.; Rodriguez-Solla, H. Tetrahedron 2003, 59, 3253.

130. Davis, F. A.; Theddu, N. J. Org. Chem. 2010, 75, 3814.

131. Perlmutter, P.; Rose, M.; Vounatsos, F. Eur. J. Org. Chem. 2003, 756. 
132. "The nucleophilic addition/ring closure (NARC) sequence for the stereocontrolled synthesis of heterocycles": Perlmutter, P. in: Stereoselective Heterocyclic Synthesis II Vol. 190 (Ed.: Metz, P.) Topics in Current Chemistry 1997, p. 87.

133. Aparici, I.; Guerola, M. Dialer, C.; Simón-Fuentes, A.; Sánchez-Roselló, M.; del Pozo, C.; Fustero, S. Org. Lett. 2015, 17, 5412.

134. Fustero, S.; Sánchez-Roselló, M.; Sanz-Cervera, J. F.; Aceña, J. L.; del Pozo, C.; Fernández B.; Bartolomé, A.; Asensio, A. Org. Lett. 2006, 8, 4633.

135. Yudin, A. K. Chem. Sci. 2015, 6, 30.

136. Fustero, S.; Bartolomé, A.; Sanz-Cervera, J. F.; Sánchez-Roselló, M.; Soler, J. G.; de Arellano C. R.; Fuentes, A. S. Org. Lett. 2003, 5, 2523.

137. Yamanaka, T.; Ohkubo, M.; Kato, M.; Kawamura, Y.; Nishi, A.; Hosokawa, T. Synlett 2005, 631 .

138. Winkler, J. D.; Asselin, S. M.; Shepard, S.; Yuan, J. Org. Lett. 2004, 6, 3821.

139. Nadany, A. E.; Mckendrick, J. E. Synlett 2006, 2139.

140. Moriconi, E. J.; Crawford, W. C. J. Org. Chem. 1967, 33, 370.

141. Forró, E.; Fülöp, F. Tetrahedron: Asymmetry 2008, 19, 1005.

142. Chatterjee, A. K.; Choi, T-L.; Sanders, D. P.; Grubbs, R. H. J. Am. Chem. Soc. 2003, $125,11360$.

143. Hoveyda, A. H.; Lombardi, P. J.; O’Brien, R. V.; Zhugralin, A. R. J. Am. Chem. Soc. 2009, 131, 8378 .

144. Hoye, T. R.; Zhao, H. Org. Lett. 1999, 1, 1123.

145. Lin, Y. A.; Davis, B. G. Beilstein J. Org. Chem. 2010, 6, 1219.

146. Bouzbouz, S.; Cossy, J. Org. Lett. 2001, 3, 1451. 
ANNEX 